\title{
Exploring Thumb Input on Flexible Tablets
}

\author{
by
}

Md Moinuddin Sharif Riyadh

A thesis submitted to the Faculty of Graduate and Postdoctoral Affairs in partial fulfillment of the requirements for the degree of

Master of Applied Science

in

Human Computer Interaction

Carleton University

Ottawa, Ontario

(C) 2014

Md Moinuddin Sharif Riyadh 


\begin{abstract}
Flexible displays offer new interaction techniques such as bend gestures, but little work has been done to support touch input. Touch is the most common input method for handheld devices. We explore the potential of touch input using the thumb of the holding hand on flexible displays. We conducted two studies to evaluate tapping and swiping with thumb input, and compared the interactions between a flexible and a rigid tablet. Our result suggests that participants can perform tapping and swiping using thumb input similarly for both rigidities. This indicates that thumb input can offer the benefits of touch input in flexible displays. Our results also suggest that users prefer holding the display on the side or on the bottom corner over the bottom center. We present design guidelines to use thumb input on flexible devices, and provide three use case scenarios to visualize our design guidelines.
\end{abstract}




\section{Acknowledgements}

This work would not have been possible without the superior guidance and enormous support of my supervisor Dr. Audrey Girouard. I am grateful to her for believing in me when I contacted her from the other side of the world. I thank her for foreseeing my mistakes and always guiding me in the right way, for appreciating my efforts with inspiration, and for being extraordinarily patient with my silly mistakes. I thank her for enriching me with her vast knowledge and experience on HCI and technical writing skills, and for spending countless hours of her valuable time to read my numerous drafts and providing me with valuable feedbacks. I thank her for understanding all my problems, and dealing with them with cordial care. Most importantly, I want to thank her for being the amazing person she is, whom I can always respect at a personal level.

I thank Dr. Roel Vertegaal for providing the touch sensor for my experiments. I am grateful to Dr. Daniel Amyot for his sincere effort in teaching me software modeling, which helped me greatly during the prototyping process. I thank the HCI students and colleagues at Carleton University for assisting me during the user study sessions.

I thank my two roommates, Arifur Rahman and Raisul Alam, for making our place a second home for me. I am grateful to Mubashsharul Islam for always giving me the right support at the right time.

I want to thank my parents for being the best parents in the world and for always supporting me in every possible way. They are the reason behind all my success. I thank my two brothers, Nahid Helal and Rizvee Imran, for always feeling proud my achievements and for their constant inspiration. I thank, Nafees Rahman, for being the person I can always count on, and Noved Hoq, for being the family for me in Canada. 


\section{Table of Contents}

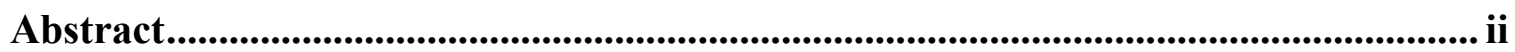

Acknowledgements ......................................................................................................ii

Table of Contents ........................................................................................................... iv

List of Tables ................................................................................................................. vii

List of Illustrations............................................................................................................... ix

1 Chapter: Introduction ....................................................................................................... 1

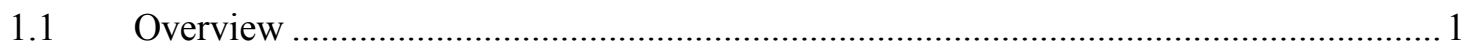

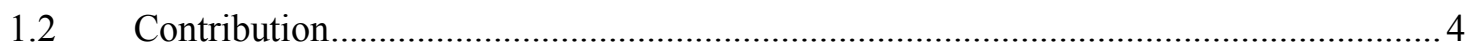

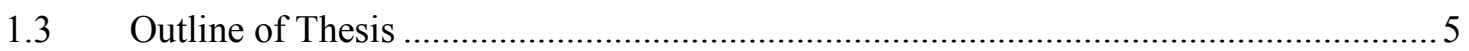

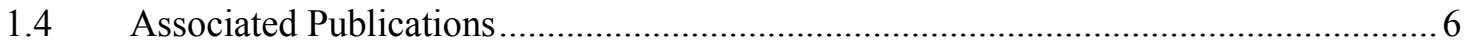

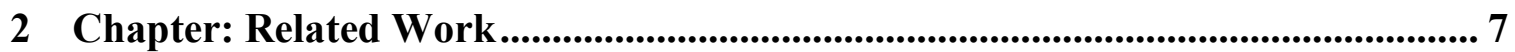

2.1 Thumb Input on Rigid Handheld Devices ................................................................

2.1.1 Identifying and Improving the User Performance of Thumb Input ..........................

2.1.2 Maximizing the Capability of Thumb Input ……………………………………... 10

2.2 Available Input Methods on Flexible Handheld Devices............................................ 12

2.2.1 Bend Gestures on Flexible Handheld Devices .......................................................... 12

2.2.2 Touch Input on Flexible Handheld Devices............................................................... 15

3 Chapter: Design Goals and Interaction Techniques ................................................ 17

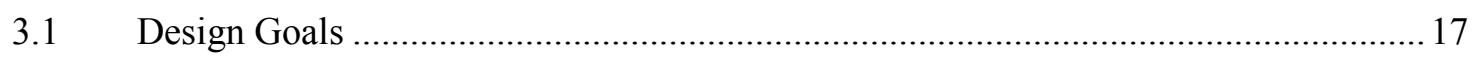

3.1.1 Bringing the Benefits of Touch Input in Flexible Displays ………………………... 17

3.1.2 Maximizing the Use of Holding Hand .................................................................. 18

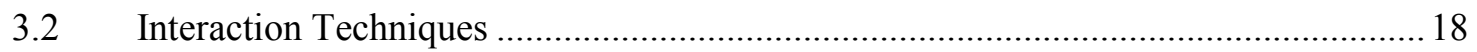

3.2.1 Hold Positions and Touch Regions ..................................................................... 18

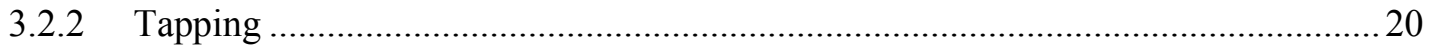




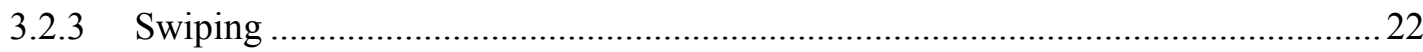

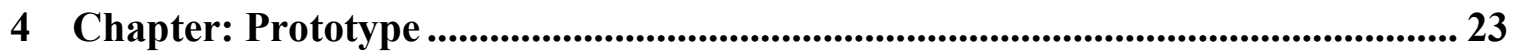

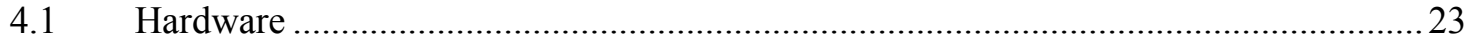

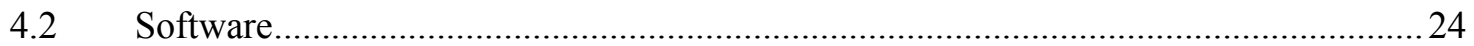

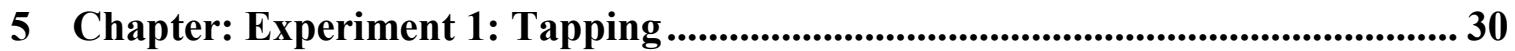

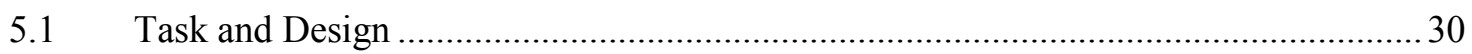

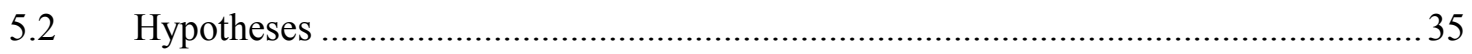

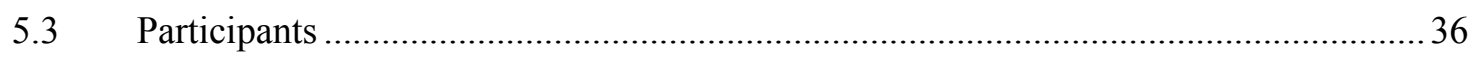

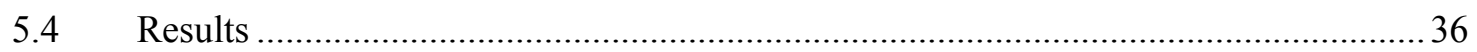

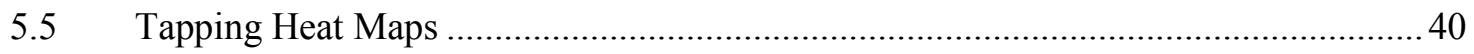

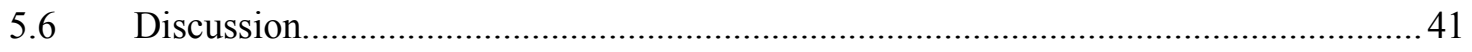

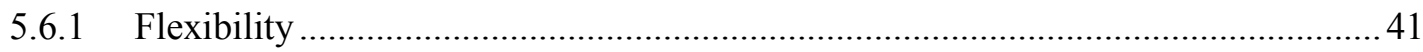

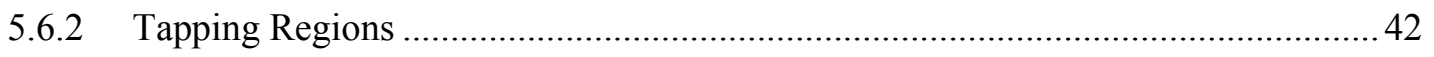

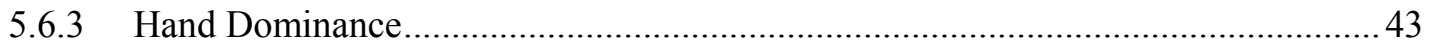

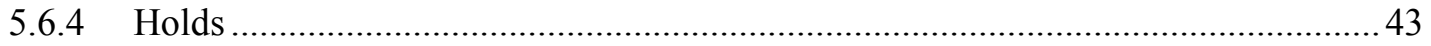

6 Chapter: Experiment 2: Swiping .......................................................................... 45

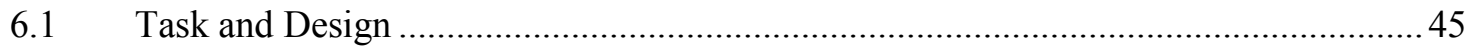

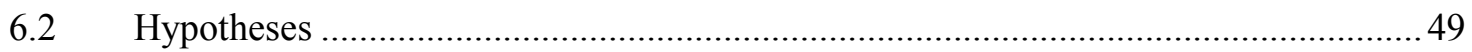

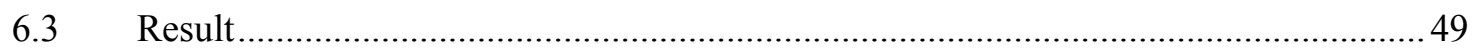

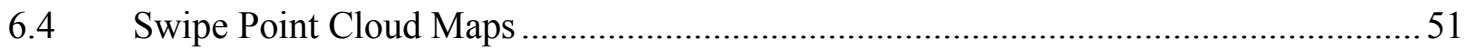

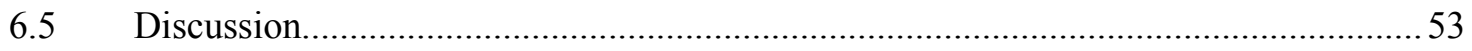

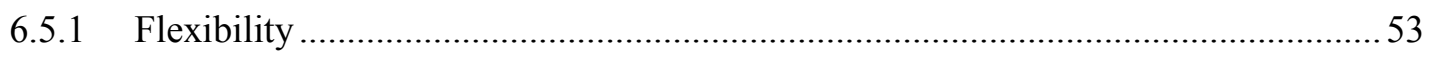

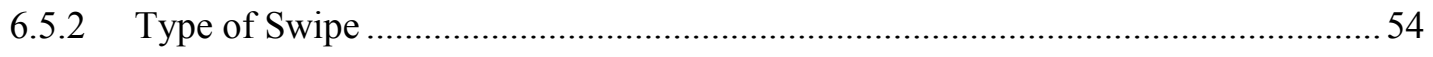

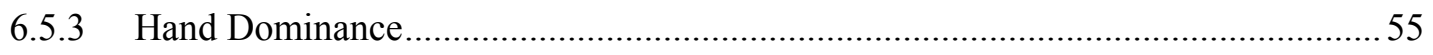

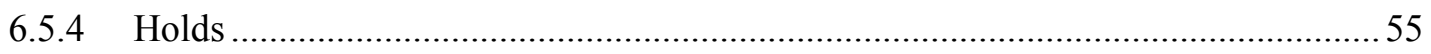


7 Chapter: Discussion ........................................................................................................ 57

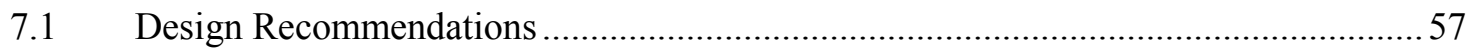

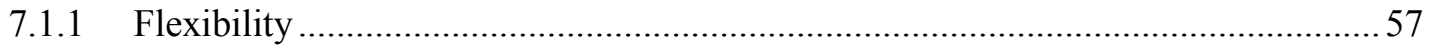

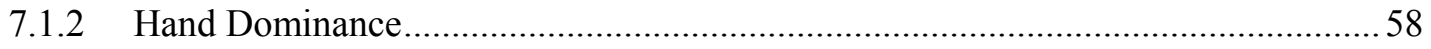

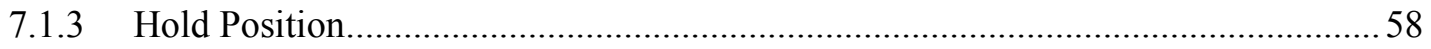

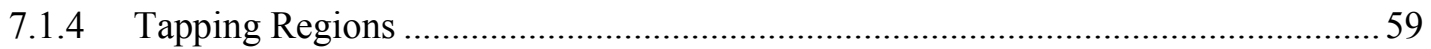

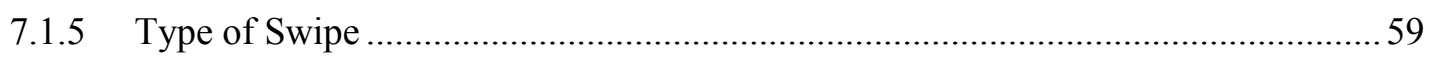

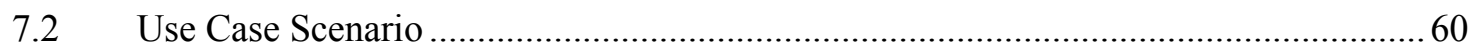

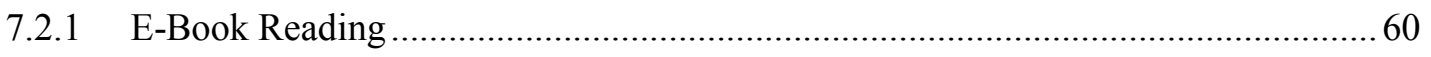

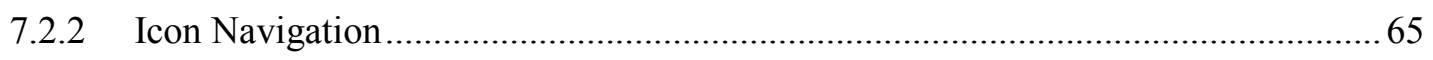

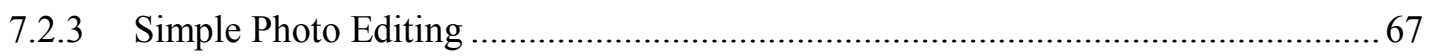

8 Chapter: Conclusion ....................................................................................................... 72

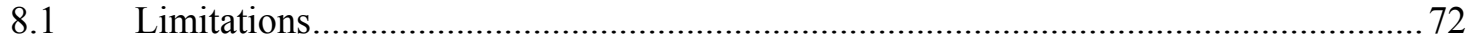

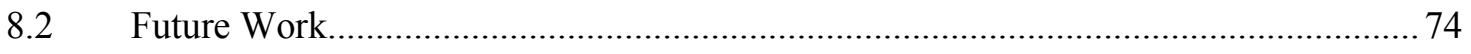

8.2.1 Absolute Direct Touch vs. Augmented Touch-pad................................................ 74

8.2.2 Bimanual Unimodal Interaction with Thumb Input ............................................ 74

8.2.3 Bimanual Multimodal Interaction with Touch and Bend..................................... 75

Appendices..................................................................................................................... 76

Appendix A Questionnaires ............................................................................................. 76

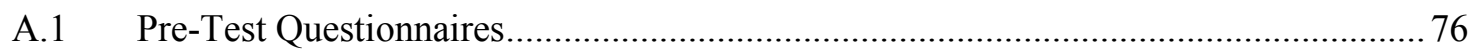

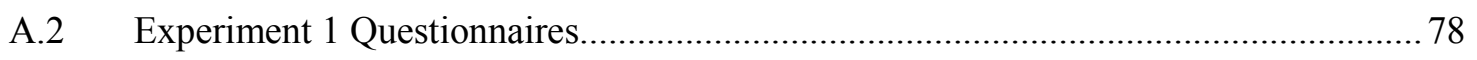

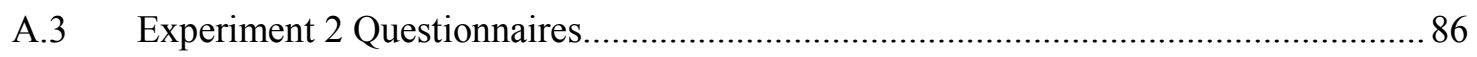

A.4 Overall Post-Test Questionnaires .................................................................... 90

Appendix B Regression Line Values ......................................................................... 93

B.1 Swipe Regression Line Values for the Flexible Prototype …....................................93

B.2 Swipe Regression Line Values for the Rigid Prototype .......................................... 94 


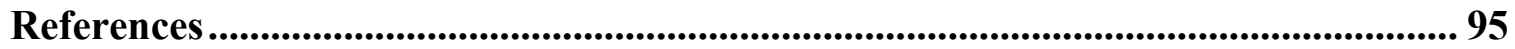




\section{List of Tables}

Table 1: Performance data (number of errors) for tapping interaction. Bold indicates

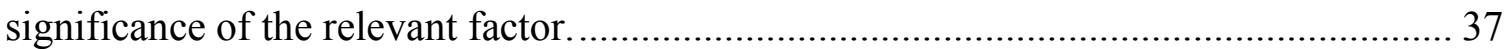

Table 2: Subjective data for tapping interaction. Bold indicates significance of the relevant factor.

Table 3. Subjective data for swipe interaction. Bold indicates significance. 


\section{List of Illustrations}

Figure 1: Tapping on the bottom corner hold position in a flexible tablet using thumb

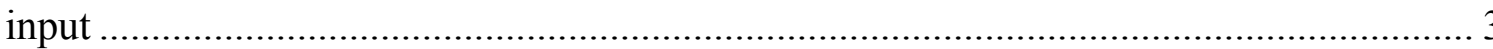

Figure 2: Three common hold positions: bottom corner, side center, and bottom center 19

Figure 3: Calculating the number of available tapping targets

21

Figure 4: Four types of swipe for left hand: (a) vertical, (b) horizontal, (c) diagonal, and

(d) radial.

22

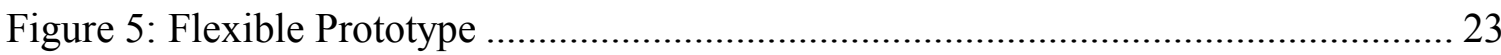

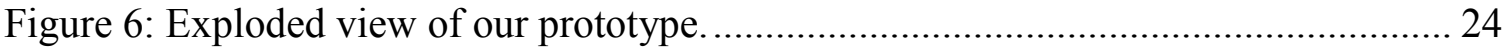

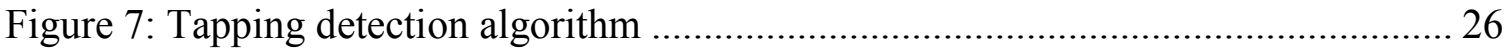

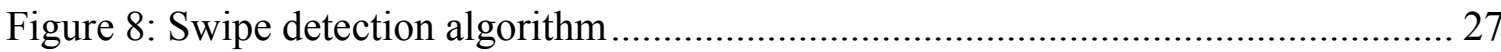

Figure 9: All printed touch regions. Three black shapes and two outlined shapes represent three and two tapping regions per hold. Shades of grey in each hold position represent the touch region for respective hold. 28

Figure 10: The application is informing user to start a task using the bottom corner hold position (left), tap the square shape in the bottom center (right-top), and perform the displayed swipe in the side center hold (right-bottom) ..................................................... 29

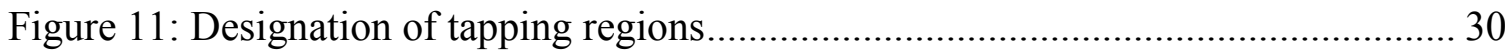

Figure 12: Tapping task using the flexible prototype ……….......................................... 32

Figure 13: Participant is performing the tapping task ................................................... 33

Figure 14: Mean tapping duration for flexibility, hold position and number of tapping regions per hold. Error bars represent standard deviation (SD). Asterisk indicates significance. 
Figure 15: Heat maps for two and three tapping regions per hold for three holds, both

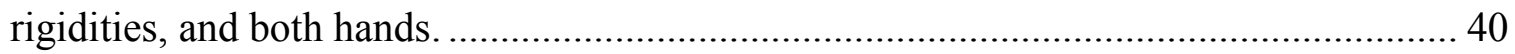

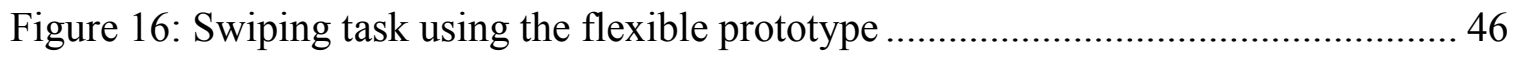
Figure 17: Mean swipe duration for rigidity, hold position, and hand. Error bars represent standard deviation. Asterisks indicate significance. ............................................... 50 Figure 18: Point cloud maps for four types of swipe for all holds, both rigidities and both hands. The regression line of each hold is indicated with a black line. 52 Figure 19: In an e-book reader, the user can use radial swipe with thumb input to navigate to the next or previous page (blue arrow). The user can also touch the tapping region of one of the two icons to either go to the home screen, or to access their library (white icon in gray zone). 62 Figure 20: The user can double tap in the adjacent area of any hold region to activate thumb input and visualize touch regions only for that particular hold. They can use radial swipe with thumb input in that region to navigate to the next or previous page (blue arrow). 64

Figure 21: In a home screen displaying icons of applications, the user can tap the 'folder' icon using their thumb to open the selected application (highlighted in red). 66 Figure 22: In the photo editing application, the user can increase the contrast of the image by swiping horizontally with the thumb (blue arrow). 68 Figure 23: In the photo editing application, users can crop an image with bimanual thumb input using horizontal swipe (blue arrows) 70 


\section{Chapter: Introduction}

\subsection{Overview}

Current trends in the consumption of computing devices is dominated by handheld rigid devices (i.e., smartphones, tablets) [10]. According to the International Data Corporation (IDC) report, tablet computing devices will have the highest percentage of growth taking up to $16.5 \%$ market share in 2017 [10]. While there is still no tablet with a flexible display for mass consumption, there is rapidly growing research on flexible displays with tablet form factor [4,17,30,34,35]. A tablet prototype with real flexible display called "PaperTab" was featured in the Consumer Electronics Show (CES) in 2013 [7]. PaperTab leveraged the benefits of electronics documents with the tangibility of paper documents [30].

There are many benefits of flexible displays over rigid displays. These displays are lightweight and thin, which allow users to hold them with one hand for extended period of time without much effort [35]. Flexible displays are potentially deformable like paper documents. Using this deformability, a novel interaction technique has been introduced in the context of flexible displays: bend gestures. Bend gestures are a feasible input method on flexible handheld devices $[16,17,29,30,34]$, and the deformability of flexible displays also facilitates mobility [35]. A standard sized tablet might be rolled up to fit inside a palm. Another benefit of flexible displays is that they consume less power than rigid displays $[16,35]$.

For handheld tablets, touch input is presently the most common interaction method [14]. While rigid tablet devices can take the full advantage of touch input, flexible displays currently provide limited support for touch interaction. Their main issue 
comes from the fact that they may not always provide adequate normal force to support touch input [4]. The distribution of normal force depends on the way they are held (e.g. on the corner, on the side; with one hand or two). Dijkstra et al. [4] showed that the holding hand provides the structural support required for pointing and dragging tasks using the other hand.

Extending Dijkstra et al.'s observations, we noticed that the holding hand may also provide sufficient normal force to support touch input in the adjacent region of that hold. As several studies revealed user preference for touch input using the thumb of the holding hand on rigid devices $[11,12,32]$, and the thumb's ability to interact with handheld rigid devices $[2,12,15,27,28,36]$, we were interested in testing the ability of the holding hand in a flexible device context. Specifically, we focused on touch input using the thumb of the holding hand on flexible tablets (Figure 1).

In addition to providing standalone thumb input, we believe it would be particularly useful when combined with bend interactions $[3,14,16]$. To bend a tabletsized display, users typically use both hands, one to hold the device and the other to bend it [16]. By using thumb input, the holding hand can add an additional dimension to bend gestures, bringing in the advantages of bimanual interactions to flexible displays $[18,24,32]$. 


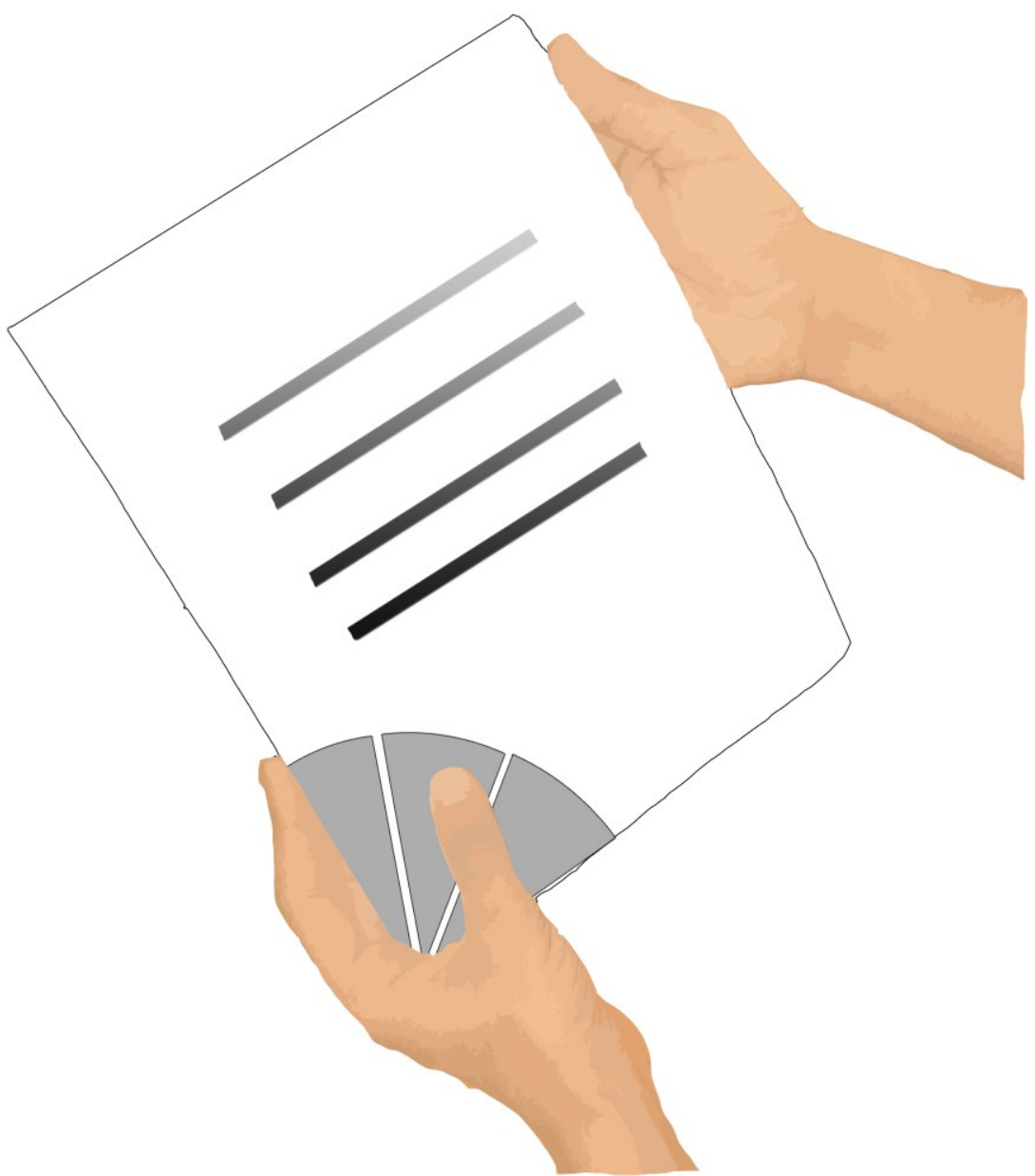

Figure 1: Tapping on the bottom corner hold position in a flexible tablet using thumb input

The two most common touch input methods used in present-day tablets are tapping and swiping. In this thesis, we investigated both, using the thumb of the holding hand. We conducted two studies with 21 young adult participants, one with tapping, and the other with swiping, to explore touch input using the thumb of the holding hand on flexible displays. We compared the performance and preference for tapping and swiping between a flexible and a rigid tablet prototype. We evaluated each interaction for both dominant and non-dominant hand.

Our primary research objective was to investigate the feasibility of touch input using the thumb of the holding hand on flexible tablets. We investigated how many 
tapping targets per hold users could handle comfortably using thumb input on flexible displays. Another goal of this thesis was to evaluate different types of swipe to identify preferences. In addition, we looked into the effects of hold position and hand dominance for thumb input on flexible handheld devices. We summarized our findings from both studies to provide design guidelines to integrate tapping and swiping interaction in tabletsized flexible displays using thumb input.

\subsection{Contribution}

We contribute to building the foundation of touch input using the thumb of the holding hand on flexible displays. As flexible displays do not provide enough support for touch input using the index finger [4], we were interested in exploring alternative ways to bring the benefits touch input to flexible displays. Extending prior studies on touch input on flexible displays $[3,4,14]$, we observed the potential of touch input using the thumb of the holding hand on flexible displays. We evaluated two basic touch interaction techniques, tapping and swiping, using thumb input on flexible displays.

Thumb input on rigid devices has been proved to be successful in maximizing the capability of the holding hand $[11,12,15,27,36]$. We believe the outcome of this thesis will pave the way to integrate those useful capabilities of thumb input on flexible handheld devices, eventually bringing the benefits of touch input to flexible displays. We also provide design recommendations to incorporate thumb input on flexible handheld devices. 


\subsection{Outline of Thesis}

This thesis is presented in eight chapters. The first chapter introduces the topic of thumb input on flexible displays and reveals the motivation and objective of this work. The following chapter reviews the prior works related to available input methods for flexible displays, and thumb input on rigid handheld devices.

In the third chapter, we discuss our design goals and interaction techniques for thumb input on flexible displays. The fourth chapter describes the hardware and software that construct the prototypes we used in both studies.

The fifth chapter provides the details about our first experiment, where users performed tapping interactions on a flexible and a rigid tablet prototype. This chapter includes the description of participants, tasks, procedure, hypothesis, results and discussion.

The sixth chapter describes our second experiment, where the participants were asked to perform swiping interactions on a flexible and a rigid tablet prototype. This chapter provides the details of the second experiment, including tasks, procedure, hypothesis, results and discussion.

In the seventh chapter, we provide design recommendations to incorporate thumb input on flexible tablets, along with three use case scenarios to visually present the potential usage of touch input using the thumb of the holding hand on flexible tablets.

The last chapter summarizes the main findings of this thesis, and discusses the limitations of this thesis and future works in this area. 


\subsection{Associated Publications}

Portions of this work have led to the following publication:

1. Riyadh, M. Exploring Tapping with Thumb Input for Flexible Tablets. In Proceedings of the SIGCHI Conference on Human Factors in Computing Systems, Student Research Competition, (2014). (6-page paper in ACM Digital Library, poster). 


\section{Chapter: Related Work}

To provide viable design guidelines to integrate thumb input on flexible tablets, we explored two groups of prior research: thumb input on rigid handheld devices, and the available input methods on flexible displays.

\subsection{Thumb Input on Rigid Handheld Devices}

We identified two high level objectives in the prior studies on thumb input on rigid handheld devices: some researchers focused on identifying and improving the user performance and accuracy of thumb input on rigid devices, while other researchers aimed at maximizing the capability of thumb input, mainly by offering customized user interfaces. We grouped the studies on thumb input on rigid handheld devices according to these two primary objectives.

\subsubsection{Identifying and Improving the User Performance of Thumb Input}

Interacting with a tablet using the thumb of the holding hand involves the carpometacarpal (CMC), metacarpophalangeal (MP), and interphalangeal (IP) joints [20]. While holding a tablet, they allow the contact point on the touchscreen to move along two degrees of freedom: abduction-adduction and flexion-extension. Though the free movement of the thumb is three dimensional, this motion trajectory of the thumb on the surface is two-dimensional [1]. Thumb's size and range of motion limit its reach on the handheld display surface while holding the display with the same hand. The following studies discuss these issues in detail to identify and increase user performance with thumb input on rigid handheld devices.

Goubier et al. [6] found that the mean abduction-adduction range of motion for thumb with guides was 68 degrees (range of $40^{\circ}$ to $93^{\circ}$ ), and the mean flexion-extension 
was 48 degrees (range of $30^{\circ}$ to $71^{\circ}$ ). They also measured the range of movement without guides: for flexion-extension it was 42 degrees (range of $30^{\circ}$ to $72^{\circ}$ ), and for abductionadduction, 53 degrees (range of $34^{\circ}$ to $69^{\circ}$ ). Odell et al. [21] correlated these values with the outcome of their study on thumb reach in tablet computers where they found that the range of thumb was longer in northeast-southwest direction, which is primarily generated by abduction-adduction, than the range of motion in northwest-southeast direction, which is generated by flexion-extension. Karlson et al. [13] also found that thumb movement along this axis to be easier and faster.

Otten et al. [22] conducted an study to determine thumb reach envelope for handheld devices. They recruited 90 participants in three groups(adolescent females, young mothers, and young fathers) for their study and found that an area between 45.6 $\mathrm{mm}$ and $49.4 \mathrm{~mm}$ from the bottom and between $25.4 \mathrm{~mm}$ and $35.5 \mathrm{~mm}$ from the right was reached by more than $90 \%$ of the participants. They mentioned that these movements map closely to abduction-adduction and flexion-extension of thumb. They also suggested that the age was a factor in thumb reach, and they reported that adolescent females had larger thumb reach envelope than the other two adult groups.

Oulasvirta et al. [23] found that in landscape orientation using the side-bottom grip in a tablet, their participants could reach a region with a width of $57.6 \mathrm{~mm}$. Bergstrom-Lehtovirta et al. [1] proposed a mathematical model to calculate the functional area of the thumb of the holding hand on a touchscreen surface and they applied their model to Oulasvirta et al.'s data [23], and found that the functional area of thumb crossed the bottom edge of the device at $5.79 \mathrm{~cm}$ in side-bottom grip. Their model also suggests 
that the thumb can reach both the side and bottom edge of the tablet using the sidebottom grip.

Parhi et al. [25] conducted a two-phase study to determine the optimal target size for one-handed touch input using the thumb on a handheld device. Their findings suggest that the target size for thumb use should be at least $9.2 \mathrm{~mm}$ for single target tasks (e.g., selecting a button or checkbox) and $9.6 \mathrm{~mm}$ for multi-target tasks (e.g., text or numerical entry). The researchers also showed that in a $3 \times 3$ region matrix, the center target is the most preferred one. Karlson et al. [13] and Henze et al. [8] supported the preference for the center target for a larger number of targets.

In a similar study, Park et al. [26] evaluated three different target sizes (4 mm, 7 $\mathrm{mm}, 10 \mathrm{~mm}$ ) and twenty five locations to investigate their effects on single-handed usage of touch-screen mobile device with the thumb. They found performance and subjective satisfaction to be correlated with target size: smaller target sizes led to lower performance and satisfaction levels. Their results also suggest that the locations that are too distant for our thumb to reach, and the locations that require an angular movement by the thumb generally produced lower user performance and preference.

Karlson et al. [11] investigated one-handed thumb use for PDAs and cell phones. They evaluated swipes in four sets of directions: vertical, horizontal, diagonal and radial swipes. Their findings suggested that the users were comfortable and efficient performing horizontal, vertical and radial swipes while they struggled to perform the diagonal ones.

Wagner et al. [32] introduced BiTouch, a design space that addresses bimanual interactions on rigid handheld devices. The authors identified five common holds for tablets: bottom center, bottom (right) corner, side (left) center, top side towards the right 
corner, right side towards the top corner. Three out of these five holds use thumb for touch input: bottom center, bottom corner and side center. Based on BiTouch, the researchers developed BiPad toolkit to help developers add bimanual interaction to multitouch tablets. Their study showed that the thumb outperformed fingers in both orientations for taps, and that gestures using thumb were significantly faster than with fingers in portrait orientation.

These studies provide guidelines for efficient thumb input on rigid handheld devices. They evaluated tapping and swiping interactions with thumb input to determine and improve user performance for thumb input on rigid handheld devices.

\subsubsection{Maximizing the Capability of Thumb Input}

Given the thumb's limited operational range [2] while holding the device, researchers have designed interaction techniques to investigate increasing the capability of thumb input. We briefly discuss these studies in this section.

ThumbSpace is a software-based interaction technique that helps users reach the far targets of the screen using thumb input [12]. The authors used a 'radar-view' of the entire interface to augment a touch-pad within the reach of thumb. The users can use that augmented touch-pad to access all the locations of the interface. The authors suggested that this superimposed touch-pad cannot replace direct touch on standard touch screen devices, but it can improve the touch interaction on handheld devices when used as an additional input dimension with direct touch. Instead of tracking $\mathrm{x}$, y position of touch points for interaction, Boring et al. [2] studied a different input dimension: variation in thumb's contact size during thumb interaction. They used small contact size of thumb for panning. Increasing the contact size switches to zooming operation, and further increment 
in contact size causes a faster zoom. This input dimension is less fatiguing than a slider, Tilt-to-Zoom [9] and CycloStar [19] .

TapTap and MagStick are two thumb interaction techniques that address common issues raised by the target selection using the thumb on small displays: screen accessibility, visual occlusion, and accuracy [27]. TapTap provides a magnified view of the area of interest with a single tap, and a second tap that is used to select the desired target from the magnified view. MagStick provides a 'magnetized' cursor, controlled by a telescopic stick, which appears on the screen when the user presses the screen and drags the thumb. Both techniques were designed to leverage the capability of thumb input on rigid handheld devices. The authors compared them with direct touch, ThumbSpace [12] and other interaction techniques for thumb input on small handheld displays. TapTap had the lowest error rate of all techniques and it was the second fastest technique after direct touch. MagStick was the fastest technique after direct touch and TapTap, and it had the lowest error rates after TapTap.

Kim et al. [15] presented two triggering techniques (Edge and Large Touch) and two selection techniques (Sliding Screen and Extendible Cursor) to increase the reachability of the thumb on large-screen mobile devices in both portrait and landscape orientation. Both selection techniques could be activated by either triggering techniques. In Edge Touch, users performed a swipe from the edge of the screen to activate the selection mode, whereas in Large Touch, users touched anywhere on the display with the thumb-pad to trigger selection mode. After a selection is triggered, in Sliding Screen, users simply slid the screen to make the farther targets reachable by the thumb. 
The Extendible Cursor works as MagStick [27]. However, it uses an invisible stick, but displays a cursor which appears once this selection mode is activated. The authors found all of their proposed interaction techniques to have significant advantages for distant targets, especially in landscape orientation. Overall, they found Edge triggering with Extendible Cursor to be the best combination. Yu et al. [36] conducted a similar study with two target acquisition techniques (CornerSpace and BezelSpace) using the thumb of the holding hand in a large screen mobile device. Their results suggest that using their interaction techniques, thumb input can be effectively used to reach the distant targets of a large screen smartphone.

These studies show how thumb input can effectively improve the user experience on rigid devices, and allow users to do more with their holding hand. To the best of our knowledge, these interaction methods for thumb input have not been yet evaluated with flexible devices.

\subsection{Available Input Methods on Flexible Handheld Devices}

The majority of studies on interaction techniques for flexible displays have focused solely on bend gestures. Bend gestures are a new interaction technique offered by the flexibility of the deformable displays. A few studies also evaluated touch input on flexible displays. In the following two subsections, we discuss bend gestures and touch input on flexible handheld devices.

\subsubsection{Bend Gestures on Flexible Handheld Devices}

Most of the studies on flexible displays have evaluated bend gestures for different tasks to provide design guidelines to integrate this novel input method on flexible 
displays. In this section, we briefly discuss about these studies on bend gestures for flexible handheld devices.

Schwesig et al. [29] introduced Gummi, a bendable handheld computer concept, and a set of interaction techniques that leverage bending as an input technique to manipulate digital content. They emphasized embodied interfaces that take advantage of bending, with two dimensional position control. The authors grouped closely-related opposite tasks and mapped them into two major bending techniques: up and down. They evaluated bend gestures for both complex tasks (e.g.: typing) and simple navigational tasks. Their result suggests that Gummi interaction techniques are feasible, effective and enjoyable. However, the authors mentioned that their proposed bend interaction techniques cannot replicate the full range of functionalities offered by WIMP-based (Windows, Icons, Menus, Pointer) user interfaces.

PaperPhone is a smartphone-inspired flexible E-Ink prototype which has a rigid bezel on the left side and three locations for bend gesture input on the right side [16]. The authors' goal was to build a simple interaction language for bend gestures. They evaluated the effectiveness of various bend gestures in executing a set of tasks with a flexible display. Their results showed that the users preferred bend gestures that were conceptually simpler, e.g., along one axis, and less physically demanding. Their results also suggest that bend gestures form a useful addition to interaction modalities of future flexible computer.

Lee et al. [17] took a user-centered approach to better understand the deformation-based interactions at different flexibility levels. The researchers used three types of A4-sized prototype of different materials: paper, elastic clothes and plastic. They 
categorized seven types of deformation: bending, twisting, folding, rolling, crumpling, tearing and stretching. The three types of prototypes used in this study support these seven categories of deformation all together. Participants were spontaneous and took shorter time to perform those gestures that were metaphors of common daily actions. They also showed high preference for closely-related opposite gestures. They preferred horizontal orientation though the devices were initially placed in a diagonal position. From the analysis, the researchers also discovered the importance of gestural synonyms. As opposed to their initial assumption, the authors discovered that the higher device flexibility actually produced higher gesture agreement.

Wightman et al. [35] presented design guidelines for flexible e-book readers by comparing various interaction styles using their prototype: BendFlip. The researchers focused on one of the most common tasks of e-book reading experience: single page navigation. They chose five types of input techniques for evaluation using their prototype: capacitive touch sensors, pressure sensor buttons on the front and back of the display, pressure sensors with one-handed interactions and three different variations of bend gestures on the side of the display. Their analysis suggests that the bend gesture is a better alternative to button for flexible e-book readers. They also mentioned avoiding capacitive input and minimal force button on flexible devices as they increase spurious interactions.

Results of such flexible displays studies show that the bend gestures are a feasible and effective input method for deformable handheld computing devices. However, none of these studies suggest bend gestures as a replacement for touch input. 


\subsubsection{Touch Input on Flexible Handheld Devices}

While the majority of studies on flexible displays were dominated by the

investigation of bend gestures, some studies evaluated touch input on flexible displays. We introduce these studies in this section.

Dijkstra et al. [4] evaluated the effects of structural holds on pointing and dragging performance with flexible displays by using three common holds: bottom center, bottom corner, and side center. These holds provide normal force to their adjacent areas in the display surface to support touch input. These adjacent areas possess higher rigidity than the other areas of the display. Their results indicated that the performance in pointing and dragging tasks is, on average, $12 \%$ better on the areas with higher rigidity than on the more flexible zones of the display. However, they did not evaluate thumb input on flexible displays. Instead, they concluded that the presence of thumb in 'holdzones' interfered with touch sensing in the rest of the device surface. They suggested that using a bezel on flexible screens may solve this problem.

Kildal et al. [14] investigated the potential of combining deformation and touch simultaneously on a flexible handheld device. They measured pragmatic and hedonic aspects of interaction with three different interaction designs applied to a photo manipulation application: two hybrid designs combining deformation and touch, and one design using only multitouch. Results show that all three input techniques provided equivalent performance. The researchers recommended using front-touch (i.e., touch input on the front surface of the device) in conjunction with deformation instead of backtouch (i.e., touch input on the back of the device). Their findings revealed that the fronttouch was popular as the users could use their thumb instead of their index finger. 
In FlexView, Burstyn et al. [3] proposed flexible scrolling methods, including utilizing touch input using the thumb for vertical and horizontal scrolling. Their findings suggest that the thumb has a more comfortable range of vertical motion afforded by gripping the side of the display. They also indicated that by augmenting touch with bend in parallel improves user experience in flexible devices.

These studies briefly mention thumb input but none of them investigated it in detail. This indicates the need to explore thumb input further in a detailed study. In this thesis, we aim to combine the results from thumb studies on rigid devices with general flexible touch evaluations. 


\section{Chapter: Design Goals and Interaction Techniques}

\subsection{Design Goals}

Touch interaction has become the most common interaction method for handheld devices [14]. The introduction of flexible display technology has brought new focus on alternative input methods for those devices, specifically, bend gestures [16,17,29,35]. While many researchers have found bend gestures to be a feasible and effective input method for flexible displays, they have also expected bend gestures to coexist with touch input rather than being a stand-alone input method $[3,14,29]$.

However, the physical properties of flexible displays impose limitations on the use of touch input [4]. Dijkstra et al. [4] showed that the normal force created by the fingers of the gripping hand creates touch-friendly areas in certain regions of the surface of a handheld flexible display. When a user holds a flexible display, the regions accessible with the thumb of the holding hand may also possess enough normal force to support touch input generated by the other fingers of the same hand. Although thumb input is a common touch interaction, and has been proved to be useful in rigid handheld devices, it has not been investigated in detail in the domain of flexible displays $[3,14]$. In this thesis, we explored thumb input on flexible handheld devices with the following two design goals:

\subsubsection{Bringing the Benefits of Touch Input in Flexible Displays}

While touch input using the index finger struggles to offer an effective solution to bring the benefits of touch interactions on flexible displays [4], we are interested in evaluating the potential of thumb input on flexible displays. Thumb input improves the user experience in rigid handheld devices $[11,12,15,27,36]$. We believe that by using 
thumb input, users can enjoy the benefits of touch input in flexible displays, which can eventually improve the user experience in flexible handheld devices.

\subsubsection{Maximizing the Use of Holding Hand}

In flexible displays, the users often hold the device with one-hand, and interact with it using the other hand to perform bend gestures [16], as illustrated in Figure 1. In such case, the holding hand does not significantly contribute to the interaction, except for holding the device. We are interested in maximizing the use of the holding hand by using thumb input on flexible displays. We expect that this will also offer the opportunity to integrate bend and touch in parallel on flexible displays for bimanual interactions.

\subsection{Interaction Techniques}

\subsubsection{Hold Positions and Touch Regions}

Wagner et al. [32] identified five common hold positions when using a rigid tablet, with three holds incorporating touch input using the thumb of the holding hand: the bottom corner, bottom center and side center. Dijkstra et al. [4] found in parallel the same three hold positions with tablet-sized deformable displays. In this thesis, we investigated both tapping and swiping interactions using these three hold positions in portrait mode. We identify those three in Figure 2. 

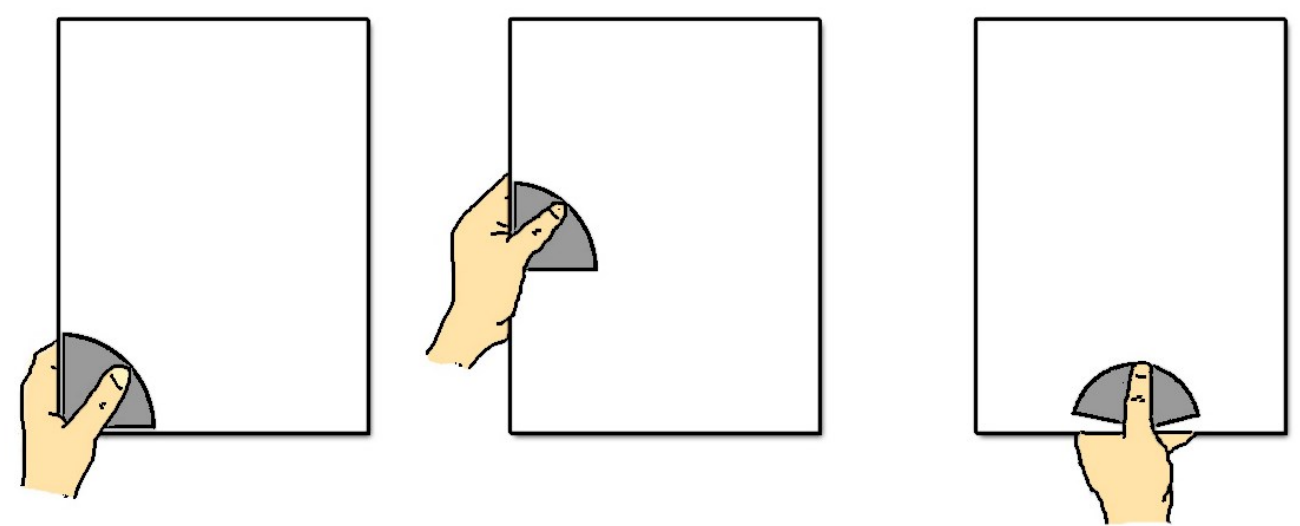

Figure 2: Three common hold positions: bottom corner, side center, and bottom center

We wanted to accommodate thumb sizes for the majority of users in the adjacent touch region of each hold for thumb input. This led us to use a larger touch area than the average thumb reach envelope for handheld devices [21,22]. To estimate the touch areas, we started with the basic measures outlined in the prior work, and we went through an iterative design process with 8 pilot participants. We observed that, given the pattern of the side center and bottom corner hold positions, users had a slightly more reach along the vertical axis than the horizontal axis for that two holds, which was also observed in prior work $[1,3]$. We reflected this finding in our touch region design and defined the bottom corner touch region to reach the bottom edge at $6 \mathrm{~cm}$ and side edge at $6.5 \mathrm{~cm}$ (both are measured from the bottom corner of the device). We defined the same area for the side center hold as the holding pattern for side center hold was similar to the bottom corner hold (Figure 2).

In case of the bottom center hold, we noticed that users had more difficulty in the horizontal thumb movement. As a result, we used a slightly smaller width $(5.5 \mathrm{~cm})$ for this touch region. The height of the touch area for this hold was similar to the other bottom corner and the side center holds. However, though the touch area for bottom 
center hold was $0.5 \mathrm{~cm}$ less than the other two holds, active touch areas for all hold regions were eventually similar as there was a bezel of $0.5 \mathrm{~cm}$ on both sides of the device, which was included in the measurement for adjacent touch regions of side center and bottom corner holds. This similarity among the touch regions for all hold positions reduced the complexity in our analysis as we compared these three holds with each other for both tapping and swiping interaction. We further expand on these estimated regions in the calculation for tapping regions (Figure 3).

\subsubsection{Tapping}

Tapping is the most common touch interaction technique on rigid handheld devices. We chose the single tap, the simplest tapping interactions used in rigid devices, to verify its compatibility on flexible devices using thumb input. We designed our experiment on tapping interactions to find how many tapping targets per hold participants can handle with ease. We created large tapping targets, i.e. tapping regions to accommodate different thumb length.

Users like to reach targets approximately $1 \mathrm{~cm}$ wide [25], with the thumb naturally extended [28]. Unlike finger touch, touch input using the thumb utilizes the thumb pad instead of the tip, especially in the case of touch using the thumb of the holding hand, as the thumb is parallel to the surface. This decreases thumb's reach on the display surface of handheld devices, and these factors restrict the number of available tapping targets. We calculated the number of available tapping targets per hold (Figure 3).

The numbers below represent the average values for 50 percentile average man and 50 percentile average woman: 
Thumb length $=5.65 \mathrm{~cm}[31]$

Thumb width $=2.1 \mathrm{~cm}[31]$

Thumb tip to pad distance $=1.15 \mathrm{~cm}[31]$

$R=$ thumb length - distance between tip and pad $=5.65-1.15=4.5 \mathrm{~cm}$ (Figure 3)

$S=R^{*} \theta=4.5^{*}\left(\left(90^{\circ} * \pi\right) / 180^{\circ}\right)=7.1 \mathrm{~cm}$

$X=$ thumb width $=2.1 \mathrm{~cm}$

$S / X=7.1 / 2.1=3.3$

Available targets $=3$

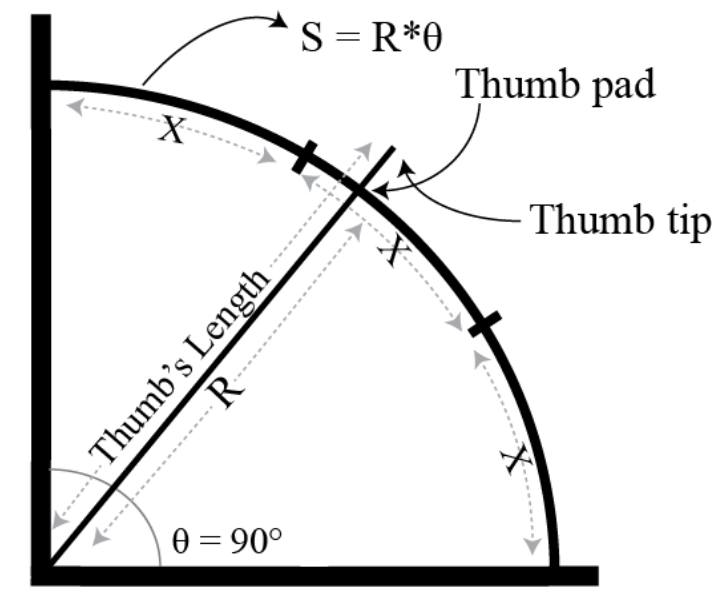

Figure 3: Calculating the number of available tapping targets.

This estimate suggests that each hold can accommodate a maximum of three nonoverlapping tapping targets for thumb input, for the average adult user. We conducted an informal study with five participants to verify these results, and tested if the participants could distinguish more than three tapping regions per hold with ease: three participants had difficulty distinguishing more than three, and two mentioned not being comfortable with more than two. This outcome identified the need to further investigate how 
participant performance and preference may vary for two and three tapping regions per hold.

\subsubsection{Swiping}

Swipe is a widely used touch interaction technique on rigid tablets. Common tasks like scrolling a page, browsing a photo gallery, navigating a map involve swipe interactions. We believe that the use of swipe on flexible handheld devices would enable participants to enjoy some of the advantages of touch interaction. Hence, we explored the potential of swipe interaction on flexible displays using the thumb of the holding hand.

Karlson et al. [11] studied four types of swipes with thumb input on a rigid device: horizontal, vertical, diagonal and radial swipes (Figure 4). We considered these four types of swipes and investigated their compatibility on a tablet-sized flexible device. As Figure 3 suggests, all the swipes are bidirectional, e.g., a vertical swipe has two directions: up and down. We tested both directions for all four types of swipe.

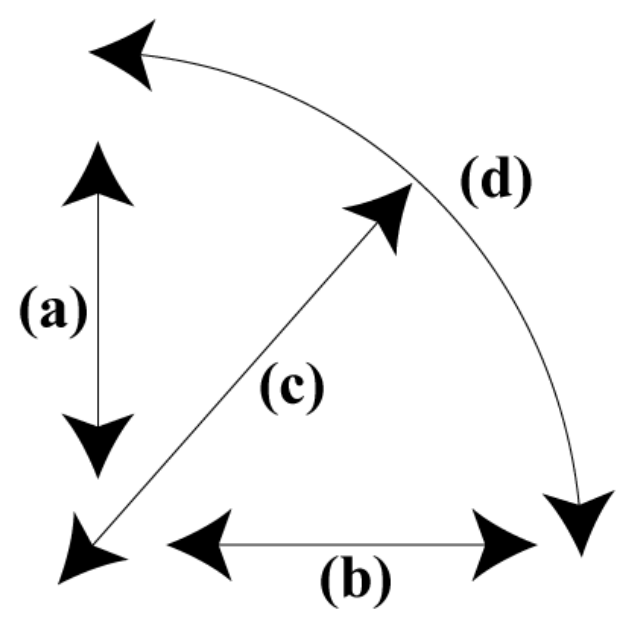

Figure 4: Four types of swipe for left hand: (a) vertical, (b) horizontal, (c) diagonal, and (d) radial. 


\section{Chapter: Prototype}

\subsection{Hardware}

Our prototype was composed of 6 layers, with a total thickness of $3.25 \mathrm{~mm}$, and an extra layer of Plexi Glass (2 mm) for the rigid condition (Figure 5, Figure 6). We used a 10.06" x 7.17" flexible and transparent Zytronic touch sensor to detect touch input [37]. We used two Styrofoam layers beneath the touch sensor to provide insulation, helping participants avoid accidental activation of touch from the back of the sensor.

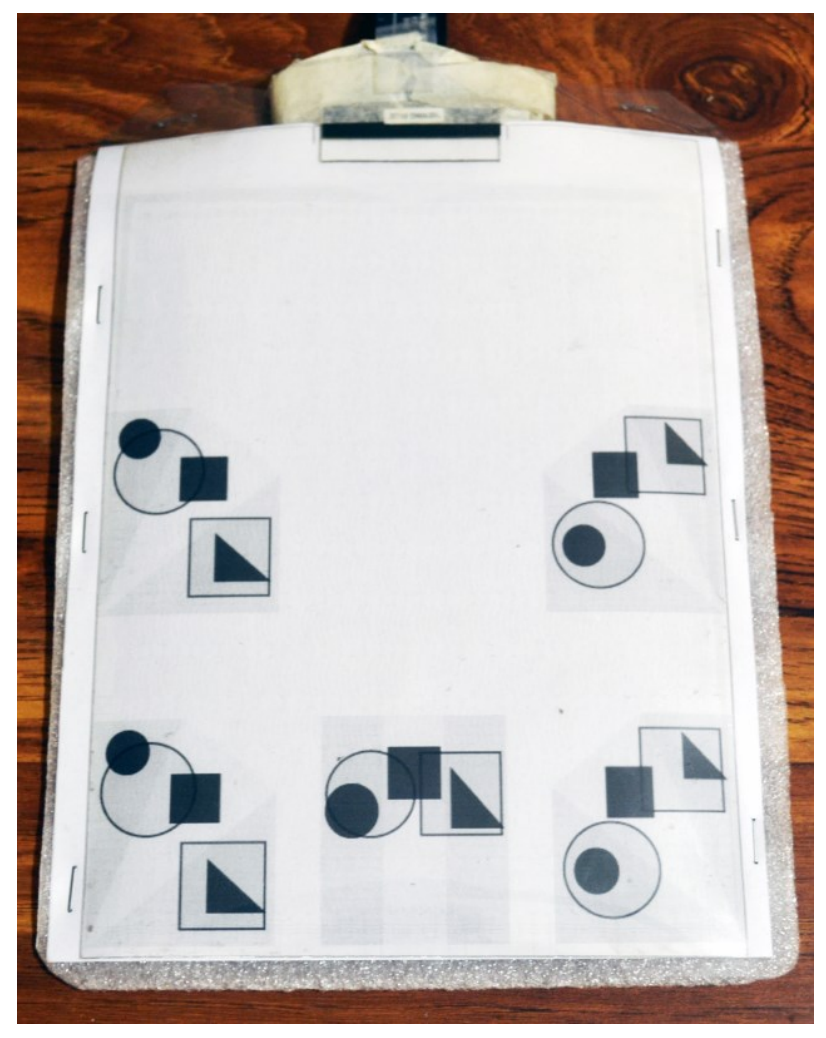

Figure 5: Flexible Prototype

Two thin transparent plastic sheets wrapped the prototype from the front and back. These two plastic sheets were used to hold the other layers of the prototype altogether. They also provided a smooth surface both in the back and the front of the 
device, which contributed to a comfortable grip of the device as well as smooth transition while switching hold position.

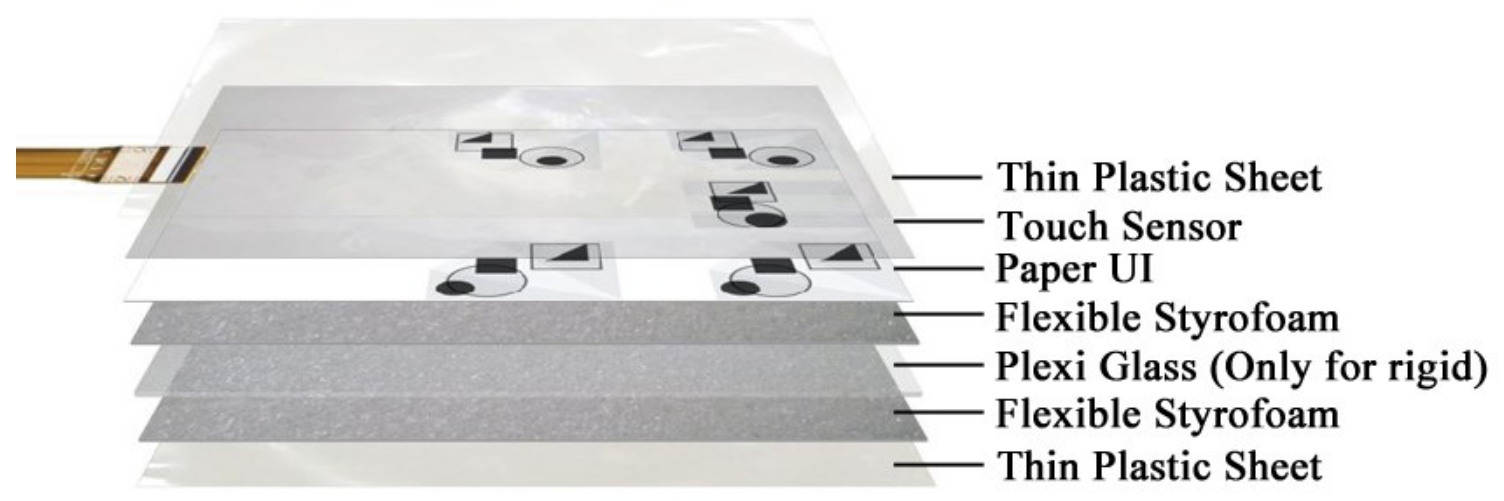

Figure 6: Exploded view of our prototype.

A paper sheet was placed immediately under the transparent sensor to indicate the fixed tapping regions and touch regions (Figure 9). We used filled black shapes for three tapping regions per hold and outlined shapes for two tapping regions. The shapes were placed in the touch regions in a consistent order for both hands (i.e., the sequence from left to right: circle, rectangle, and triangle). The gray areas represent the touch region for each hold region. Any interaction, tapping or swiping, must be performed within these gray regions for respective holds. We confirmed the overlapping tapping regions with our five pilot participants.

\subsection{Software}

The prototype (i.e., the touch sensor) was connected to a laptop computer via USB. A WPF (Windows Presentation Foundation) application was developed using .NET Framework (version 4) with C\#. The application detects tapping and swiping interactions and displays relevant information on the screen. The fixed touch regions were defined in the application to detect tapping and swiping for three hold positions, matching the paper 
sheet on the prototype. The tapping detection algorithm uses $x-y$ coordinates, whereas the swiping detection algorithm uses the length of the interaction, between 'touch down' and 'touch up' events, in addition to x-y coordinates. We used the 'length' parameter to define a minimum displacement of thumb between 'touch down' and 'touch up', in order to identify a swipe.

To identify a correct interaction for both tapping and swiping, the detection algorithm collected user input from the touch sensor and checked it with all the relevant conditions for each interaction and controlled the flow of the application. Whenever a touch event occurred (when users touched the sensor), the algorithm first checked the type of task (tapping or swiping), then matched the input value for the pre-set task's conditions. When the conditions were met, the interaction was identified as a correct interaction. Figure 7 and Figure 8 are flow-charts of tapping and swiping detection algorithm respectively. 


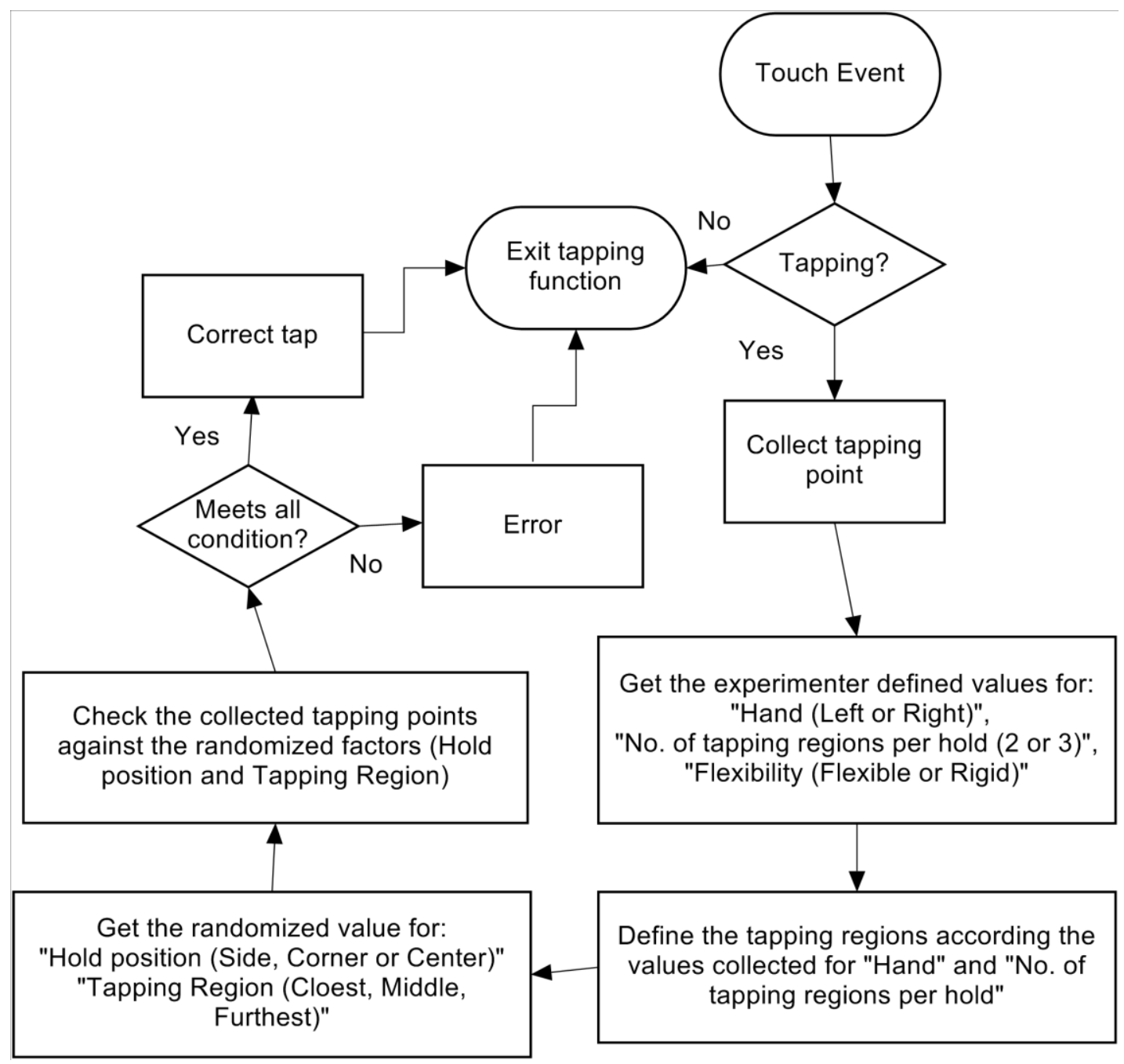

Figure 7: Tapping detection algorithm 


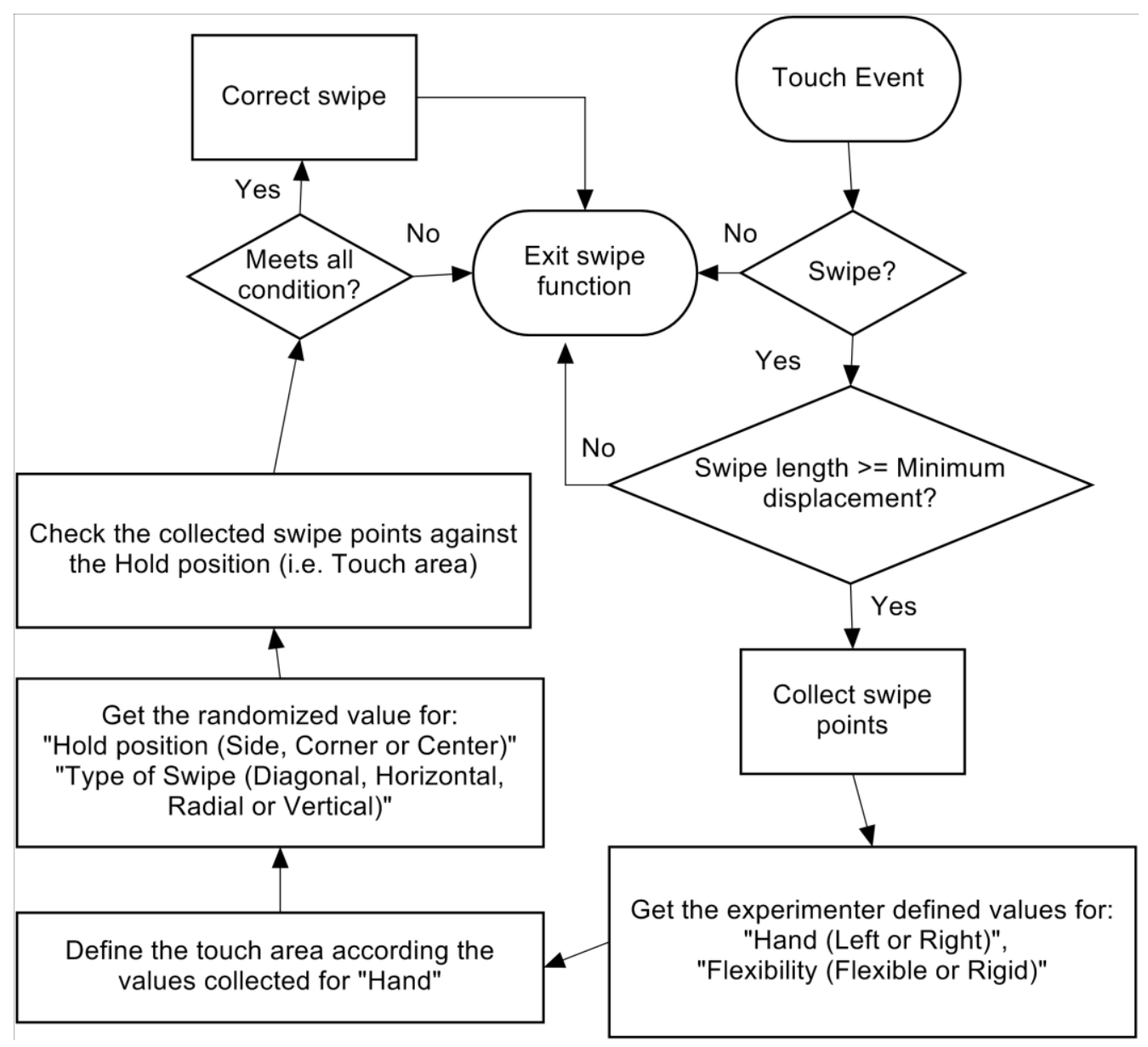

Figure 8: Swipe detection algorithm 


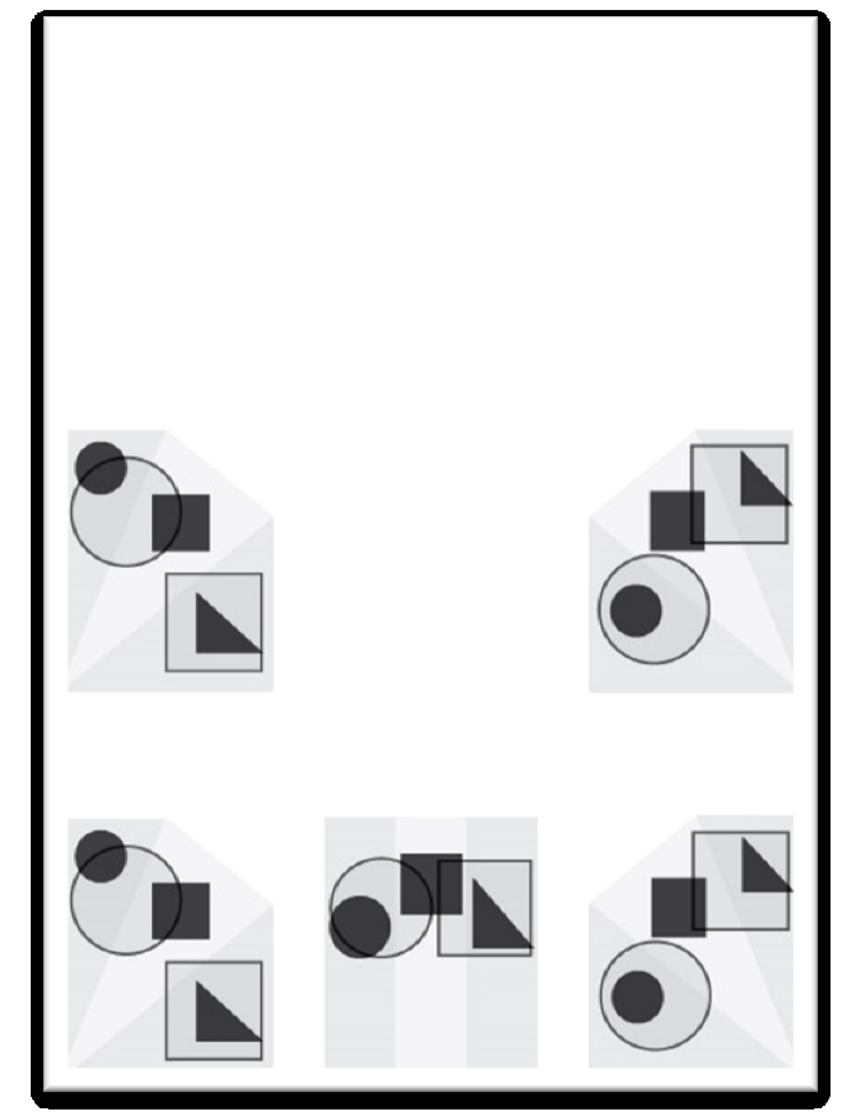

Figure 9: All printed touch regions. Three black shapes and two outlined shapes represent three and two tapping regions per hold. Shades of grey in each hold position represent the touch region for respective hold.

We used a pico-projector to create a dynamic display on the prototype. Using the pico projector, the application shows the current task to the user, by displaying tapping targets (square, triangle or circle) or type of swipe (horizontal, radial, diagonal, or vertical) with direction. The application provides visual information about which hold position a user needs to use, in a yellow box on the top of the prototype surface. Finally, the display also visually informs user about when to start a task and when the task is complete (Figure 10). 


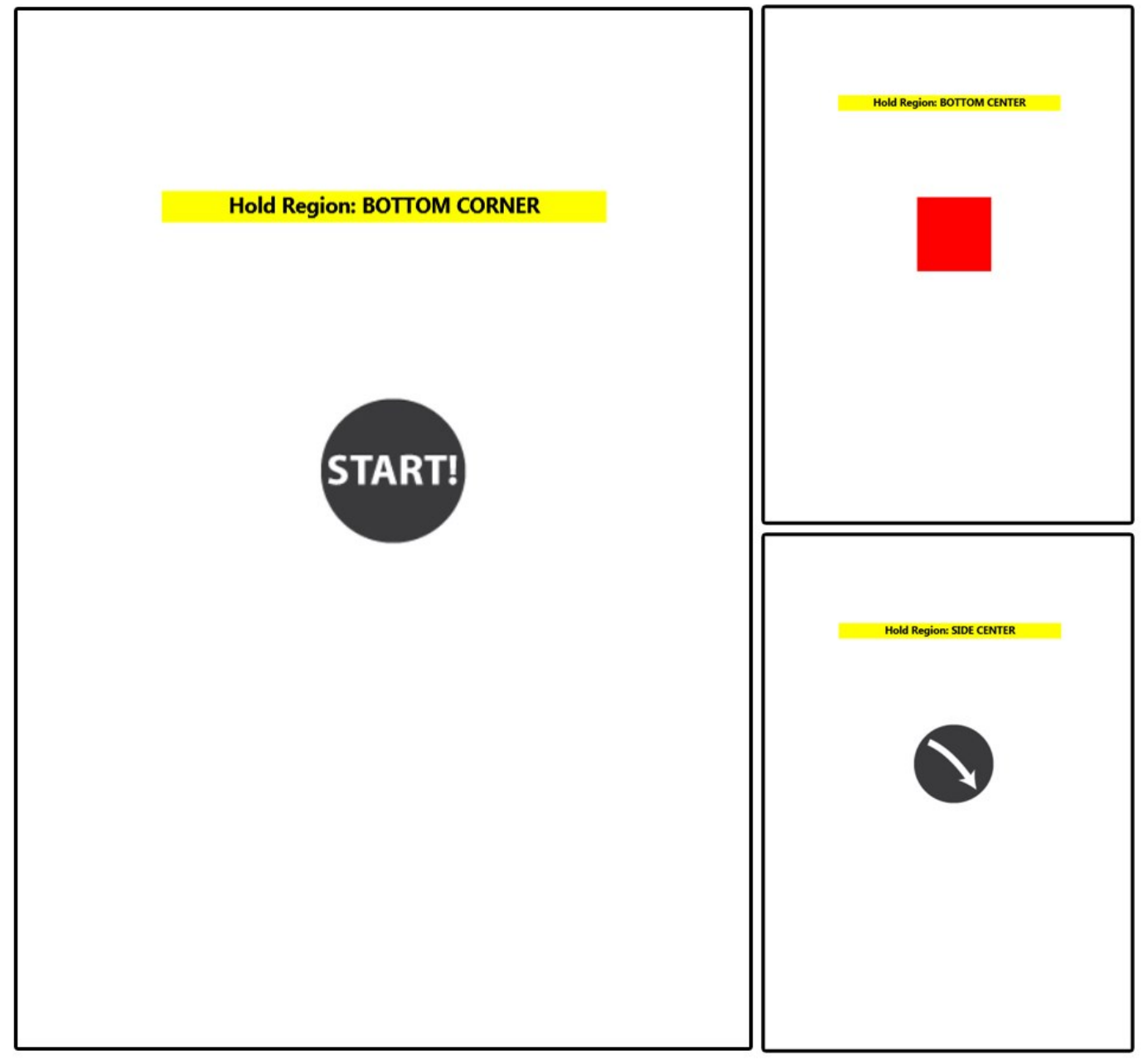

Figure 10: The application is informing user to start a task using the bottom corner hold position (left), tap the square shape in the bottom center (right-top), and perform the displayed swipe in the side center hold (right-bottom). 


\section{Chapter: Experiment 1: Tapping}

We designed our first study to evaluate the tapping abilities of the thumb on a flexible tablet, by comparing a flexible and a rigid prototype. We evaluated tapping interaction in three common holds, with both the dominant and the non-dominant hand, as participant preference and performance for tapping interactions may vary between two hands.

Finally, we assessed two sets of tapping regions ( 2 and 3 tapping regions).

\subsection{Task and Design}

We designed our first experiment with six factors: flexibility (rigid or flexible), number of tapping regions per hold (two or three), hold positions (bottom corner, side center, and bottom center), hand (dominant or non-dominant), active regions for two tapping regions per hold (closest region to the index finger, furthest to the index finger), and active regions for three tapping regions per hold (closet, middle, furthest). We used the terms closest, middle, and furthest to designate the tapping regions in reference to the index finger for each hold position (Figure 11). Three factors: flexibility, hand, and number of tapping region per hold, were counter-balanced and the rest were randomized to avoid carryover effects.

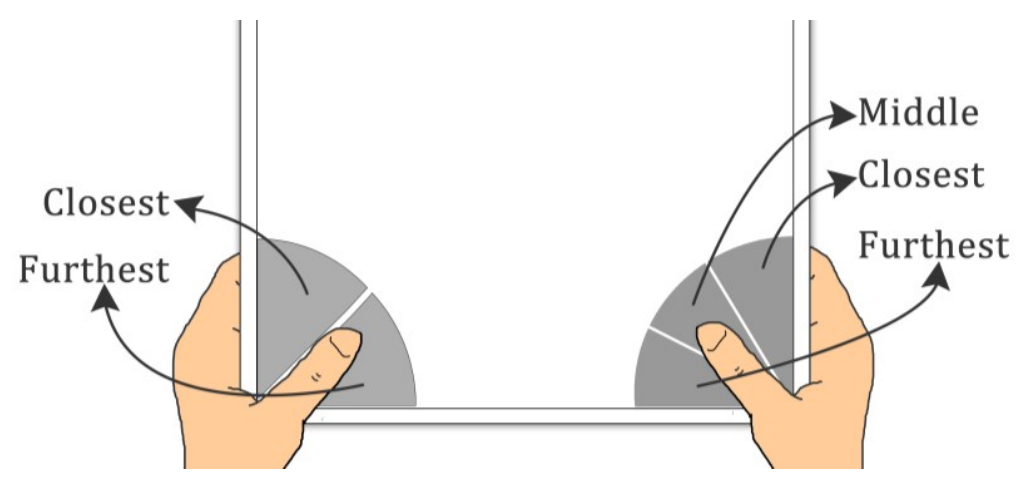

Figure 11: Designation of tapping regions 
To identify tapping regions, we used printed shapes (Figure 9), instead of dynamic projections to provide higher target location accuracy and to avoid visual occlusion. The current target was projected in the middle of the display (Figure 12), and the experimenter verbally told the participants about the type of shape (black or outlined), and which hand to use for interaction. The application visually informed the users about which hold position they needed to use for a particular task (Figure 12). 


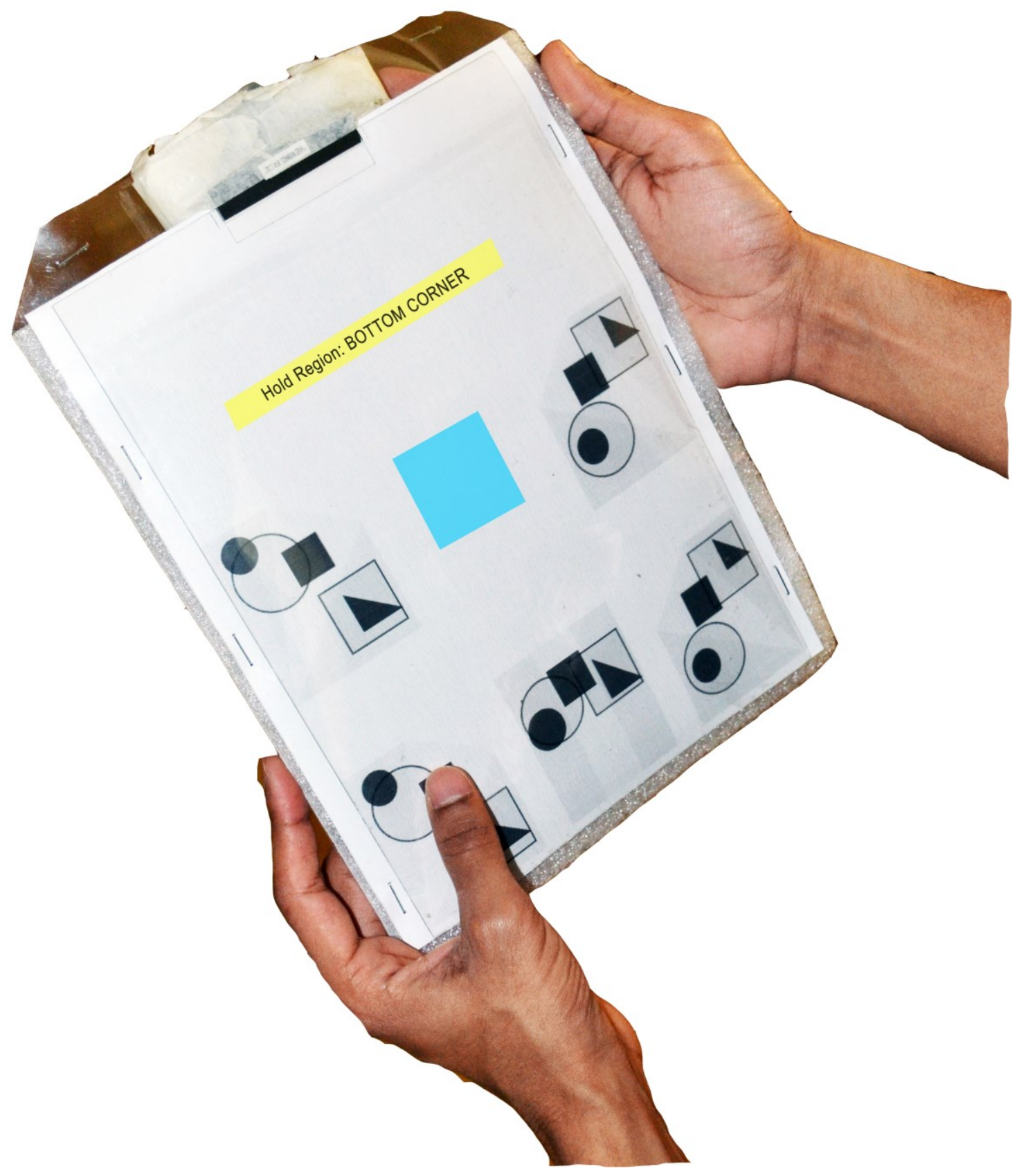

Figure 12: Tapping task using the flexible prototype 
All participants were instructed to hold the device in portrait mode (Figure 12) with minimum hand movement so that they could see the projection on the prototype surface without occlusion. They were instructed to use the holding hand for tapping. They were also required to use the other hand to provide additional support in holding the device on the top corner, as the size of the prototype required holding it with two hands. Participants were seated during the whole session (Figure 13)

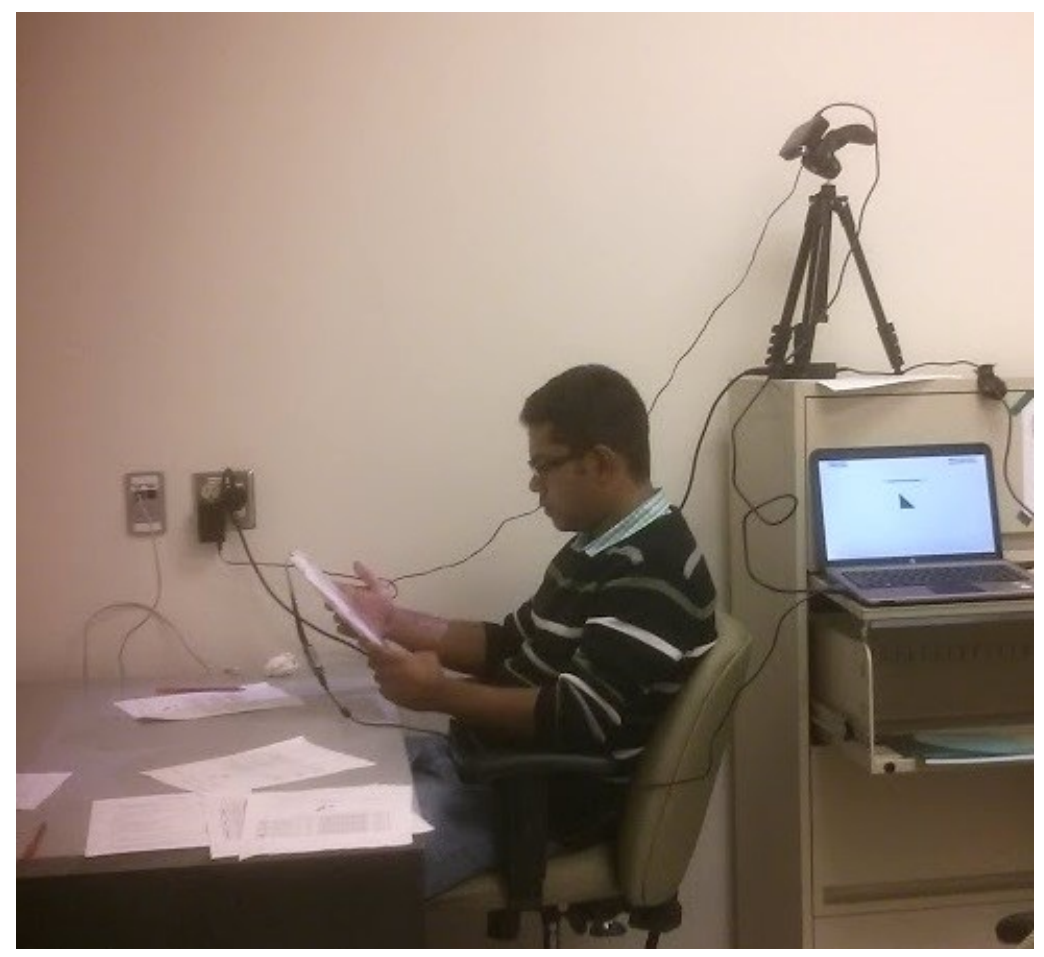

Figure 13: Participant is performing the tapping task

At the beginning of each session participants filled out demographic questionnaires. Later, they received a brief overview of the tasks, and were trained on how to hold the prototype and how to perform the tasks before starting the actual test. This pre-test session was about 10-15 minutes long.

The actual tapping test was divided into eight parts. In each part, we modified one of the three counterbalanced factors (number of tapping regions per hold (2), flexibility 
(2), hand (2)). Each part was comprised of three tasks: tapping in three hold positions (each task using one hold position) using either three regions or two regions, with one hand, with the flexible or the rigid prototype. Each of these eight parts of the tapping experiment was followed by a questionnaire about their interaction for that part of the test. The experimenter verbally informed the participants which hand they would use for tapping and the type of shape (black or outlined) they would tap.

Once the first part of the experiment started, the application visually informed the participants which hold position they needed to use. In the middle of the screen, the application showed the text "Start", which indicated to participants to activate the first task for that part of experiment (Figure 10). The participants were required to tap once anywhere in the touch area of the instructed hold position to activate the task. Once activated, the application displayed the shape that the participants were required to tap using the instructed hold position. After users tapped an appropriate shape, the application showed the next shape to tap within the same hold position. Each shape appeared five times for each hold position, in a random order. When users finished correctly tapping each shape for five times, the first task ended.

The application then indicated to change the hold position, and showed the text "Start" to activate the second task for that part of the experiment. The same procedure as in the first task was repeated for the new hold position, and then once again for the third hold position (third task). The order of hold position was randomized. After participants completed three tasks (i.e. tapping in three hold positions) for the first part of the experiment, the application showed the text, "Done!" at the middle of the screen. Then the experimenter handed out the questionnaires for this part of the tapping experiment to 
the participants. The questionnaires included questions about the difficulty level for each interaction (e.g. tapping the circle in the bottom center hold) using the 5 point Likert scale (1= Easy, 5 = Difficult). We also collected comments from participants to better understand their choices. Participants were allowed time to familiarize with the questions and the experimenter made sure that they understand how to answer the questions.

After participants filled out the questionnaires, the experimenter instructed them to hold the device again with both hands to start the second part of the tapping experiment following the exact same procedures as the first part, with a different, counterbalanced, set of conditions (flexibility, hand and number of tapping regions per hold). This was repeated for eight parts of the tapping experiment. The eight parts of the experiment together took about 20-25 minutes.

In total, participants performed 5 trials per combination of factors, for a total 300 trials (flexibility $(2) *$ hand $(2) *$ holds $(3) *$ total tapping regions $(5) *$ number of trial per interaction(5)). We measured the time to reach a target (the time between when the target is displayed and when the participant taps the relevant shape), and the number of errors (tapping a wrong target).

\subsection{Hypotheses}

We predicted that user preference and performance (duration and error) would be higher for two tapping regions per hold than three $(\mathrm{H} 1)$. We hypothesized that for three tapping regions per hold, the middle region would have the highest participant performance and preference across all holds for both hands and rigidities (H2). This hypothesis was based on the findings of Parhi et al. [25], where the researchers mentioned that the center target is the most preferred region for a $3 \times 3$ matrix. For two 
tapping regions per hold, we predicted that there would be no difference of user preference and performance between the two regions (H3).

We believe that flexibility would not have any significant effect on the performance or preference for any tapping interaction (H4). We also hypothesized that participants' performance and preference, for all tapping interactions, would be higher for the dominant hand than the non-dominant hand (H5), as generally participants are more efficient with their dominant hand. For tapping, we hypothesized that the participant preference and performance would be similar in bottom corner and side center hold, and bottom center will have significantly lower preference and performance (H6).

\subsection{Participants}

21 participants ( 8 females) completed our study. They were aged between 18 and 31 years $(M=24.5)$. Out of 21 participants, there were one left handed and one ambidextrous participant. All participants were familiar with touch screens and used them on a regular basis except one. Ten participants possessed a tablet and used it on a daily basis. Two of our participants had prior experience with a flexible display prototype. All the participants were university students and received $\$ 10$ gift card for an hour of their time.

\subsection{Results}

We performed a repeated measure ANOVA (A1), using the factors: flexibility (rigid, flexible), hand (dominant, non-dominant), hold (bottom corner, bottom center, side center). None of these three factors had significant impact on time to reach the target (Figure 14). Hand was the only a significant factor when looking at the number of errors 
$\left(\mathrm{F}_{1,20}=12.518, \mathrm{p}<0.01\right)$. Participants made more errors with the dominant hand than with their non-dominant hand. Table 1 provides the number of errors for the factors.

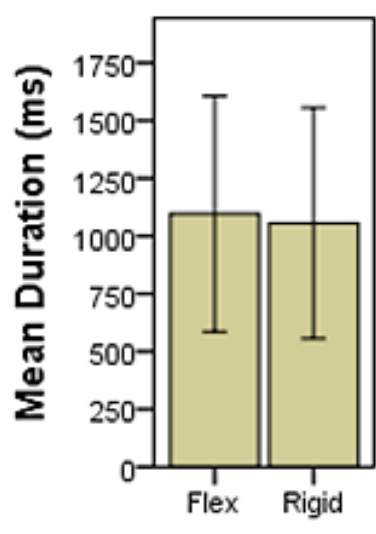

Flexibility

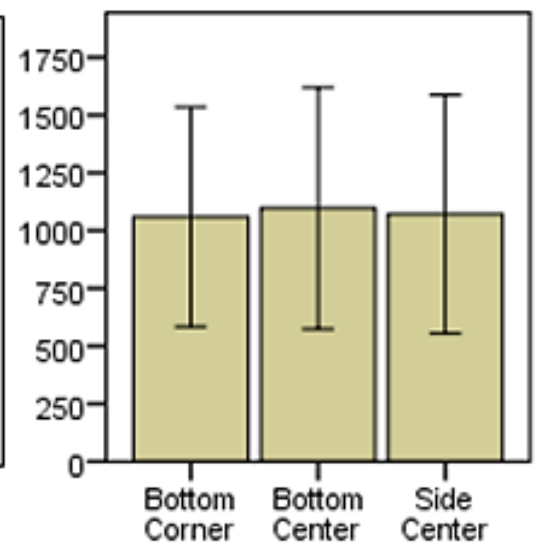

Hold Position

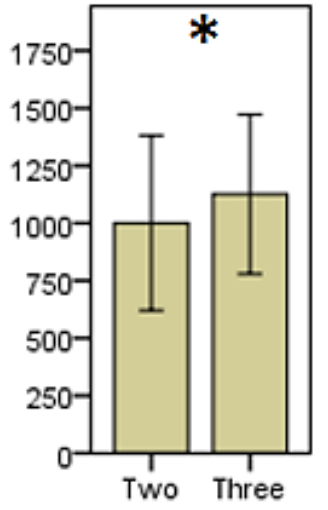

Reg./Hold

Figure 14: Mean tapping duration for flexibility, hold position and number of tapping regions per hold. Error bars represent standard deviation (SD). Asterisk indicates significance.

\begin{tabular}{|c|c|c|c|}
\hline \multirow{2}{*}{ ANOVAs } & \multirow{2}{*}{ Factor } & \multirow{2}{*}{ Level } & No. of Errors \\
\hline & & & Mean (SD) \\
\hline \multirow{7}{*}{ « } & \multirow{2}{*}{ Flexibility } & Flexible & $0.09(0.34)$ \\
\hline & & Rigid & $0.08(0.31)$ \\
\hline & \multirow{2}{*}{ Hand } & Dom. & $0.10(0.36)$ \\
\hline & & Non-Dom. & $0.07(0.28)$ \\
\hline & \multirow{3}{*}{ Hold } & Bottom Corner & $0.08(0.29)$ \\
\hline & & Side Center & $0.10(0.33)$ \\
\hline & & Bottom Center & $0.09(0.34)$ \\
\hline \multirow{2}{*}{ \& } & \multirow{2}{*}{ No. of tapping regions per hold } & Two & $0.10(0.25)$ \\
\hline & & Three & $0.08(0.17)$ \\
\hline \multirow{2}{*}{ 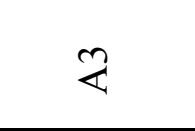 } & \multirow{2}{*}{ Two tapping regions per hold } & Closest & $0.06(0.32)$ \\
\hline & & Furthest & $0.13(0.39)$ \\
\hline \multirow{3}{*}{ 去 } & \multirow{3}{*}{ Three tapping regions per hold } & Closest & $0.08(0.30)$ \\
\hline & & Middle & $0.06(0.26)$ \\
\hline & & Furthest & $0.11(0.34)$ \\
\hline
\end{tabular}

Table 1: Performance data (number of errors) for tapping interaction. Bold indicates significance of the relevant factor. 
We performed a second ANOVA (A2) to analyze the effect of the number of tapping regions per hold (two, three) on tapping duration and error. For this ANOVA, we averaged the tapping region measures of two and three tapping regions per hold, for each trial, so we could compare the same number of measures. We found time was a significant factor $\left(\mathrm{F}_{1,20}=27.735, \mathrm{p}<0.001\right)$ : participants were faster with two tapping regions than three tapping regions per hold (Figure 14).

We performed a third ANOVA (A3) to investigate the impact of the active region of two tapping region per hold. We found significance on both time $\left(\mathrm{F}_{1,20}=13.621, \mathrm{p}<\right.$ $0.01)$ and error $\left(\mathrm{F}_{1,20}=8.475, \mathrm{p}<0.01\right)$. Participants were faster tapping the closest region $(\mathrm{M}=962 \mathrm{~ms}, \mathrm{SD}=454)$ than the furthest $(\mathrm{M}=1038 \mathrm{~ms}, \mathrm{SD}=521)$. The closest region also had lower number of errors (Table 1).

We ran a fourth ANOVA (A4) to detect the effect of active region of three tapping regions per hold. We found a significant impact on time $\left(\mathrm{F}_{2,40}=37.293, \mathrm{p}<\right.$ $0.001)$, and number of error $\left(\mathrm{F}_{2,40}=5.732, \mathrm{p}<0.01\right)$. The participants took the shortest time in the middle region $(M=1044 \mathrm{~ms}, \mathrm{SD}=416)$, then respectively the furthest $(\mathrm{M}=$ $1120 \mathrm{~ms}, \mathrm{SD}=506)$, and the closest region $(\mathrm{M}=1213 \mathrm{~ms}, \mathrm{SD}=578)$. A pairwise comparison using Bonferroni's correction confirms that all three regions are significantly different than each other for both duration $(p<0.01)$ and number of errors $(p<0.05)$. They made most errors in the furthest region, then in the closest region, and the middle region.

We performed a Friedman test using all the factors to analyze our Likert scale data about the comfort of tapping interaction. Participant preference significantly varied for the factors: flexibility $\left(\chi^{2}=10.121, p<0.01\right)$, hand $\left(\chi^{2}=10.195, p<0.01\right)$, hold $\left(\chi^{2}=\right.$ 
$170.629, \mathrm{p}<0.001)$, number of tapping regions per hold $\left(\chi^{2}=12.045, \mathrm{p}<0.01\right)$, and active regions of three tapping regions per hold $\left(\chi^{2}=6.743, \mathrm{p}<0.05\right)$.

Participants preferred the rigid version over the flexible one, and preferred two tapping regions per hold over three (Table 2). For three regions per hold, the middle region had the highest preference, then the closest and the furthest regions consecutively. The bottom center was the least preferred hold position while the side center and the bottom corner had close average ranks. The dominant hand had a higher preference than the non-dominant hand.

\begin{tabular}{|c|c|c|}
\hline \multirow[t]{2}{*}{ Factor } & \multirow[t]{2}{*}{ Level } & $\begin{array}{c}\text { Preference } \\
\text { Rank (Friedman) }\end{array}$ \\
\hline & & Mean (SD) \\
\hline \multirow{2}{*}{ Flexibility } & Flexible & $1.69(0.97)$ \\
\hline & Rigid & $1.62(1.01)$ \\
\hline \multirow{2}{*}{ Hand } & Dom. & $1.61(0.98)$ \\
\hline & Non-Dom. & $1.70(0.99)$ \\
\hline \multirow{3}{*}{ Hold } & Bottom Corner & $1.43(0.78)$ \\
\hline & Side Center & $1.40(0.72)$ \\
\hline & Bottom Center & $2.13(1.21)$ \\
\hline \multirow{2}{*}{ No. of tapping regions per hold } & Two & $1.56(0.89)$ \\
\hline & Three & $1.73(1.04)$ \\
\hline \multirow{2}{*}{ Two tapping regions per hold } & Closest & $1.52(0.90)$ \\
\hline & Furthest & $1.54(0.89)$ \\
\hline \multirow{3}{*}{ Three tapping regions per hold } & Closest & $1.78(1.05)$ \\
\hline & Middle & $1.64(0.98)$ \\
\hline & Furthest & $1.78(1.08)$ \\
\hline
\end{tabular}

Table 2: Subjective data for tapping interaction. Bold indicates significance of the relevant factor. 


\subsection{Tapping Heat Maps}

We created heat maps using the tapping points recorded throughout the sessions. Figure 15 displays them, grouped by two and three regions per hold. The darker regions of the heat maps represent higher point density.
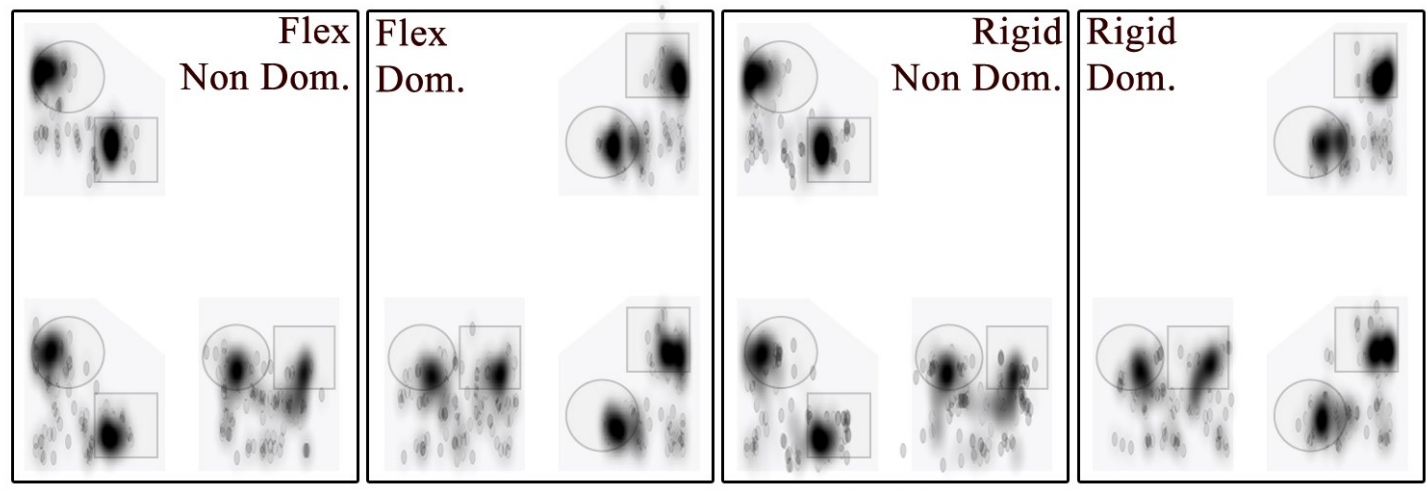

Two regions per hold
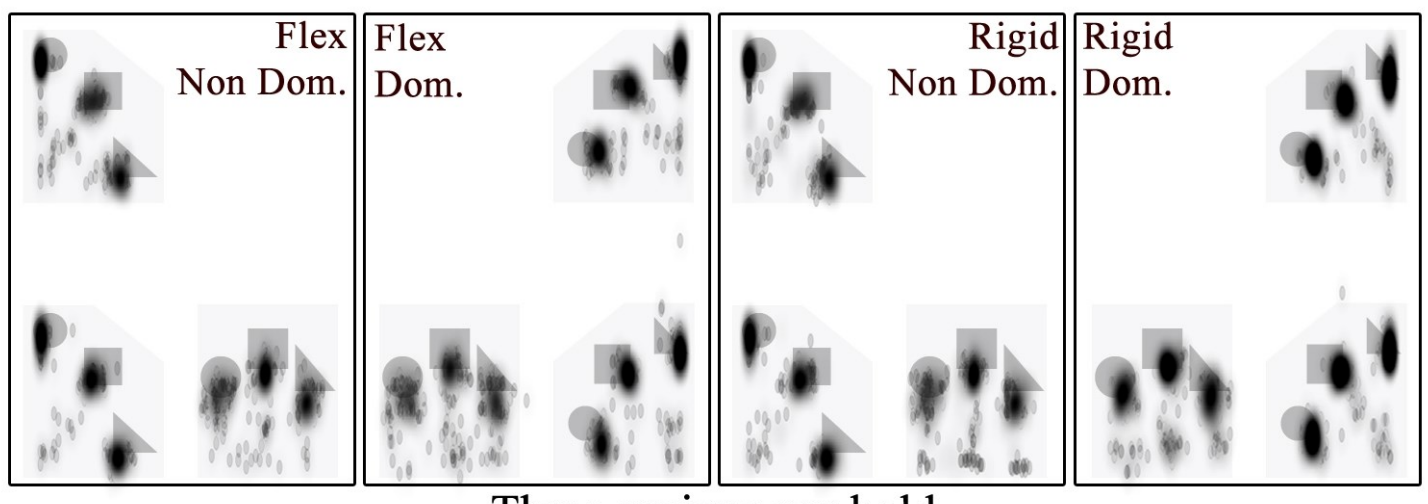

Three regions per hold

Figure 15: Heat maps for two and three tapping regions per hold for three holds, both rigidities, and both hands.

Heat maps for both two and three tapping regions per hold show that the participants mostly tapped the portion of the shapes that were closer to their hands, and rarely tapping the distant portion of the shapes. We also observed that for both two and three tapping regions per hold, darker regions did not overlap for adjacent tapping regions. For all conditions, heat maps show that the tapping points are more spread out in the bottom center hold than the other two holds. We noticed that the darker regions are 
bigger in the two tapping regions per hold than three, probably because of the wider acceptable region. We did not notice any major visual difference among similar tapping interactions for flexible and rigid version of the prototype and for the dominant and the non-dominant hand.

\subsection{Discussion}

\subsubsection{Flexibility}

Participants preferred the rigid version of the prototype over the flexible one for tapping interaction. Three participants specifically mentioned that the rigid prototype provided better support to hold the device. This result is expected, as it is easier to touch a rigid surface than a flexible one due to the constant normal force available [4]. This result partially violates our hypothesis $\mathrm{H}$, which states that flexibility would not have any significant effect on participant performance or preference for tapping interaction.

However, the performance analysis of flexibility for tapping interaction partially supports $\mathrm{H} 4$ as the two rigidities were similar in performance (duration and number of error). We also did not observe any visual distinction between the two rigidities in the heat maps. These results are encouraging, and indicate the potential of thumb input, to provide suitable touch interactions on flexible display. One participant, who preferred the rigid prototype for tapping, said, "The flexible one is too flexible". Another interesting comment by one participant identified that, "With the flexible one, it is easier to reach the distant shape, because the device gets bent with the hold and brings the distant shapes closer to hand". 


\subsubsection{Tapping Regions}

Participants were faster in two tapping regions per hold than three, and they preferred two tapping regions per hold over three, as hypothesized (H1). However, the error rates for tapping interaction did not vary significantly between two and three tapping regions per hold, which does not support H1. Overall, this result is not surprising, as two regions per hold allowed participants a wider area for tapping, and they also required making comparatively less thumb movement for two regions per hold than three. Two participants mentioned that they preferred two tapping regions per hold for nondominant hand but they were equally comfortable for two and three tapping regions per hold when they used their dominant hand.

One of our main concerns about two and three regions per hold was the error rates, which did not vary significantly for these two types of tapping task. This implies that participants had similar accuracy for distinguishing the adjacent tapping regions of both two and three regions per hold. From heat maps, we noticed that the participants rarely tapped the empty spaces between the shapes of both two and three tapping regions per hold, which also indicates that participants could successfully distinguish the adjacent tapping regions in both cases.

For three tapping regions per hold, both participant performance (duration and error) and preference were the highest at the middle region, which supports our second hypothesis. This finding is in line with prior research [25]. One participant said that the shapes that were closer to the hand were easy to reach than the ones away from the hand. The heat maps also explain this comment as we observed that participants mostly tapped the portion of the shapes closer to the hand and rarely touched the distant portions. 
As hypothesized (H3), for two tapping regions per hold, participants did not show any variation in preference between the two regions. However, participants made more errors and took more time when using the furthest region than the closest one, which contradicts our hypothesis H3. This finding is similar to the results of Park et al. [26], where the researchers showed that distant thumb targets and the targets that need more angular movement by thumb, generally produce lower user performance and preference. The heat maps show that the participants tapped the portion of the shapes that were closer to the hold, rather than the distant portions.

\subsubsection{Hand Dominance}

We obtained contradictory results when it comes to hand dominance. In terms of performance, contrary to our hypothesis H5, participants were less accurate with their dominant hand (more errors), yet they preferred using their dominant hand. We believe that this means they can use their both hands well. Our statistical analysis also suggests that the users took similar time for tapping interactions with both dominant and nondominant hands. As most participants will use their left hand to hold the device, this will allow a majority of participants to make use of their right, dominant hand for more precise tasks, without penalizing the left handed participants. The heat maps did not show any noticeable difference of the location or density of tapping points between two hands.

\subsubsection{Holds}

As opposed to our hypothesis H6, our performance analysis shows that participants had similar performance across all the holds. However, participants had similar preference for side center and bottom corner hold, but they felt less comfortable with the bottom center hold, which is in line with our hypothesis H6. It is coherent to find 
that the side center and bottom corner holds have similar preference, as the holds are actually quite identical and participants hold the device at a similar angle (see Figure 2). On the other hand, bottom center hold is quite different: it requires a perpendicular hold, which participants found uncomfortable, and the hardest one to use.

Overall, this study reveals that users can perform tapping with the thumb of the holding hand on both flexible and rigid tablets with comparable performance. They can use their both hands well for tapping interaction, and they like to hold the display on the side or on the bottom corner while tapping with the thumb. Our finding also suggests that users can distinguish two or three adjacent targets per hold with ease. 


\section{Chapter: Experiment 2: Swiping}

In the second study, we investigated participant performance and preference for swipe interactions. We assessed four types of swipe on flexible and rigid versions of the prototype. We evaluated swipe interaction in three common holds, with both the dominant and non-dominant hand, and compared the participant performance and preference for each swipe interaction.

We followed the same approach as in the tapping study. The same participants completed this study, and both studies were done in a single session.

\subsection{Task and Design}

In this experiment, the factors were: flexibility (rigid or flexible), hand (dominant or non-dominant hand), hold positions (bottom center, bottom corner and side center), and type of swipe (horizontal, vertical, diagonal and radial). Two factors, flexibility and hand, were counter-balanced and the rest two were randomized to avoid carryover effects.

The static grey area that represents the touch regions was also the area for swipe interaction for each hold (Figure 9). Since we used the same prototype as in the previous study, the black and outlined shapes were present, but not used. None of the participants indicated this as an issue. The type of swipe to perform was projected in the middle of the display (Figure 16), and the experimenter verbally told the participants about which hand to use for interaction. The application also informed the participants about the hold position to use for a particular swiping task (Figure 16). 


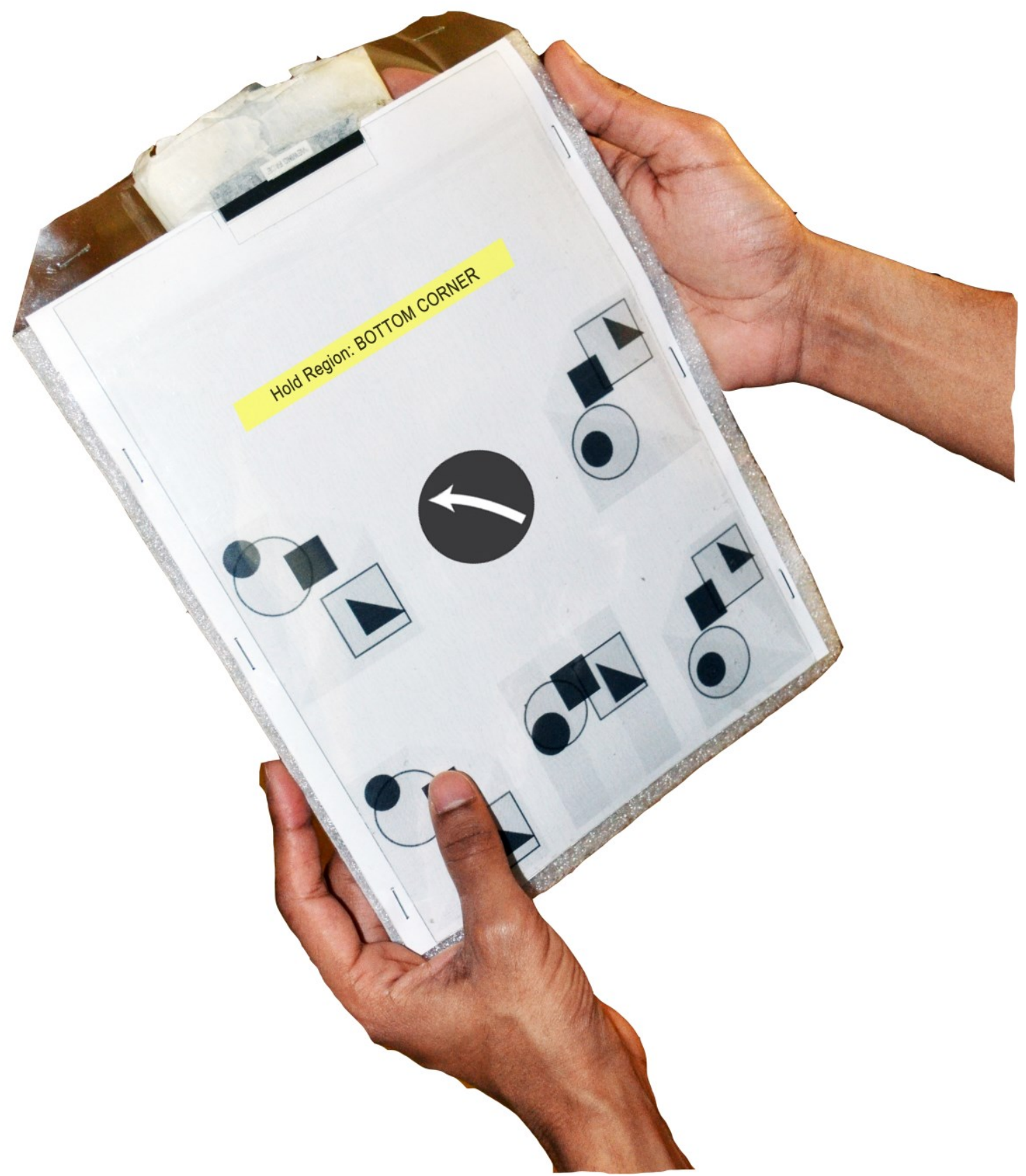

Figure 16: Swiping task using the flexible prototype 
We followed a similar procedure for swipe experiment as in tapping experiment. Before beginning the actual swiping experiment, participants were given a short training on how to use the prototype to perform swipe interaction. Participants were required to perform the swipes for a minimum length ( 9 pixel, or $2.38 \mathrm{~mm}$ in this case). Other than the required minimal length, participants were free to perform each swipe as they chose: there were no requirements for angle or direction, the only criteria being to perform a swipe within the appropriate region. This helped us to gain insight about the participant's preferred direction for each swipe. The experimental setup was exactly the same with tapping experiments. Participants were seated during the whole session.

The actual swiping test was divided into four parts. In each part of the swipe experiment, one of the two counter-balanced factors (flexibility(2), hand(2)) was different. Each part was followed by a questionnaire about their interaction for that part of the test.

Each part of swiping experiment was comprised of three tasks: performing swipes in three hold positions using one hand with either the flexible or the rigid prototype. The experimenter verbally informed the participants which hand they would use for swiping.

In the middle of the screen, the application showed the text "Start", which indicated to the participants to activate the first task of that part of swipe experiment. The participants were required to tap once on the adjacent touch area of the instructed hold position to activate the task. This activated the task and the application displayed a swipe at the middle of the screen that the participants were required perform using the instructed hold position. After users performed the swipe in the appropriate touch area, the application showed the next swipe to perform within the same hold position. Each 
type swipe (vertical, horizontal, diagonal and radial) appeared three times in each direction in a random order, for each hold position. When users finished correctly performing each swipe three times, the application indicated to change the hold position, and showed the text "Start". Participants would move the holding hand to the instructed hold position to start the second task for that part of the experiment and perform the swipes exactly in the same procedure they did for the previous hold position (first task).

This was repeated for three hold positions (three tasks). The order of hold position was randomized. After participants completed swiping in three hold positions, the application showed the text, "Done!" at the middle of the screen. This indicated the end of the first part of the swipe experiment. Then the experimenter handed out the questionnaires for this part of the experiment to the participants. The questionnaires included questions about the difficulty level for performing each swipe in each hold position (e.g. performing vertical swipe in side center hold) using the 5 point Likert scale $(1=$ Easy, 5 = Difficult $)$. We also collected comments from participants to better understand their choices.

After participants filled out the questionnaires, the experimenter instructed them to hold the device again with both hands to start the second part of the swipe experiment following the exact same procedures as the first part. This was repeated for four parts of the swiping experiment. These four parts of swiping experiment together took about 1520 minutes.

In total, participant performed 3 trials per combination of factors, for a total of 288 trials (flexibility $(2) *$ hand $(2) *$ holds $(3) *$ type of swipe $(4) * \operatorname{direction}(2) *$ number of trial(3)). We measured the duration of each swipe (time between when participants 
touch the appropriate region to start swipe and when they lift off their thumb) for our performance analysis.

\subsection{Hypotheses}

We predicted no significant difference in preference or performance between rigid and flexible versions of the prototype for swipe interaction $(\mathrm{H} 7)$. We hypothesized that the diagonal and the radial swipe would have lower participant performance (longer duration) and preference than the horizontal and the vertical swipe (H8), to be in line with the prior work [11]. We expected that the dominant hand would have significantly higher participant preference and performance (H9). We also predicted that participant preference and performance would be higher in bottom corner and side center hold than bottom center hold (H10).

\subsection{Result}

We performed a repeated measures ANOVA, using the factors: flexibility (2), hand (2), hold position (3), and type of swipe (4) on the duration data. We found statistical significance on swipe duration for hold $\left(\mathrm{F}_{2,40}=27.276, \mathrm{p}<0.001\right)$, and hand $\left(\mathrm{F}_{1,20}=5.163, \mathrm{p}<0.05\right)$

Figure 17 illustrates the mean swipe duration for these factors. Participants took significantly longer time to swipe in the bottom center hold position ( $\mathrm{M}=220.04 \mathrm{~ms})$ than the side center $(\mathrm{M}=161.69 \mathrm{~ms})$ and the bottom corner hold $(\mathrm{M}=159.59 \mathrm{~ms}) . \mathrm{A}$ pairwise comparison using Bonferroni correction $(\mathrm{p}<0.001)$ confirmed this variation of swipe duration among the bottom center hold and the other two holds. We found that the participants were significantly faster with their dominant hand $(\mathrm{M}=170.38 \mathrm{~ms})$ than with their non-dominant hand $(\mathrm{M}=190.49 \mathrm{~ms})$. 


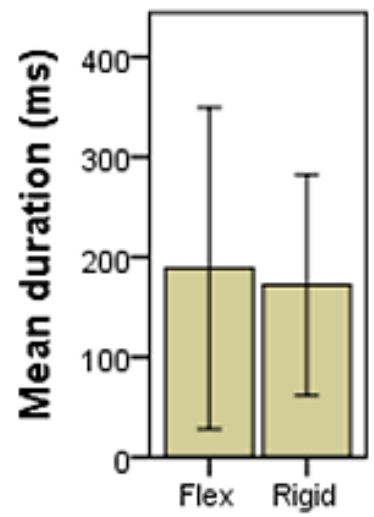

Rigidity

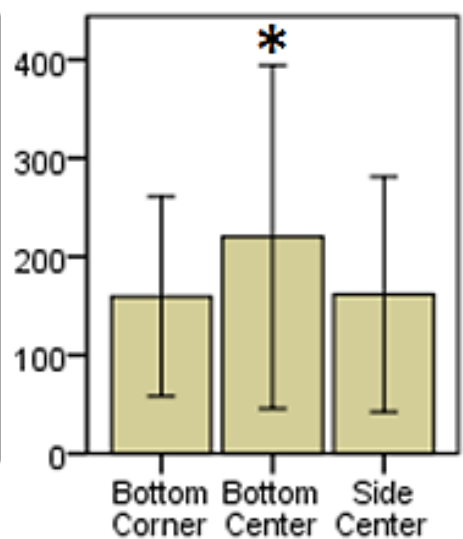

Hold Position

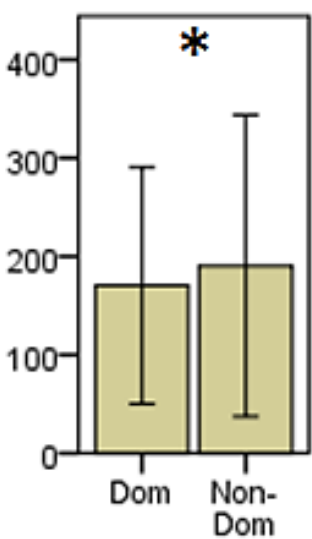

Hand

Figure 17: Mean swipe duration for rigidity, hold position, and hand. Error bars represent standard deviation. Asterisks indicate significance.

There was no statistical difference in swipe duration between the flexible and the rigid prototype $\left(F_{1,20}=3.512, p>0.05\right)$. The average duration for each type of swipe did not vary significantly $\left(\mathrm{F}_{3,60}=2.648, \mathrm{p}>0.05\right)$. The average durations for diagonal $(\mathrm{M}=$ $190.95 \mathrm{~ms}, \mathrm{SD}=148.54)$ and radial swipe $(\mathrm{M}=186.28 \mathrm{~ms}, \mathrm{SD}=139.40)$ were slightly higher than the horizontal $(M=173.64 \mathrm{~ms}, \mathrm{SD}=133.51)$ and vertical swipe $(\mathrm{M}=170.85$ $\mathrm{ms}, \mathrm{SD}=129.04)$.

We analyzed our Likert scale data for the same factors using the Friedman test. The significant factors were: type of swipe $\left(\chi^{2}=74.996, p<0.001\right)$, hold position $\left(\chi^{2}=\right.$ 93.071, $\mathrm{p}<0.001)$, and hand $\left(\chi^{2}=39.7, \mathrm{p}<0.001\right)$. Flexibility did not have any significant $\left(\chi^{2}=0.068, p>0.05\right)$ impact on participant preference.

Table 2 shows that participants preferred the horizontal swipe, followed by the vertical, radial and diagonal swipe, consecutively. Participants preferred swiping using the side center and bottom corner hold position over the bottom center hold position. Participant preference was higher for the dominant hand than the non-dominant hand. 


\begin{tabular}{|c|c|c|c|}
\hline \multirow{2}{*}{ Factor } & \multirow{2}{*}{ Levels } & \multicolumn{2}{|c|}{ Preference Rank } \\
\cline { 2 - 4 } & & Mean & SD \\
\hline \multirow{2}{*}{ Flexibility } & Flexible & 1.90 & 1.22 \\
\cline { 2 - 4 } & Rigid & 1.85 & 1.14 \\
\hline \multirow{2}{*}{ Hand } & Dominant & $\mathbf{1 . 7 1}$ & $\mathbf{1 . 0 5}$ \\
\cline { 2 - 4 } & Non-Dominant & $\mathbf{2 . 0 4}$ & $\mathbf{1 . 2 8}$ \\
\hline \multirow{2}{*}{ Hold } & Bottom corner & $\mathbf{1 . 6 6}$ & $\mathbf{1 . 0 3}$ \\
\cline { 2 - 4 } & Side center & $\mathbf{1 . 6 9}$ & $\mathbf{1 . 0 7}$ \\
\cline { 2 - 4 } & Bottom center & $\mathbf{2 . 2 7}$ & $\mathbf{1 . 3 3}$ \\
\hline \multirow{3}{*}{ Type of swipe } & Diagonal & $\mathbf{2 . 3 0}$ & $\mathbf{1 . 4 0}$ \\
\cline { 2 - 4 } & Horizontal & $\mathbf{1 . 6 0}$ & $\mathbf{0 . 9 2}$ \\
\cline { 2 - 4 } & Radial & $\mathbf{1 . 8 3}$ & $\mathbf{1 . 1 6}$ \\
\cline { 2 - 4 } & Vertical & $\mathbf{1 . 7 6}$ & $\mathbf{1 . 0 9}$ \\
\hline
\end{tabular}

Table 3. Subjective data for swipe interaction. Bold indicates significance.

\subsection{Swipe Point Cloud Maps}

Using the swipe touch points collected during each session, we created point clouds and regression lines for four types of swipe for different flexibility, hand, and hold position. Figure 18 illustrates those point cloud maps. Appendix B contains the values for regression line equations.

Excluding the vertical swipe type, the regression lines show that swipes performed in the bottom center hold tend to be less steep than the other two holds, i.e. the regression line is less angled in reference to the $\mathrm{x}$-axis. There is a variation in steepness for the diagonal swipe between each hand: the regression lines are steeper for the dominant hand than the non-dominant hand. 


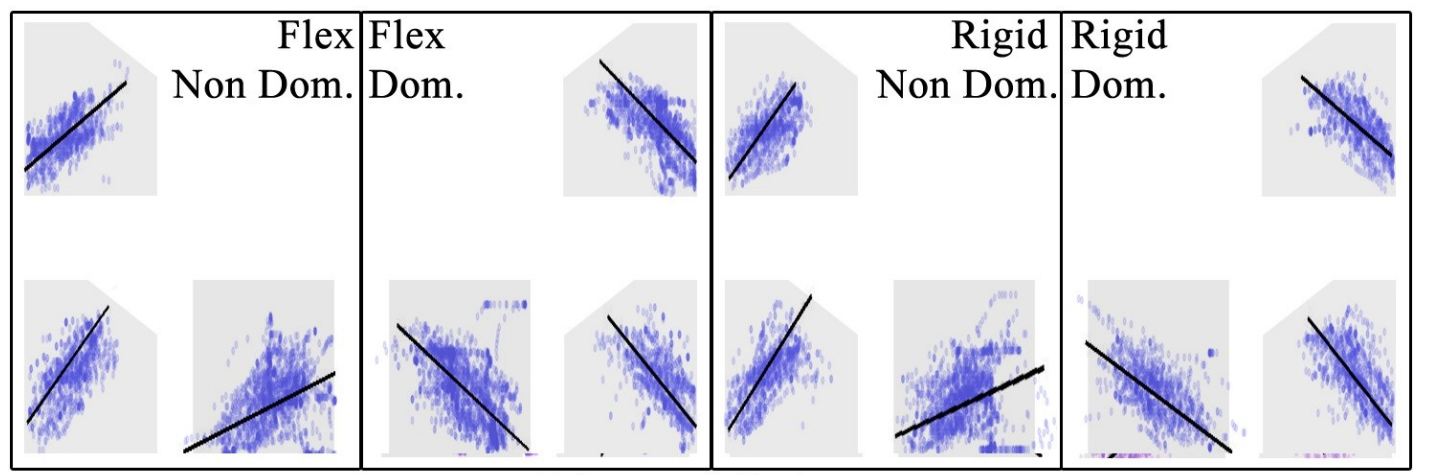

Diagonal Swipe

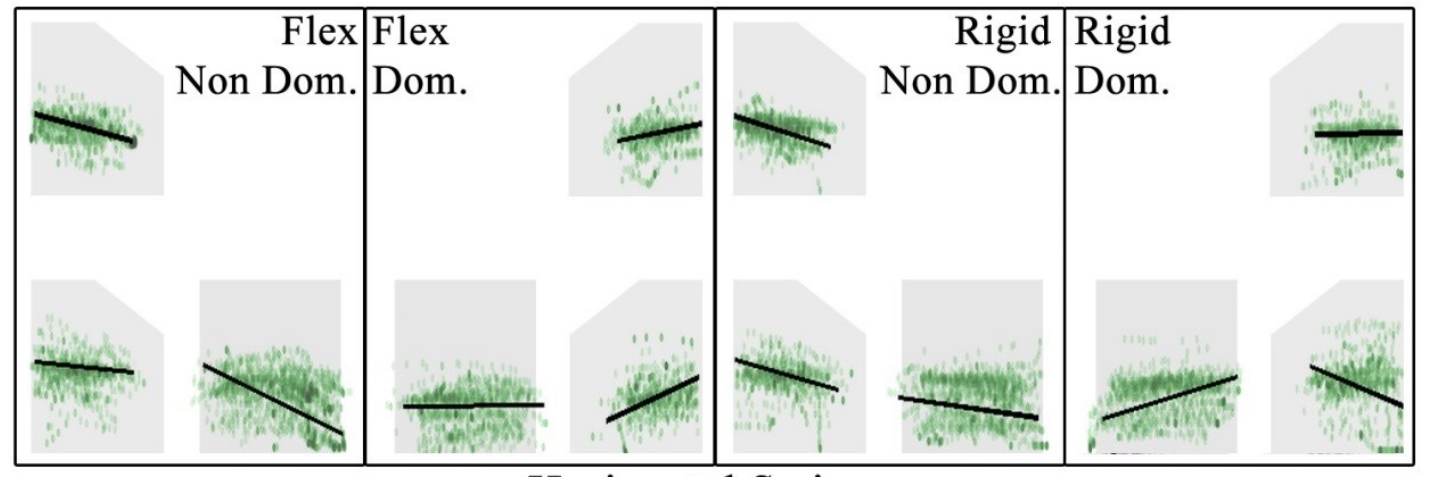

Horizontal Swipe

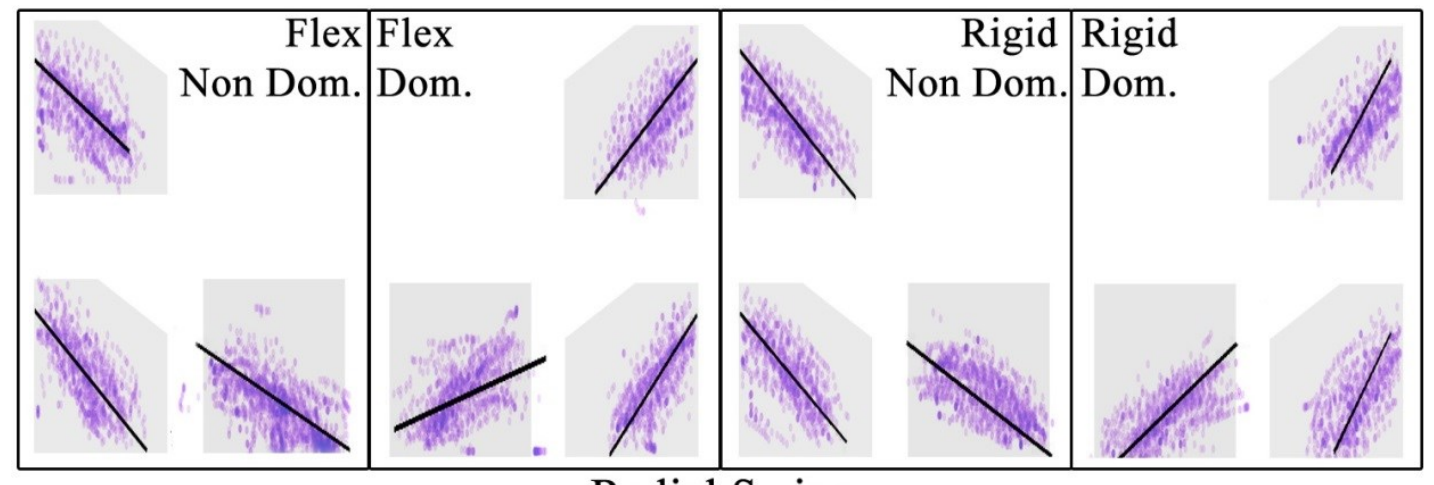

Radial Swipe

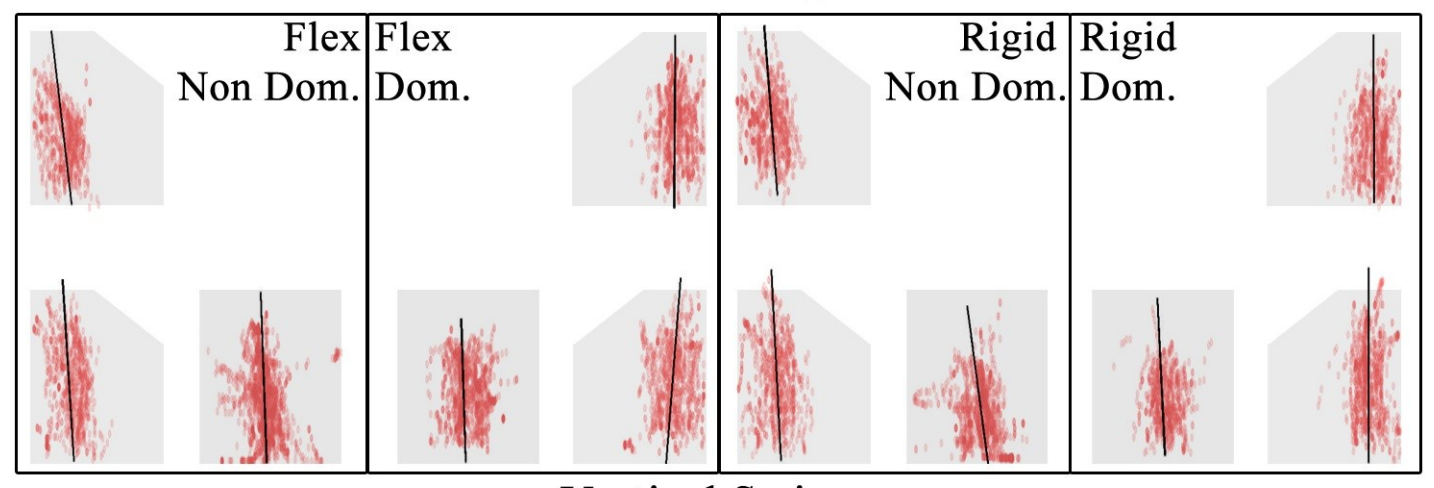

Vertical Swipe

Figure 18: Point cloud maps for four types of swipe for all holds, both rigidities and both hands. The regression line of each hold is indicated with a black line. 
We also notice that for all four types of swipe, swipe points are closer to the edge of the prototype for the bottom center hold than in other two holds. We explain this as the users have a more comfortable range of thumb movement in the vertical direction for the side center and bottom corner holds than the bottom center hold, which is in line with prior work [3]. It is also visible that the swipe touch points in the bottom center hold are more spread out in horizontal direction than the other two holds.

For horizontal swipes, regression lines show that the participants failed to do a straight swipe in most cases. Especially in the bottom center hold, the horizontal swipes are angled in a similar direction as the radial swipes. The vertical swipes are, however, very close to being perfectly vertical.

We did not notice any major visual difference in swipe point clouds for flexibility. We believe these point cloud maps to be particularly useful for design decisions about which swipes to integrate, and for developing the detection algorithm to identify them.

\subsection{Discussion}

\subsubsection{Flexibility}

Our analysis shows that participants had similar performance and preference for swipe interaction for both flexible and rigid prototypes, which confirms our hypothesis $\mathrm{H}$, where we stated that flexibility will not have significant effect on participant preference and performance for swipe interaction. We also did not notice any visual distinction of the swipes for different flexibility in the point cloud maps.

Five participants who preferred the rigid prototype for swipe, stated that it provided them with better control to hold the device. Five other participants, who preferred the flexible prototype, also mentioned similar reasons to opt for the flexible 
version. Two participants, who were more comfortable with the flexible prototype, provided interesting comments about flexibility, stating that the device fits into the hand a bit better, increasing the range of thumb. They mentioned the same observation in the tapping task.

\subsubsection{Type of Swipe}

Swipe duration did not vary significantly among four types of swipe in general, although we did observe that the diagonal and radial swipes were systematically taking longer time than their vertical and horizontal counterparts. Participant preference significantly varied across different swipes. The diagonal swipe was much less appreciated by the participants than the other three types of swipe, which supports our hypothesis H8. This finding is in line with the previous research about thumb interactions in a rigid handheld device [11]. One participant commented, "Diagonal swipe was painful for fingers", also supporting Karleson et al.'s results [11].

The point cloud maps for swipes show that all four types of swipe are visually identifiable, which indicates that the participants could distinguish the swipes and perform them accordingly. We could expect these swipes to be easily distinguishable using an automatic classification algorithm. However, we did notice that horizontal swipes were not as horizontal, or even straight, as expected. In some cases, particularly in the bottom center hold, some of the horizontal swipes overlapped with the radial ones. This may lead to a classification issue. Interestingly, we did not receive any participant feedback supporting this interference; only one participant thought that diagonal and vertical swipes were close. 


\subsubsection{Hand Dominance}

As hypothesized (H9), participant performance and preference for swipe were better with their dominant hand than their non-dominant hand. This result was expected. However, we do not think this means users cannot use their non-dominant hand for swipe tasks at all. We believe this indicates that users are less efficient and less comfortable with their non-dominant hand than their dominant hand, but they can still utilize both hands for swipe interaction when required. For instance, users may want to switch hands while using a flexible tablet to avoid fatigue on one hand.

The point cloud maps do not show any noticeable visual variation of swipes across the two hands, except for the diagonal swipes: they are steeper in bottom center hold with the dominant hand than with the non-dominant hand.

\subsubsection{Holds}

Participants took the longest time to swipe in the bottom center hold position. Their preference for hold position for swipe interaction was also similar to their performance, which implies that our hypothesis H10 is true for both objective (performance) and subjective (ease of use) data. This is coherent with the tapping experiment. The point cloud maps indicate how all the swipes are visually different in the bottom center hold than the other two holds. They also indicate how participants overlapped horizontal and radial swipe in bottom center hold. The bottom center hold allows more thumb movement in the horizontal direction, whereas the other two holds provide more room for vertical thumb movement [3]. This is because of the pattern of the swipe is different for the bottom center hold than the other two holds. For swipe 
interaction, this evidence establishes the bottom center hold as a different hold than the other two. 


\section{Chapter: Discussion}

In this chapter, we provide design recommendations to integrate thumb input on flexible displays. These design recommendations are based on our findings from the experiments on tapping and swiping. Based on the design recommendations, we provide three use case scenarios to show some potential usage of thumb input on flexible tablets.

\subsection{Design Recommendations}

The findings from the tapping experiment and swiping experiment can be summarized in the following design recommendations to incorporate thumb input on flexible tablets:

\subsubsection{Flexibility}

Our participants had comparable performance for both rigid and flexible prototypes, for both tapping and swiping interactions. Participants also showed similar preference for swipe interaction in both prototypes. Though the user preference for tapping interaction was higher in the rigid prototype, we believe this lower preference will be counteracted by bringing the benefits of touch input on flexible displays. We also believe this preference to be further improved when touch input will be used in parallel with bend gestures, as combining these two input modalities improves user experience in deformable displays [14].

Our results from both experiments are encouraging and leverage the compatibility of touch input on flexible displays with the thumb of the holding hand. Hence, we recommend using thumb input on flexible handheld devices to bring the advantages of touch input in flexible displays. 


\subsubsection{Hand Dominance}

For tapping interaction, although the participants preferred using the dominant hand, they also made more errors with that hand. Our statistical analysis also suggests that users took a similar amount of time for tapping with either hand. We believe that these contradictory results imply that the users can use either hand well for tapping interaction. We recommend utilizing both hands for tapping interaction, as it gives users the opportunity to use either or both hands according to their comfort and context of usage.

Participant's preference and performance for swipe interaction was higher with the dominant hand, which may lead some to recommend doing swiping with the dominant hand only. However, holding the device using the non-dominant hand will allow users to perform other operations with the dominant hand, whether additional touch interactions, or bend interactions, which are preferred on the top right corner [16,33]. Hence, we believe that even given those results, the benefits of offering swipe interaction with both hands outweighs the downsides, as it allows users to switch hands during using a flexible tablet to avoid fatigue on the dominant hand.

\subsubsection{Hold Position}

For both tapping and swiping, the bottom center was the least favored hold position by the participants. In addition, this region also resulted in slower swipes. This finding is consistent with the previous research with a tablet sized rigid device where the authors found that the thumb interaction was more difficult in the bottom center hold than the bottom corner and the side center hold [32]. We discourage the use of this hold position for both interaction techniques. 


\subsubsection{Tapping Regions}

Though participants preferred two tapping regions per hold over three, they distinguished the adjacent tapping regions for both two and three regions per hold with comparable accuracy. Given that three tapping regions offer more functionality to the user, we suggest that both two and three tapping regions can be integrated for thumb touch input.

For three tapping regions per hold, we recommend assigning the most frequently used tapping zone to the middle region, as participants had the highest performance and preference in this region.

Our estimated calculation suggests (see Figure 3) that users can reach up to an average distance of approximately $4.5 \mathrm{~cm}$ from the outer edge of the screen using the thumb of the holding hand for three common hold positions (see Figure 3). We recommend to avoid placing tapping targets beyond that distance from the edge of the display for these three common holds.

\subsubsection{Type of Swipe}

We recommend avoiding the diagonal swipe for all three holds, as it was the least preferred swipe across all hold positions. We suggest that the horizontal, radial and vertical swipes can be implemented for side center and bottom corner hold. According to our point cloud maps, the horizontal and the radial swipe may overlap with each other on the bottom center hold. As mentioned in 7.1.3, we do not recommend the use the bottom center hold. If the application requires this hold, we recommend using only horizontal and vertical swipes for this hold. 


\subsection{Use Case Scenario}

In this section, we discuss three use case scenarios to show the potential usage of touch input using the thumb of the holding hand on flexible displays. We chose to illustrate three common tasks for handheld tablets: e-book reading, icon navigation, and photo editing. The first two are simple applications with limited functionalities, using two tapping regions per hold, while the photo editing scenario involves a more complex application, with concurrent and combined functionality that may use the same interaction in different contexts, with three tapping regions.

The use case scenarios are based on our design guidelines on thumb input for flexible displays. We made some additional design assumptions for the representation of the use case scenarios; therefore, they must be investigated before implementation:

- The touch regions will be shown as transparent overlay.

- Double tapping anywhere on the screen will activate or deactivate the touch regions. Upon activation, the transparent touch regions will be visible on the screen.

- Ten seconds of inactivity of interaction will auto-hide and auto-deactivate the transparent touch regions.

\subsubsection{E-Book Reading}

Reading e-books is a common usage of handheld tablets. Figure 19 illustrates the user interface of an e-book reading application with the transparent touch regions for thumb input on flexible tablets.

Utilizing thumb input on flexible tablets, users can use radial swipes to move to the next or the previous page of the book. Following a book metaphor [34], a radial swipe 
in the left direction will take users to the next page, and the right direction to the previous page. If users want to scroll faster instead of navigating page by page, they can use a horizontal swipe. For instance, a horizontal scroll in the left direction can take users to a page that is ahead by five pages than the current page. We also envision using the speed of swipe to have effect on fast scrolling. For example, a faster horizontal swipe can help users navigate by ten pages.

The tapping regions are marked with contextual icons according to their operation. Tapping the 'home' icon takes users to the home screen of the tablet operating system, whereas, the other icon, labeled with 'collection', takes them to their e-book library.

In this simple application, each hold for each hand (i.e. the four touch regions) offers the same functionality (next/previous page for swiping, home/library for touching). We made this design choice to leverage the comfort of interaction [32]. This helps the users avoid changing the hold position too often for accessing different functionalities. However, they can use any hold they want and still able to do everything from one hold. 


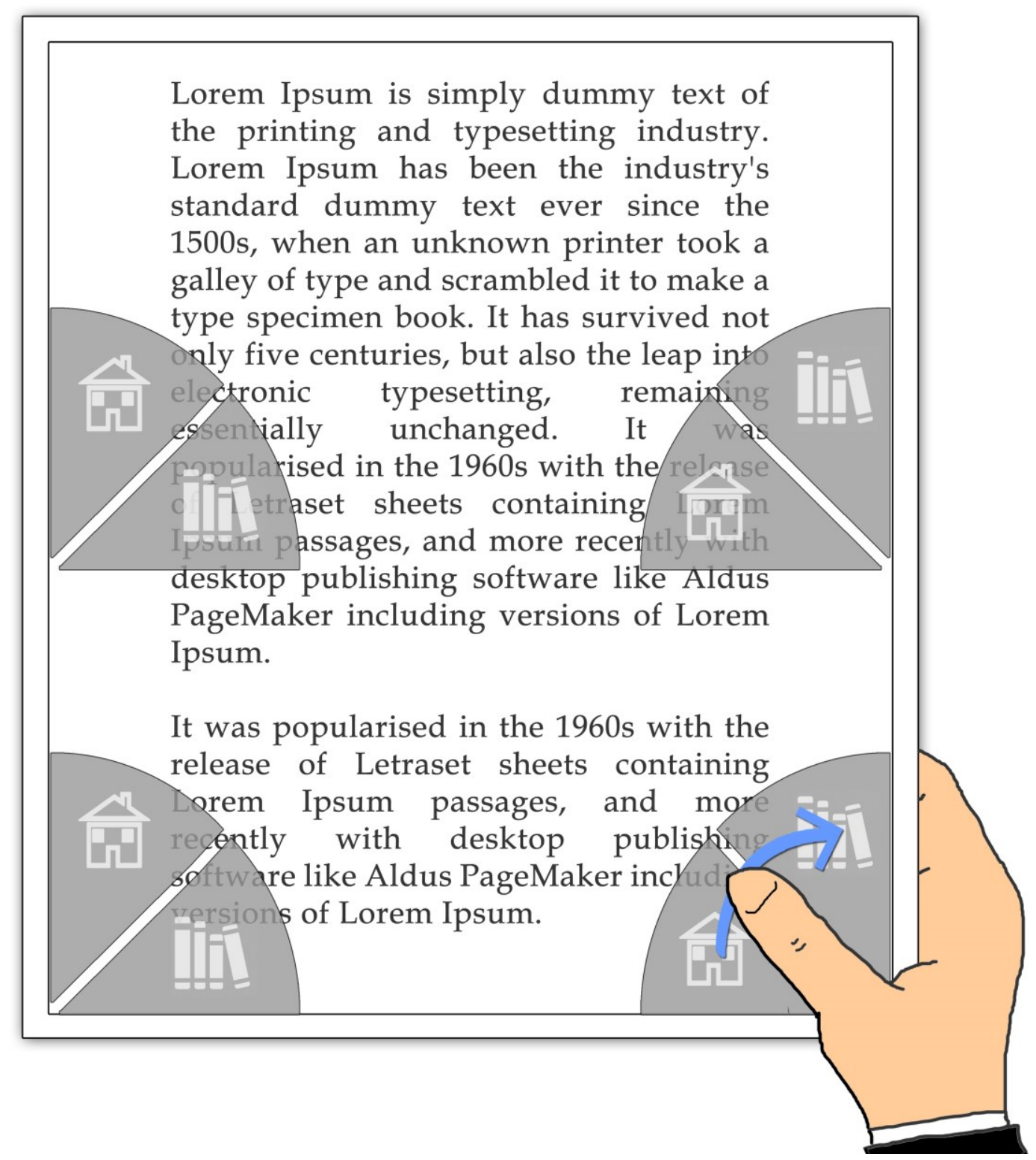

Figure 19: In an e-book reader, the user can use radial swipe with thumb input to navigate to the next or previous page (blue arrow). The user can also touch the tapping region of one of the two icons to either go to the home screen, or to access their library (white icon in gray zone). 
For the e-book reading scenario, instead of showing all four available touch regions, another design choice could be displaying only one touch region. Figure 20 illustrates this design choice within the same e-book reader application.

According to our basic design choices for all scenarios, a double tap would toggle the activation of thumb input as well as the visualization of the contextual touch regions. In this e-book reader application, the users can access all the available options with one touch region. All four touch regions offer exactly the same functionality. Instead of showing four touch regions with the same options, we can visualize only one touch region in this context (Figure 20). We can use the same activation method for this design choice as well: using a 'double tap'. The closest touch region to the location of 'double tap' would be activated. While using the activated touch region, if the user decides to change the hold position, they can simply double tap in the adjacent area of the desired hold position to activate thumb input for that hold, which will also cause the previously activated touch region to be deactivated. Like the previous design choice, this allows users to change the hold position whenever they want, but with an extra 'double tap'. However, this design choice avoids visualizing the currently unused touch regions, which provides a less cluttered interface. 


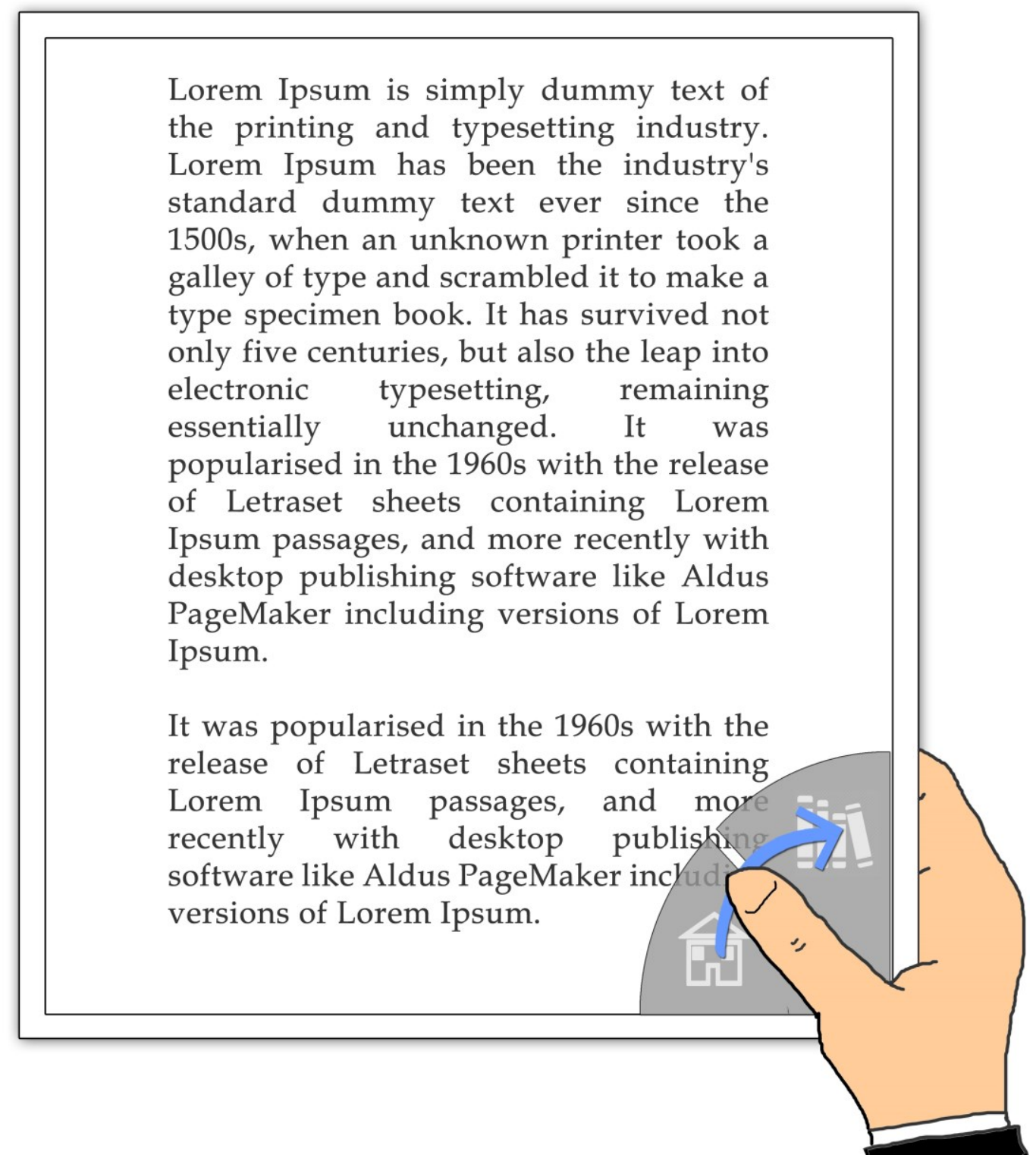

Figure 20: The user can double tap in the adjacent area of any hold region to activate thumb input and visualize touch regions only for that particular hold. They can use radial swipe with thumb input in that region to navigate to the next or previous page (blue arrow). 


\subsubsection{Icon Navigation}

Icon navigation is another simple and common task in many handheld tablets. Similar to the e-book reader scenario, each hold for each hand (i.e., total four touch regions) offers the same functionality. As a result, we can have the two design choices we had for e-booking reading scenario: showing all the four touch regions constantly, or showing one at a time. Figure 21 illustrates the user interface for icon naviagtion using thumb input on flexible tablets. It shows the user interface with a single touch region activated at a time.

For navigating the icons, users can use horizontal and vertical swipes. For instance, a horizontal swipe in the right direction will move the selection to the icon on the right. We can also utilize the speed of swipe to bring pace in navigation. For example, swiping more quickly in the same direction will move the selection by two icons to the right.

The tapping region marked with a 'folder' is used to open the selected application. The other icon labeled with 'menu' opens the contextual menu for the selected application. Contextual menus are not common in present day tablet operating systems but it is widely used in desktop computers. Instead, in tablet devices, users can typically access options within the application. With our design, we propose to save the step of opening the application to access the options. By placing the selection icon on the left tapping region, and the contextual menu on the right tapping region, we reproduce the button placement and functionality of mouse input in desktops. 


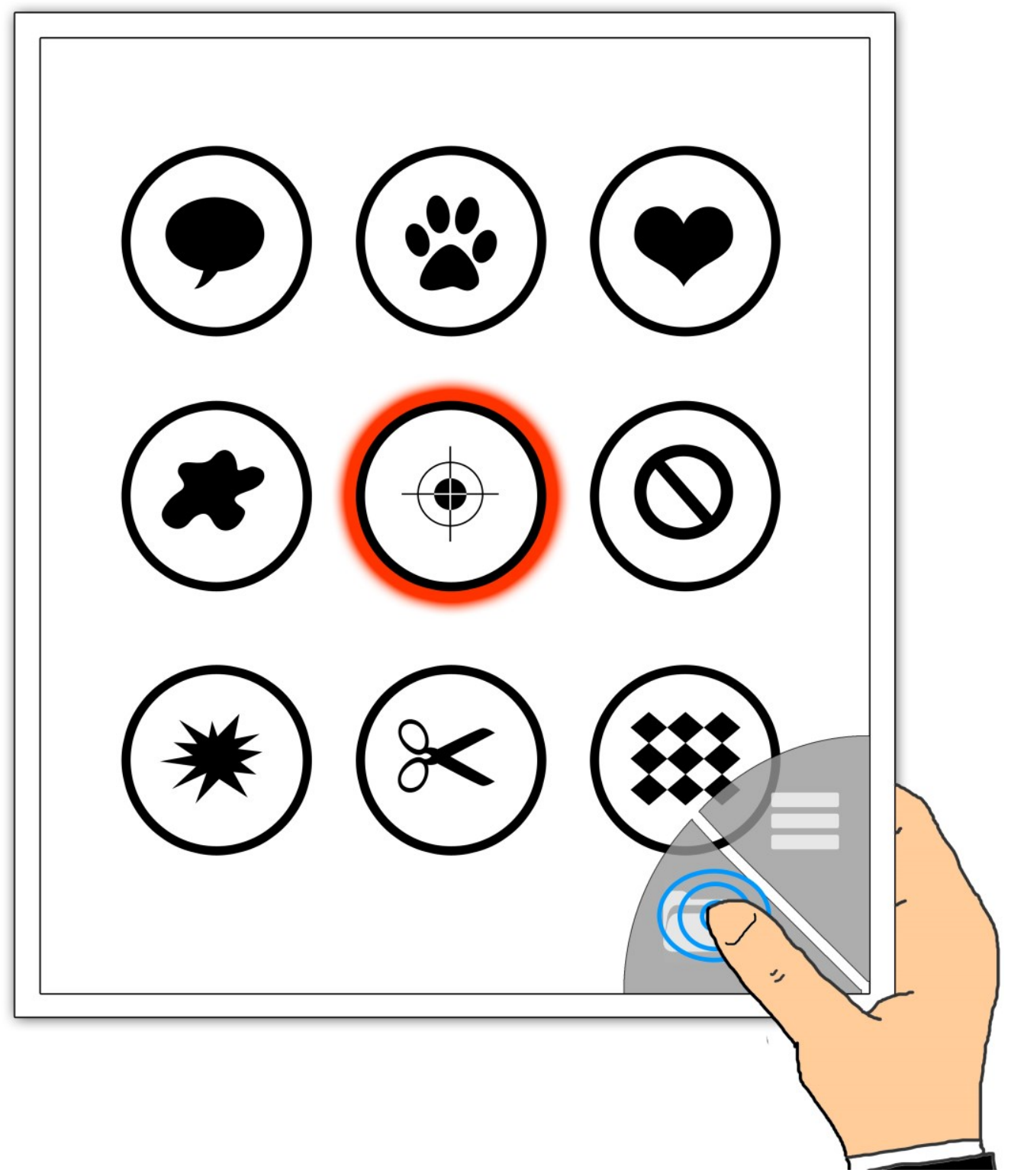

Figure 21: In a home screen displaying icons of applications, the user can tap the 'folder' icon using their thumb to open the selected application (highlighted in red). 


\subsubsection{Simple Photo Editing}

Simple photo editing applications for smartphones and tablets are becoming increasingly popular. In this scenario, in addition to single-handed usage of flexible tablets using the thumb of the holding hand, we illustrate how thumb input can be utilized for bimanual interaction. Figure 22 shows the user interface of a photo editing tool being used with single handed thumb input, and Figure 23 illustrates the bimanual usage using the same user interface. It displays the currently edited image in the middle of the screen, photo editing options (e.g. contrast, brightness, cropping) on the top, and transparent touch regions in four hold regions.

Unlike the e-book reading and icon navigation scenarios, this scenario offers different functionality for each hold per hand. This scenario also uses three tapping regions per hold.

\subsubsection{Single-handed Thumb Input Photo Editing Application}

The tapping regions of the bottom corner hold for both hands are used to navigate through photo editing options. As opposed to the previous two scenarios and our experimental design, in this hold, we mapped the tapping regions symmetrically for both hands. This means the tapping regions for the left hand for bottom corner hold is the mirror image of the tapping regions for the right hand for that hold. We made this design choice for this specific context, where three tapping regions in bottom corner hold are assigned for navigating the photo editing options: using the tapping regions labeled with next/previous icons to select the next/previous photo editing options, and using the middle tapping region to activate the current selection. 


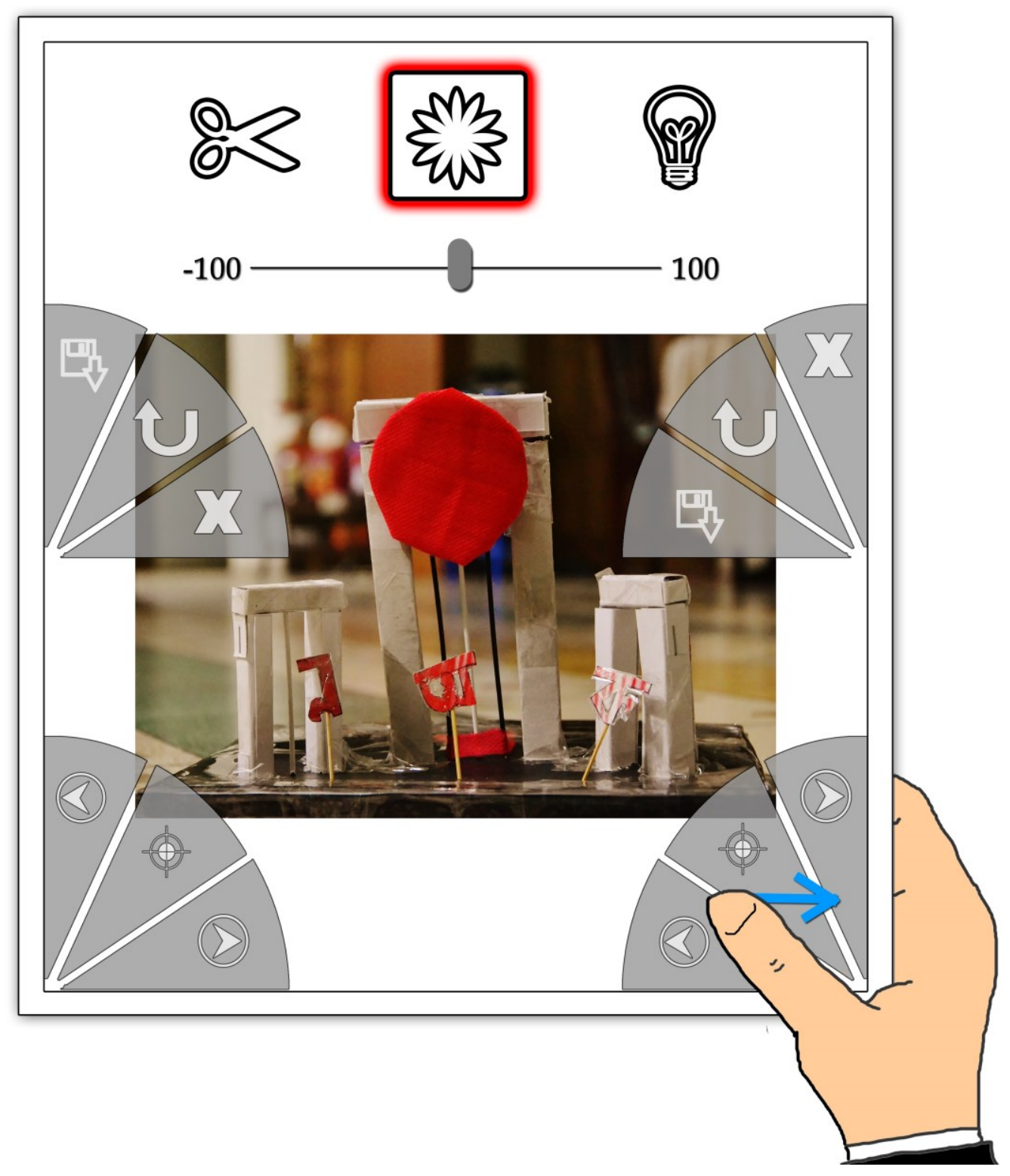

Figure 22: In the photo editing application, the user can increase the contrast of the image by swiping horizontally with the thumb (blue arrow). 
For either hand, tapping the button (i.e. tapping region) in the left takes user to the previous option (i.e. successive option in the left), and tapping the button in the right takes user to the next option (i.e. successive option in the right). This design choice leverages the contextual assignment of the tapping regions for intuitive user interaction.

We can also speed up this navigation by using horizontal swipes. For example, a horizontal swipe can move the selection by three options in the direction of the swipe.

The middle tapping region is for activating the selected photo editing option. Activation of a photo editing option enables the functionalities of that option and visualizes them on the display. For instance, if the user activates the 'contrast' option, a horizontal bar will inform the user visually about the current contrast level and its minimum and maximum level (Figure 22). The user can choose to increase or decrease the contrast using horizontal swipes.

The tapping regions on side center hold for both hands allow users to 'undo' the previous action, 'save' the edited image, and 'close' the current image. These tapping regions are labeled with appropriate icons to help user understand their functionalities.

\subsubsection{Bimanual Thumb Input in Photo Editing Application}

The thumb input in previous scenarios and in our experimental design were only unimanual. Though we have not investigated bimanual interaction using thumb input in our prototypes, we envision thumb input to be used for bimanual input as well.

Within the same photo editing application illustrated in Figure 22, Figure 23 illustrates the potential usage of bimanual touch input using the thumb of the holding hand in a flexible tablet. 


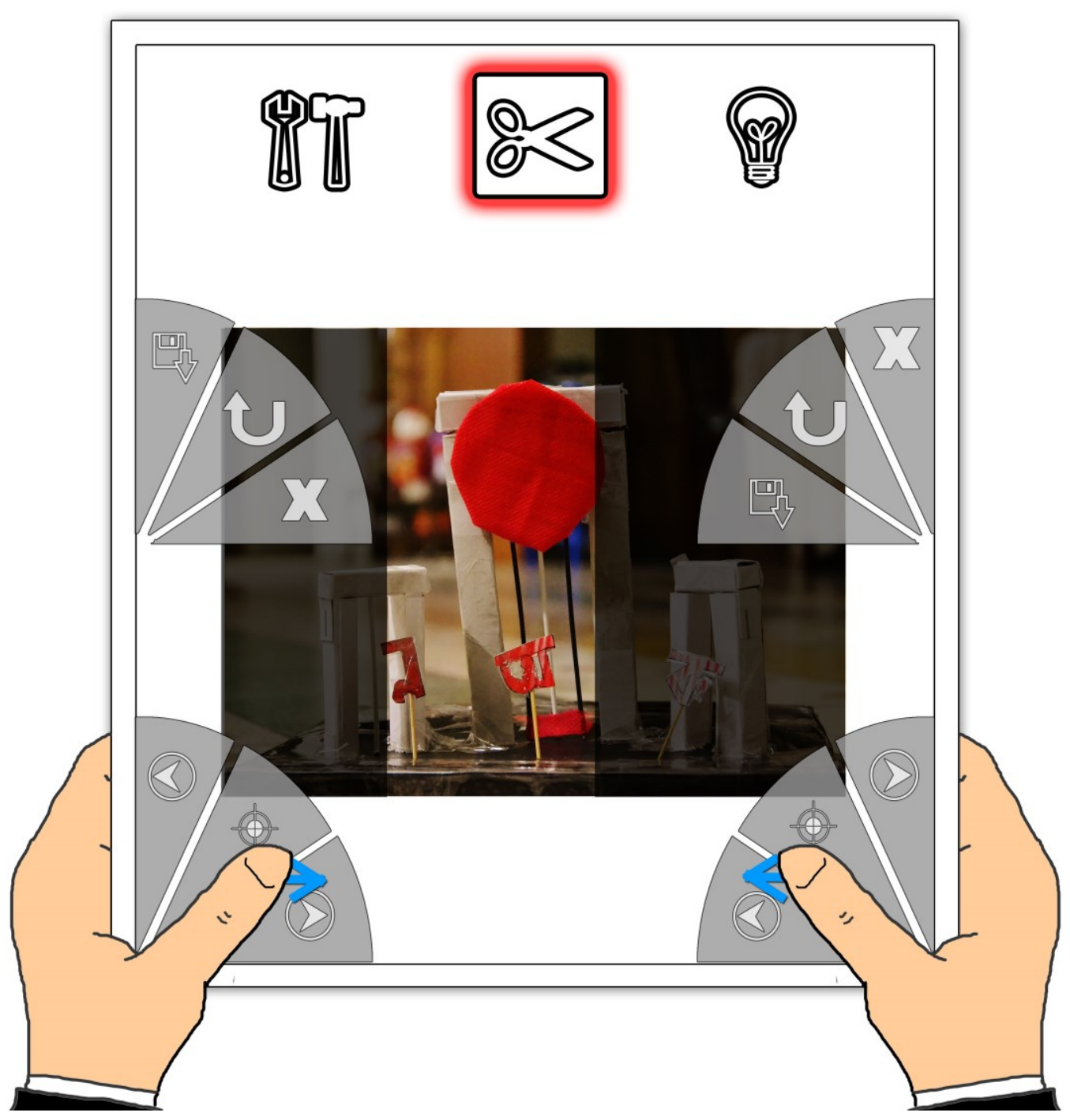

Figure 23: In the photo editing application, users can crop an image with bimanual thumb input using horizontal swipe (blue arrows)

'Cropping' is a common tool in photo editing application. The collection of photo editing options considered for this use case scenario includes 'cropping' option. After activating this option, users can crop the current image using horizontal and vertical swipes in the bottom corner hold using either or both hands. For instance, if users want to remove some portion of the image from left and right side, they can crop it separately for each side using horizontal swipe with one hand at a time, or they can do it simultaneously 
using both hands. Bimanual interaction is advantageous for superior user experience in many contexts $[18,24,32]$. This scenario visualizes the potential of thumb input to bring in the benefits of bimanual interactions to flexible tablets. 


\section{Chapter: Conclusion}

In this thesis, our primary goal was to explore the feasibility of thumb input on flexible displays. To achieve that goal, we evaluated two common touch interactions, tapping and swiping, between a flexible and a rigid prototype using the thumb of the holding hand. We found that participants had comparable performance for tapping and swiping in both flexible and rigid tablet prototypes. Participants also had similar preference for both rigidities for swiping interactions. Based on these findings, we recommended thumb input as a feasible touch input method on flexible handheld devices.

Our results also suggest that participants can handle both two and three tapping regions per hold with similar accuracy, and they can comfortably use three swipes: horizontal, vertical, and radial swipe. In addition, we observed that participants prefer to hold the device on the side or on the bottom corner. We also recommend using both hands for both tapping and swiping, which, we believe, can contribute in superior user experience by lessening the fatigue on one hand.

Based on our findings, we conclude that thumb input can bring the benefits of touch input in flexible displays. We believe the design guidelines we produced in this thesis to be particularly helpful for integrating thumb input on flexible handheld devices.

\subsection{Limitations}

We built our prototypes and designed the two studies with specific design goals and under some technical constrains. Although results from two studies support our design guidelines, we were bound by certain limitations, which we believe could be improved in several ways. 
Both of the experiments were conducted with a mock-up display by using projection instead of a real flexible display. The prototype was connected to a computer via a cable located on the top of the display. These two issues partially restricted participants' hand movements the experimental sessions, which might have contributed in generating hand fatigue. This constraint could be overcome by using a standalone flexible tablet with real flexible display.

We used the Zytronic flexible touch sensor [37], which detects touch input on the back surface of the sensor, in addition to detecting touch on the front surface. To restrict touch input on the back of the sensor, we used Styrofoam as an insulator, which increased the thickness of the prototype. A thinner prototype could be built by using a touch sensor that restricts touch input on the back surface.

We compared thumb input only between two rigidities: a flexible prototype and a rigid one. As future flexible tablets may come in different sizes and different levels of flexibility, we could compare the results with prototypes of different levels of flexibility and sizes.

All our participants were university students (mean age: 24.5 years). Otten et al. [22] suggested that age is a factor in thumb reach envelope, which in turn can have impact on our findings. This implies that our findings are not yet ready to be generalized for all age groups, though we believe that the impact of age will not be significant for our results as our evaluated interaction techniques are commonly used in handheld devices and are not particularly complex. However, it is worth evaluating our design guidelines with more diverse age groups to investigate the effect of age on our current findings. 
In addition, we evaluated both tapping and swiping interactions for simple tasks. However, everyday usage of tablets involves more practical tasks such as scrolling a web page or selecting items from a drop down menu. Therefore, it is worth testing our design guidelines with such practical tasks.

\subsection{Future Work}

In addition to the proposed solutions for the limitations above, there are many interesting studies that originate from the findings of this thesis. We identified three key research directions that can stem from this work. We briefly discuss them in the following subsections.

\subsubsection{Absolute Direct Touch vs. Augmented Touch-pad}

There have been multiple studies of thumb input on rigid handheld devices where the thumb input was used for augment touch-pad, i.e. that the thumb touch reachable zone represents the entire display size [12,36]. In both of our studies, we used thumb input for direct touch interaction. Therefore, it would be interesting to evaluate whether thumb input is best suited for absolute direct touch input [5], or to act as an augmented touch-pad in the domain of flexible handheld devices.

\subsubsection{Bimanual Unimodal Interaction with Thumb Input}

Bimanual unimodal interaction has been proved useful in rigid handheld devices $[18,24,32]$. Although we evaluated tapping and swiping with both dominant and nondominant hands, we designed both of our experiments for single-handed interaction. Hence, it would be worthy to investigate the effects of using both hands simultaneously for thumb input on flexible display. 
In addition, the effect of switching hands while using thumb input with one hand at a time on flexible displays can also be investigating.

\subsubsection{Bimanual Multimodal Interaction with Touch and Bend}

There has been a rapidly growing literature that combines bend and touch in order to achieve superior user experience in flexible handheld devices [3,14]. However, the literature on mixing bend and touch input on flexible displays within the same interaction cycle is still insufficient. We believe that thumb input will be particularly helpful to offer the benefits of bimanual multimodal interactions on flexible displays. The reason behind this is that the thumb input is executed by the hand holding the device, and the other hand is then freed to perform bend gestures, whether simultaneously or separately. Therefore, it would be interesting to evaluate bimanual interactions on flexible displays by using thumb input and bend gestures simultaneously.

With the advent of flexible handheld computing devices for mass consumption in near future, we believe that thumb input will play an important role to provide superior user experience. In that case, we are hopeful that the design guidelines provided in this thesis for integrating thumb input on flexible displays will be particularly useful. 


\title{
Appendices
}

\section{Appendix A Questionnaires}

\section{A.1 Pre-Test Questionnaires}

\section{Demographic Information}

Age:

Gender: $\square$ Male $\quad \square$ Female

Handedness: $\square$ Left $\quad \square$ Right $\square$ Ambidextrous

Indicate which of the following touch-sensitive devices you currently use (Mark all that apply):

\author{
$\square$ Smartphone \\ $\square$ Tablet \\ $\square$ eReader \\ $\square$ Music Player \\ $\square$ Tablet PC or Touch Screen computer
}

\section{Experiences with Touch Screen}

How often do you use a touch-sensitive device?

$\square$ Hourly

$\square$ Daily

$\square$ Several times a week

$\square$ Weekly

$\square$ Monthly 
Indicate how comfortable you are using touch-sensitive devices:

\begin{tabular}{|c|c|c|c|c|c|c|}
\hline Comfortable & 1 & 2 & 3 & 4 & 5 & Uncomfortable \\
\hline
\end{tabular}

Have you ever used a flexible (deformable) device?

$\square$ Yes $\quad \square$ No

If Yes, please explain: 


\section{A.2 Experiment 1 Questionnaires}

Flexible

Left Hand

3 Region

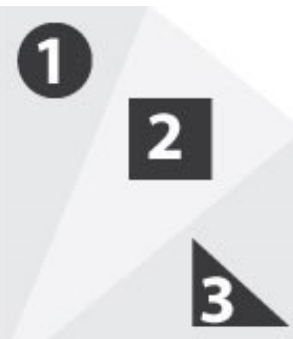

How difficult it was to use each Tapping Region?

\begin{tabular}{|c|c|c|c|c|c|c|c|c|}
\hline \multirow{3}{*}{ 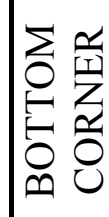 } & Tapping Region 1 & Easy & 1 & 2 & 3 & 4 & 5 & Difficult \\
\hline & Tapping Region 2 & Easy & 1 & 2 & 3 & 4 & 5 & Difficult \\
\hline & Tapping Region 3 & Easy & 1 & 2 & 3 & 4 & 5 & Difficult \\
\hline
\end{tabular}

\begin{tabular}{|c|c|c|c|c|c|c|c|c|}
\hline \multirow{3}{*}{ 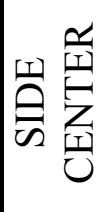 } & Tapping Region 1 & Easy & 1 & 2 & 3 & 4 & 5 & Difficult \\
\hline & Tapping Region 2 & Easy & 1 & 2 & 3 & 4 & 5 & Difficult \\
\hline & Tapping Region 3 & Easy & 1 & 2 & 3 & 4 & 5 & Difficult \\
\hline
\end{tabular}

\begin{tabular}{|c|c|c|c|c|c|c|c|c|}
\hline \multirow{3}{*}{ 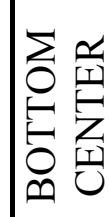 } & Tapping Region 1 & Easy & 1 & 2 & 3 & 4 & 5 & Difficult \\
\hline & Tapping Region 2 & Easy & 1 & 2 & 3 & 4 & 5 & Difficult \\
\hline & Tapping Region 3 & Easy & 1 & 2 & 3 & 4 & 5 & Difficult \\
\hline
\end{tabular}




\section{1 \\ 2

How difficult it was to use each Tapping Region?

\begin{tabular}{|c|c|c|c|c|c|c|c|c|}
\hline \multirow{3}{*}{ 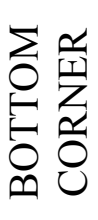 } & Tapping Region 1 & Easy & 1 & 2 & 3 & 4 & 5 & Difficult \\
\hline & Tapping Region 2 & Easy & 1 & 2 & 3 & 4 & 5 & Difficult \\
\hline & Tapping Region 3 & Easy & 1 & 2 & 3 & 4 & 5 & Difficult \\
\hline
\end{tabular}

\begin{tabular}{|c|c|c|c|c|c|c|c|c|}
\hline \multirow{3}{*}{ 拿点 } & Tapping Region 1 & Easy & 1 & 2 & 3 & 4 & 5 & Difficult \\
\hline & Tapping Region 2 & Easy & 1 & 2 & 3 & 4 & 5 & Difficult \\
\hline & Tapping Region 3 & Easy & 1 & 2 & 3 & 4 & 5 & Difficult \\
\hline
\end{tabular}

\begin{tabular}{|c|c|c|c|c|c|c|c|c|}
\hline \multirow{3}{*}{ 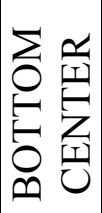 } & Tapping Region 1 & Easy & 1 & 2 & 3 & 4 & 5 & Difficult \\
\hline & Tapping Region 2 & Easy & 1 & 2 & 3 & 4 & 5 & Difficult \\
\hline & Tapping Region 3 & Easy & 1 & 2 & 3 & 4 & 5 & Difficult \\
\hline
\end{tabular}




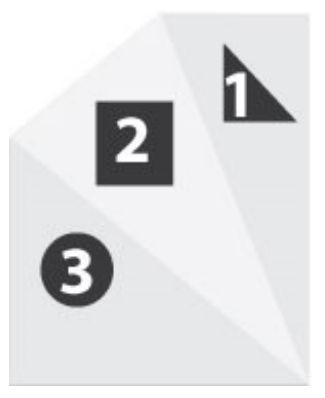

How difficult it was to use each Tapping Region?

\begin{tabular}{|c|c|c|c|c|c|c|c|c|}
\hline \multirow{3}{*}{ 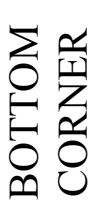 } & Tapping Region 1 & Easy & 1 & 2 & 3 & 4 & 5 & Difficult \\
\hline & Tapping Region 2 & Easy & 1 & 2 & 3 & 4 & 5 & Difficult \\
\hline & Tapping Region 3 & Easy & 1 & 2 & 3 & 4 & 5 & Difficult \\
\hline
\end{tabular}

\begin{tabular}{|c|c|c|c|c|c|c|c|c|}
\hline \multirow{3}{*}{ 㭊泀 } & Tapping Region 1 & Easy & 1 & 2 & 3 & 4 & 5 & Difficult \\
\hline & Tapping Region 2 & Easy & 1 & 2 & 3 & 4 & 5 & Difficult \\
\hline & Tapping Region 3 & Easy & 1 & 2 & 3 & 4 & 5 & Difficult \\
\hline
\end{tabular}

\begin{tabular}{|c|c|c|c|c|c|c|c|c|}
\hline \multirow{3}{*}{ 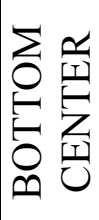 } & Tapping Region 1 & Easy & 1 & 2 & 3 & 4 & 5 & Difficult \\
\hline & Tapping Region 2 & Easy & 1 & 2 & 3 & 4 & 5 & Difficult \\
\hline & Tapping Region 3 & Easy & 1 & 2 & 3 & 4 & 5 & Difficult \\
\hline
\end{tabular}




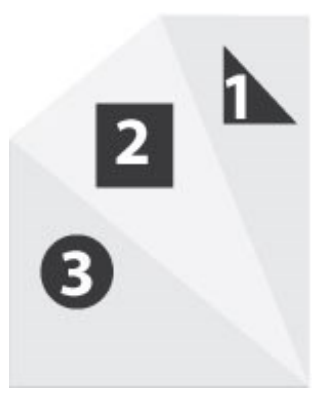

How difficult it was to use each Tapping Region?

\begin{tabular}{|c|c|c|c|c|c|c|c|c|}
\hline \multirow{3}{*}{ 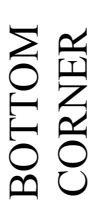 } & Tapping Region 1 & Easy & 1 & 2 & 3 & 4 & 5 & Difficult \\
\hline & Tapping Region 2 & Easy & 1 & 2 & 3 & 4 & 5 & Difficult \\
\hline & Tapping Region 3 & Easy & 1 & 2 & 3 & 4 & 5 & Difficult \\
\hline
\end{tabular}

\begin{tabular}{|c|c|c|c|c|c|c|c|c|}
\hline \multirow{3}{*}{ 㭊泀 } & Tapping Region 1 & Easy & 1 & 2 & 3 & 4 & 5 & Difficult \\
\hline & Tapping Region 2 & Easy & 1 & 2 & 3 & 4 & 5 & Difficult \\
\hline & Tapping Region 3 & Easy & 1 & 2 & 3 & 4 & 5 & Difficult \\
\hline
\end{tabular}

\begin{tabular}{|c|c|c|c|c|c|c|c|c|}
\hline \multirow{3}{*}{ 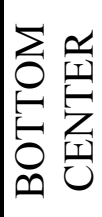 } & Tapping Region 1 & Easy & 1 & 2 & 3 & 4 & 5 & Difficult \\
\hline & Tapping Region 2 & Easy & 1 & 2 & 3 & 4 & 5 & Difficult \\
\hline & Tapping Region 3 & Easy & 1 & 2 & 3 & 4 & 5 & Difficult \\
\hline
\end{tabular}




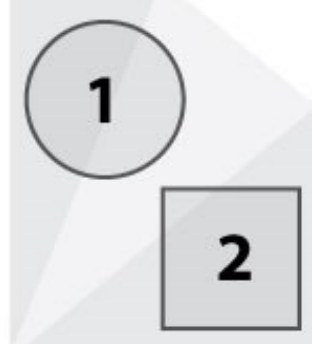

How difficult it was to use each Tapping Region?

\begin{tabular}{|c|c|c|c|c|c|c|c|c|}
\hline \multirow{2}{*}{ 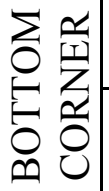 } & Tapping Region 1 & Easy & 1 & 2 & 3 & 4 & 5 & Difficult \\
\hline & Tapping Region 2 & Easy & 1 & 2 & 3 & 4 & 5 & Difficult \\
\hline
\end{tabular}

\begin{tabular}{|c|c|c|c|c|c|c|c|c|}
\hline & Tapping Region 1 & Easy & 1 & 2 & 3 & 4 & 5 & Difficult \\
\hline ن & Tapping Region 2 & Easy & 1 & 2 & 3 & 4 & 5 & Difficult \\
\hline
\end{tabular}

\begin{tabular}{|c|c|c|c|c|c|c|c|c|}
\hline \multirow{2}{*}{ 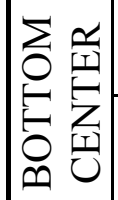 } & Tapping Region 1 & Easy & 1 & 2 & 3 & 4 & 5 & Difficult \\
\hline & Tapping Region 2 & Easy & 1 & 2 & 3 & 4 & 5 & Difficult \\
\hline
\end{tabular}




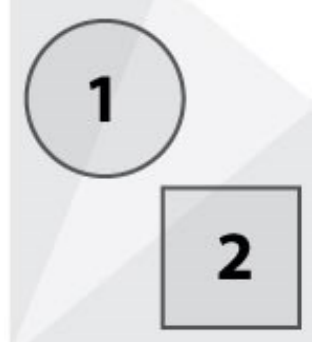

How difficult it was to use each Tapping Region?

\begin{tabular}{|c|c|c|c|c|c|c|c|c|}
\hline \multirow{2}{*}{ 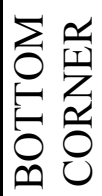 } & Tapping Region 1 & Easy & 1 & 2 & 3 & 4 & 5 & Difficult \\
\hline & Tapping Region 2 & Easy & 1 & 2 & 3 & 4 & 5 & Difficult \\
\hline
\end{tabular}

\begin{tabular}{|c|c|c|c|c|c|c|c|c|}
\hline \multirow{2}{*}{ 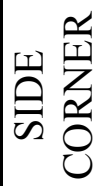 } & Tapping Region 1 & Easy & 1 & 2 & 3 & 4 & 5 & Difficult \\
\hline & Tapping Region 2 & Easy & 1 & 2 & 3 & 4 & 5 & Difficult \\
\hline
\end{tabular}

\begin{tabular}{|c|c|c|c|c|c|c|c|c|}
\hline$\sum_{0} \frac{\alpha}{\underline{I}}$ & Tapping Region 1 & Easy & 1 & 2 & 3 & 4 & 5 & Difficult \\
\hline 0 & Tapping Region 2 & Easy & 1 & 2 & 3 & 4 & 5 & Difficult \\
\hline
\end{tabular}




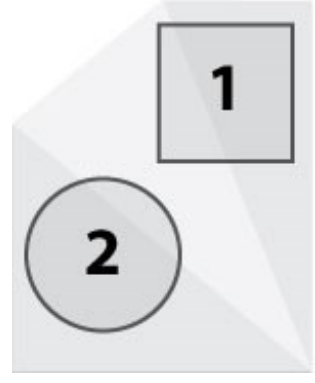

How difficult it was to use each Tapping Region?

\begin{tabular}{|c|c|c|c|c|c|c|c|c|}
\hline \multirow{2}{*}{$\mid \begin{array}{l}\sum_{0} \\
0 \\
0 \\
0 \\
0 \\
0 \\
0 \\
0 \\
0\end{array}$} & Tapping Region 1 & Easy & 1 & 2 & 3 & 4 & 5 & Difficult \\
\hline & Tapping Region 2 & Easy & 1 & 2 & 3 & 4 & 5 & Difficult \\
\hline
\end{tabular}

\begin{tabular}{|c|c|c|c|c|c|c|c|c|}
\hline & Tapping Region 1 & Easy & 1 & 2 & 3 & 4 & 5 & Difficult \\
\hline ن & Tapping Region 2 & Easy & 1 & 2 & 3 & 4 & 5 & Difficult \\
\hline
\end{tabular}

\begin{tabular}{|c|c|c|c|c|c|c|c|c|}
\hline \multirow{2}{*}{ 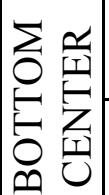 } & Tapping Region 1 & Easy & 1 & 2 & 3 & 4 & 5 & Difficult \\
\hline & Tapping Region 2 & Easy & 1 & 2 & 3 & 4 & 5 & Difficult \\
\hline
\end{tabular}




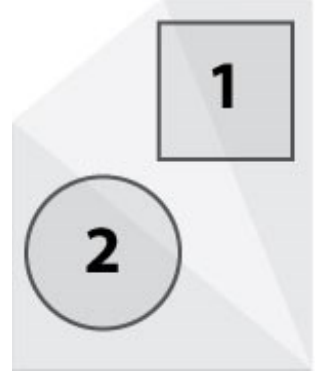

How difficult it was to use each Tapping Region?

\begin{tabular}{|c|c|c|c|c|c|c|c|c|}
\hline \multirow{2}{*}{ 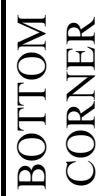 } & Tapping Region 1 & Easy & 1 & 2 & 3 & 4 & 5 & Difficult \\
\hline & Tapping Region 2 & Easy & 1 & 2 & 3 & 4 & 5 & Difficult \\
\hline
\end{tabular}

\begin{tabular}{|c|c|c|c|c|c|c|c|c|}
\hline \multirow{2}{*}{ 䎡舀 } & Tapping Region 1 & Easy & 1 & 2 & 3 & 4 & 5 & Difficult \\
\hline & Tapping Region 2 & Easy & 1 & 2 & 3 & 4 & 5 & Difficult \\
\hline
\end{tabular}

\begin{tabular}{|c|c|c|c|c|c|c|c|c|}
\hline \multirow{2}{*}{ 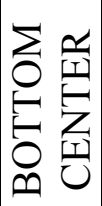 } & Tapping Region 1 & Easy & 1 & 2 & 3 & 4 & 5 & Difficult \\
\hline & Tapping Region 2 & Easy & 1 & 2 & 3 & 4 & 5 & Difficult \\
\hline
\end{tabular}




\section{A.3 Experiment 2 Questionnaires}

Flexible

Left Hand

For each Swipe Gesture, please indicate how difficult it was to perform the gesture:

\begin{tabular}{|c|c|c|c|c|c|c|c|c|c|}
\hline \multirow{7}{*}{ 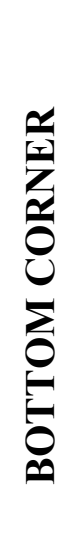 } & $\begin{array}{l}\text { Horizontal } \\
\text { Swipe }\end{array}$ & $\rightleftarrows \rightarrow$ & Easy & 1 & 2 & 3 & 4 & 5 & Difficult \\
\hline & & & & & & & & & \\
\hline & $\begin{array}{l}\text { Vertical } \\
\text { Swipe }\end{array}$ & & Easy & 1 & 2 & 3 & 4 & 5 & Difficult \\
\hline & & & & & & & & & \\
\hline & $\begin{array}{l}\text { Swipe } \\
\text { Swal }\end{array}$ & & Easy & 1 & 2 & 3 & 4 & 5 & Difficult \\
\hline & Radial & & & & & & & & \\
\hline & Swipe & & Easy & 1 & 2 & 3 & 4 & 5 & Difficult \\
\hline
\end{tabular}

\begin{tabular}{|c|c|c|c|c|c|c|c|c|c|}
\hline \multirow{6}{*}{ 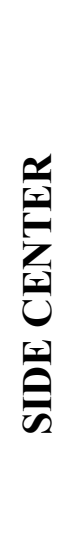 } & $\begin{array}{l}\text { Horizontal } \\
\text { Swipe }\end{array}$ & $\leftrightarrow \rightarrow$ & Easy & 1 & 2 & 3 & 4 & 5 & Difficult \\
\hline & & & & & & & & & \\
\hline & $\begin{array}{l}\text { Vertical } \\
\text { Swipe }\end{array}$ & & Easy & 1 & 2 & 3 & 4 & 5 & Difficult \\
\hline & $\begin{array}{l}\text { Diagonal } \\
\text { Swipe }\end{array}$ & & Easy & 1 & 2 & 3 & 4 & 5 & Difficult \\
\hline & $\begin{array}{l}\text { Radial } \\
\end{array}$ & & & & & & & & \\
\hline & Swipe & & Easy & 1 & 2 & 3 & 4 & 5 & Difficult \\
\hline
\end{tabular}

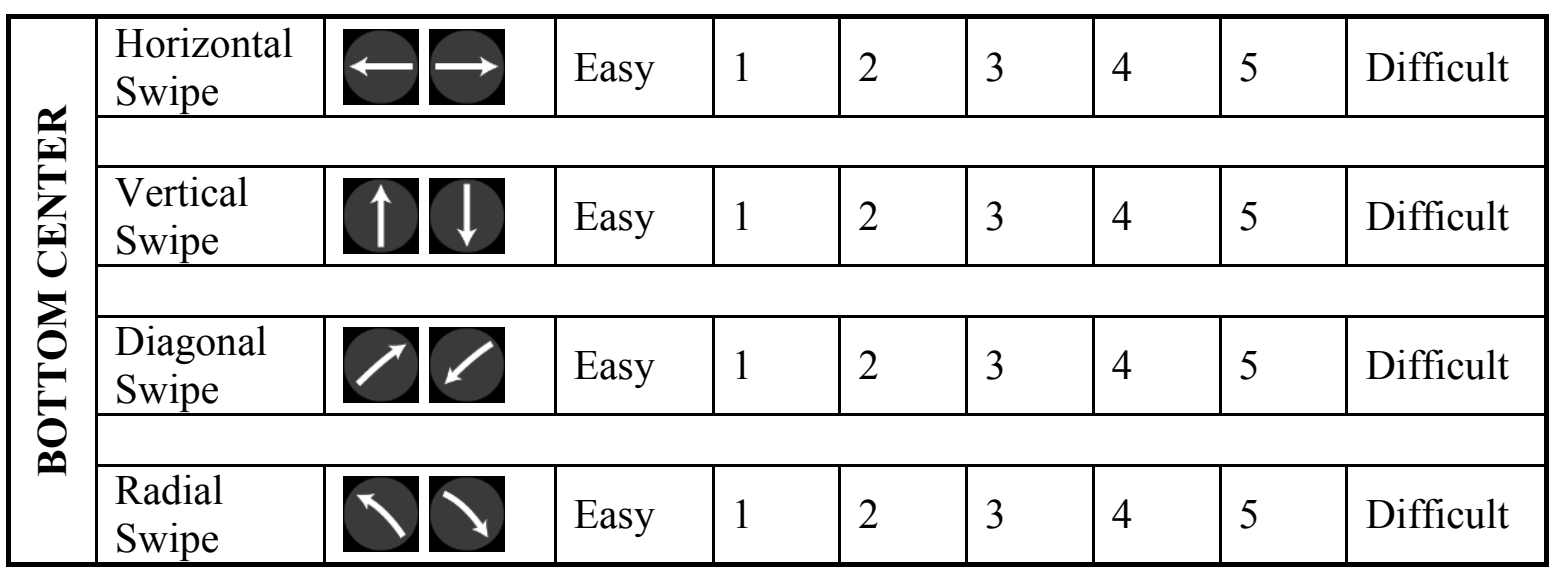


For each Swipe Gesture, please indicate how difficult it was to perform the gesture:

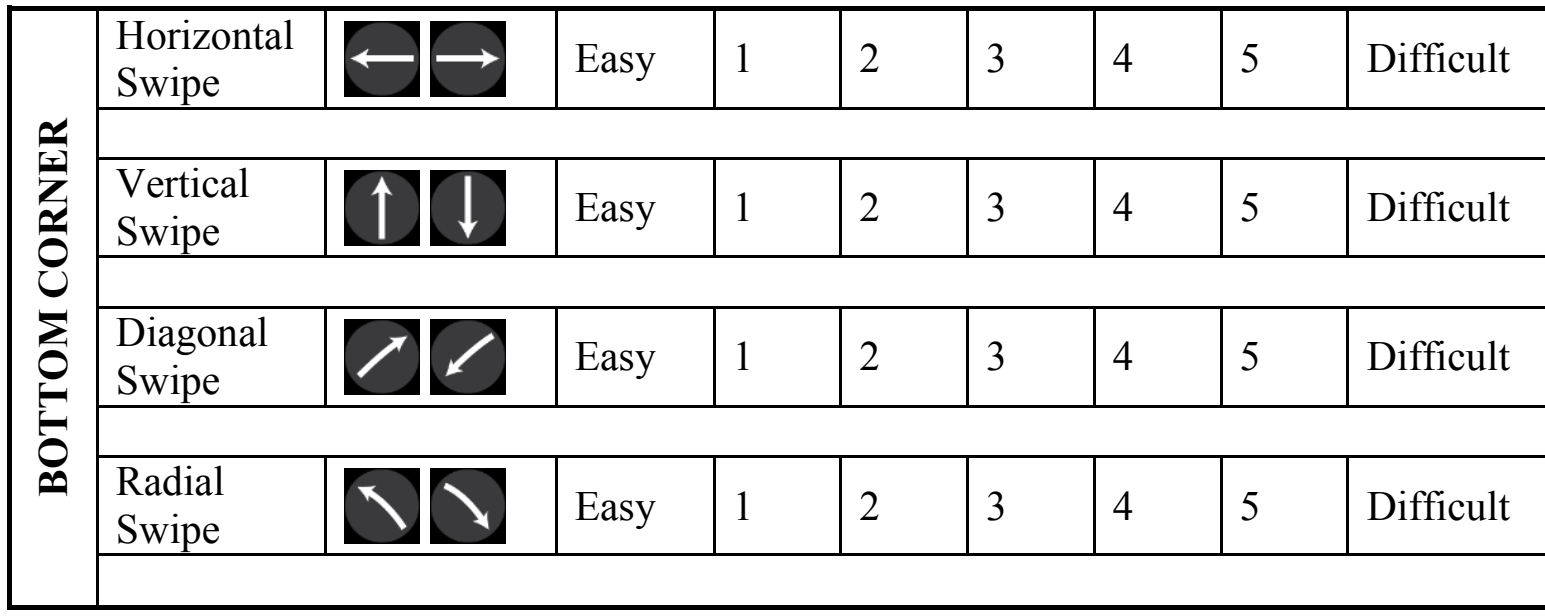

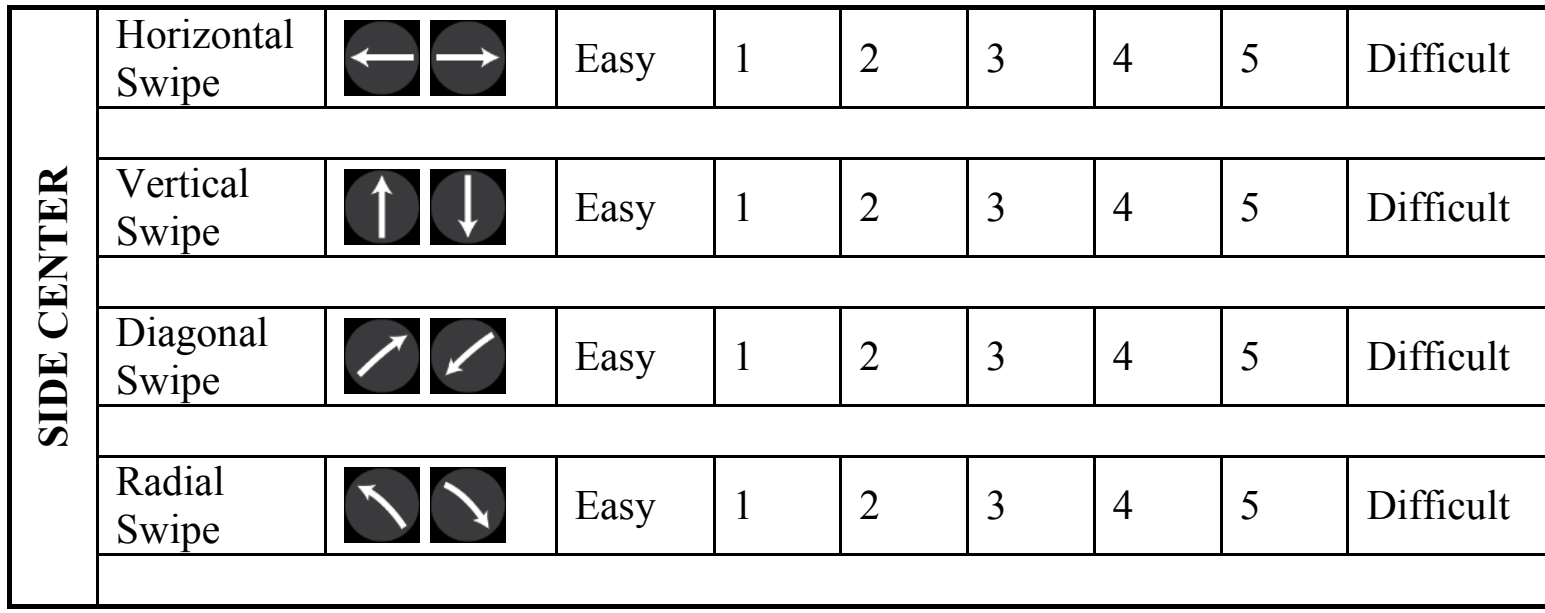

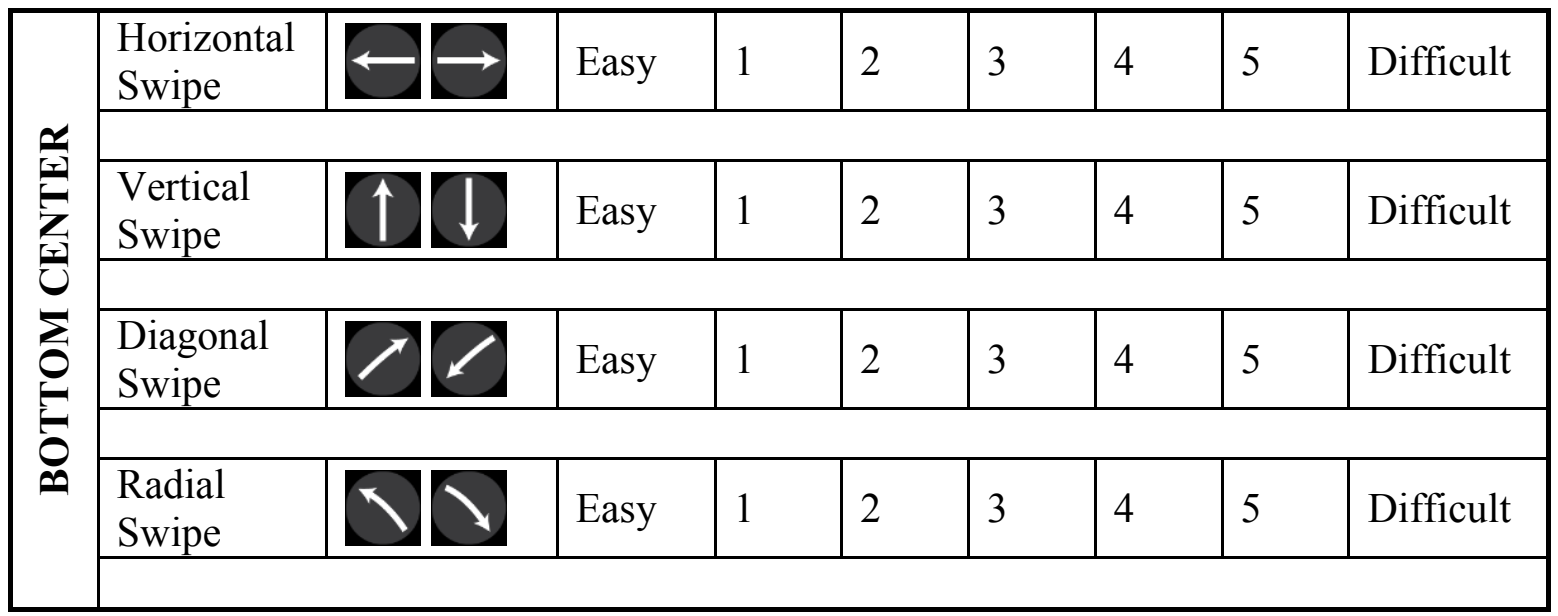


For each Swipe Gesture, please indicate how difficult it was to perform the gesture:

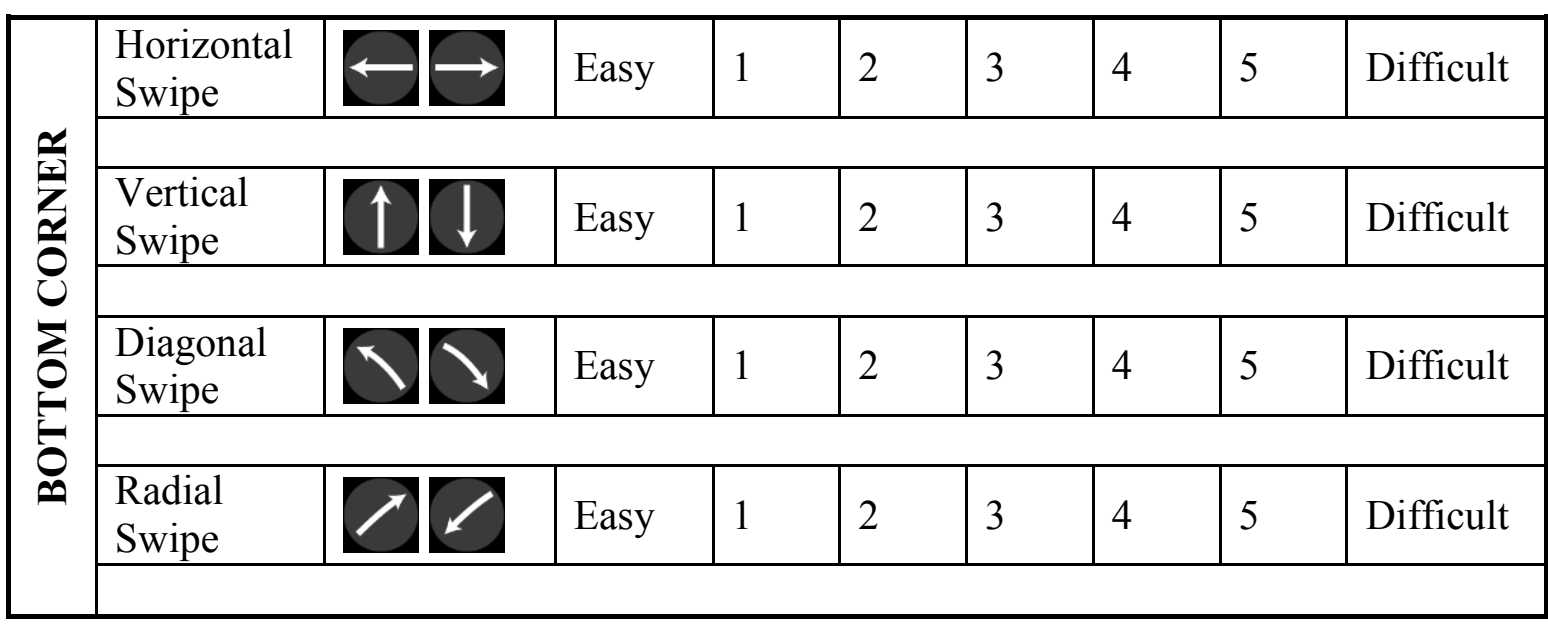

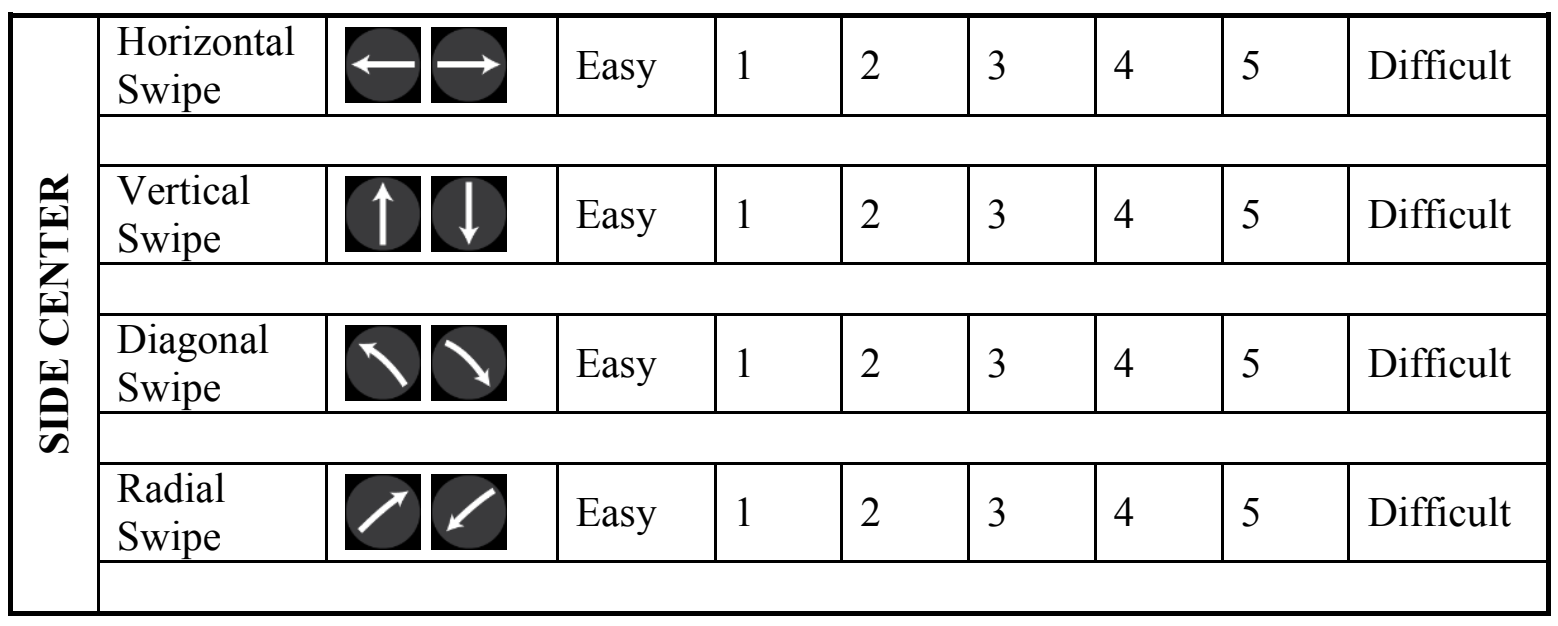

\begin{tabular}{|c|c|c|c|c|c|c|c|c|c|}
\hline \multirow{7}{*}{ 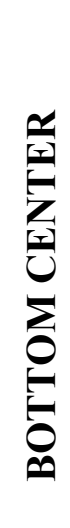 } & \begin{tabular}{|l} 
Horizontal \\
Swipe
\end{tabular} & $\leftrightarrow \rightarrow$ & Easy & 1 & 2 & 3 & 4 & 5 & Difficult \\
\hline & & & & & & & & & \\
\hline & $\begin{array}{l}\text { Vertical } \\
\text { Swipe } \\
\end{array}$ & & Easy & 1 & 2 & 3 & 4 & 5 & Difficult \\
\hline & \begin{tabular}{|l|} 
Diagonal \\
\end{tabular} & & & & & & & & \\
\hline & Swipe & & Easy & 1 & 2 & 3 & 4 & 5 & Difficult \\
\hline & & & & & & & & & \\
\hline & Swipe & & Easy & 1 & 2 & 3 & 4 & 5 & Difficult \\
\hline
\end{tabular}


For each Swipe Gesture, please indicate how difficult it was to perform the gesture:

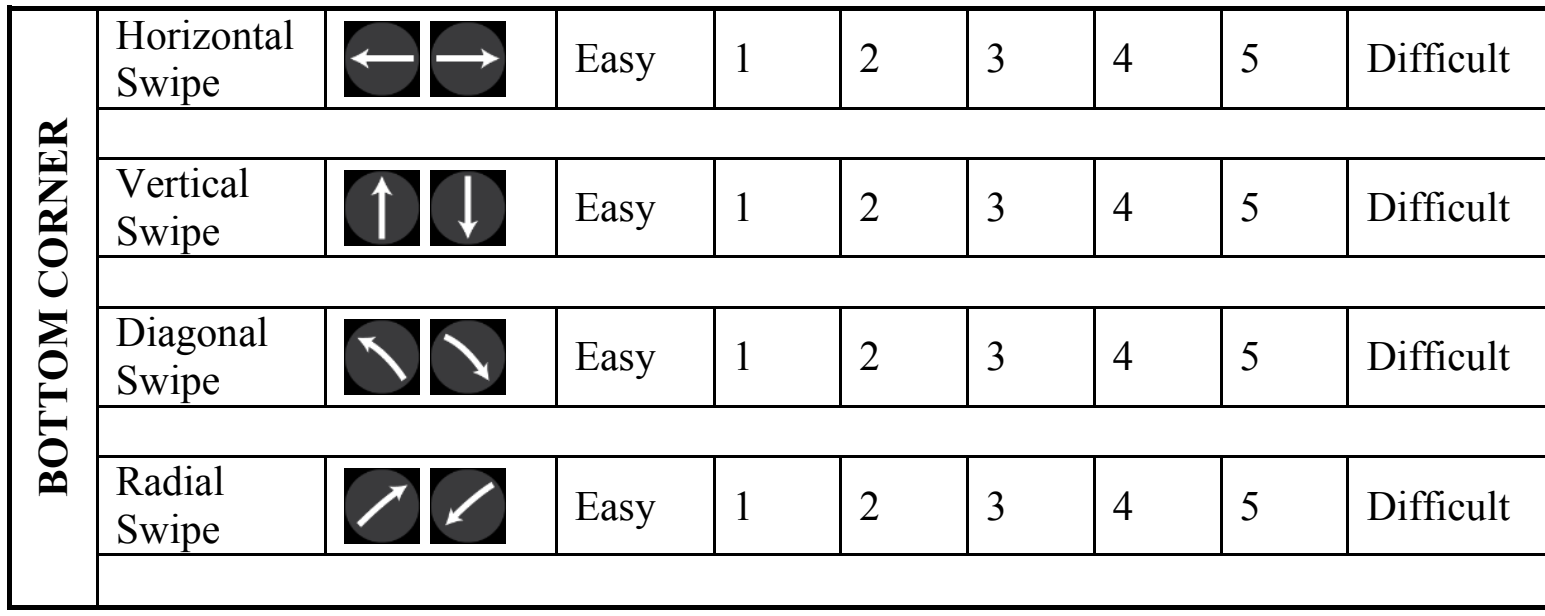

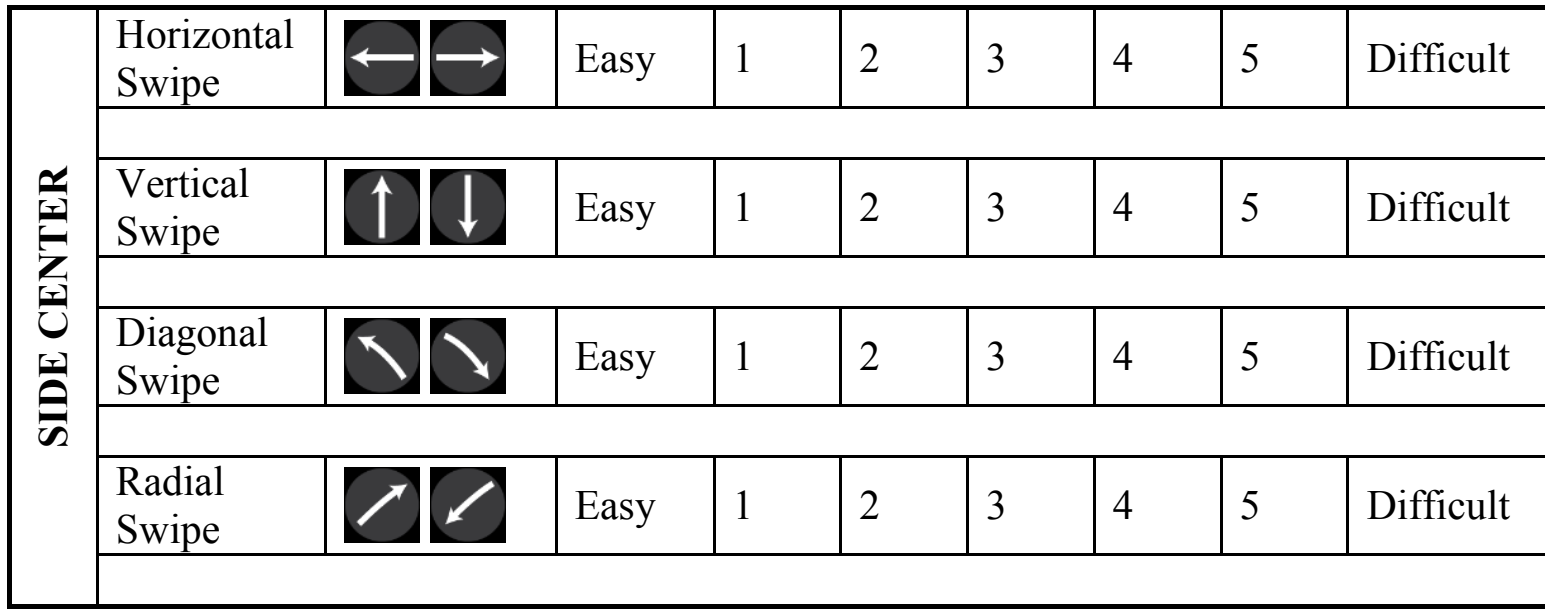

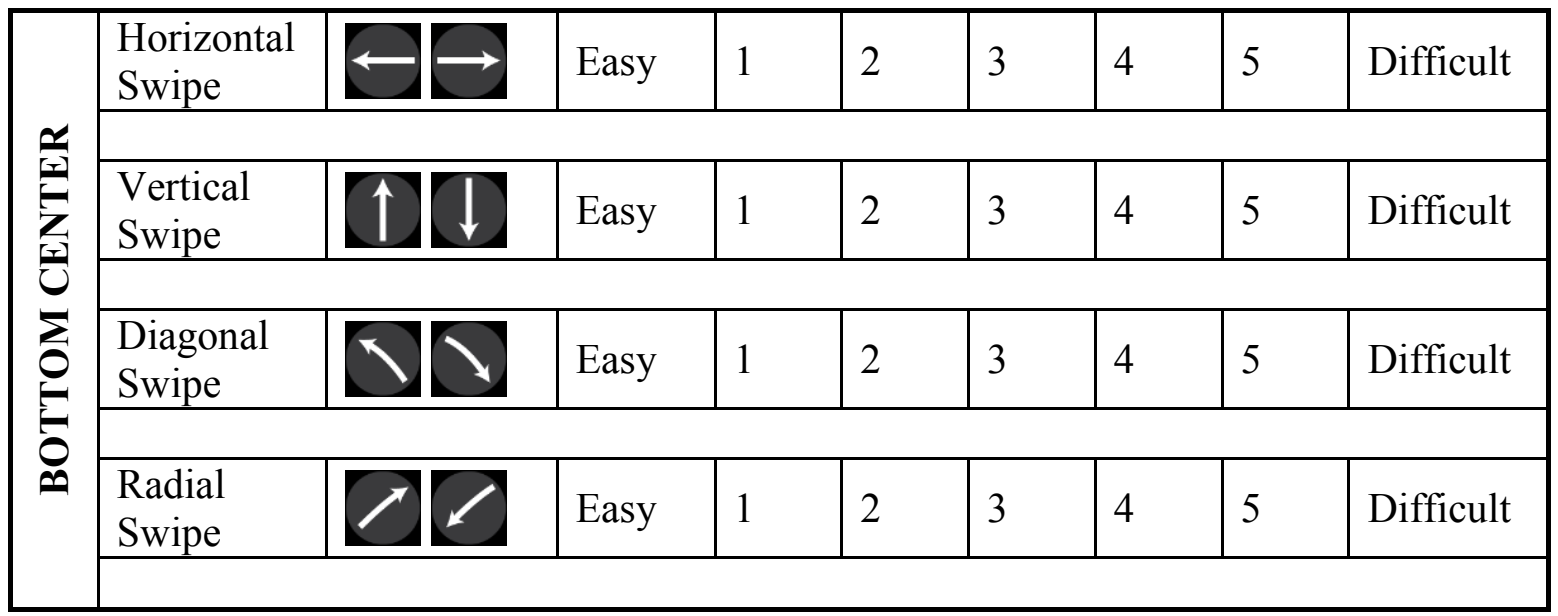




\section{A.4 Overall Post-Test Questionnaires}

- Overall, how difficult it was to perform the tapping gestures with left hand

\begin{tabular}{|l|l|l|l|l|l|l|}
\hline Easy & 1 & 2 & 3 & 4 & 5 & Difficult \\
\hline
\end{tabular}

- Overall, it was difficult to perform the tapping gestures with right hand

\begin{tabular}{|l|l|l|l|l|l|l|}
\hline Easy & 1 & 2 & 3 & 4 & 5 & Difficult \\
\hline
\end{tabular}

- Overall, it was difficult to perform the swipe gestures with left hand

\begin{tabular}{|l|l|l|l|l|l|l|}
\hline Easy & 1 & 2 & 3 & 4 & 5 & Difficult \\
\hline
\end{tabular}

- Overall, it was difficult to perform the swipe gestures with right hand

\begin{tabular}{|l|l|l|l|l|l|l|}
\hline Easy & 1 & 2 & 3 & 4 & 5 & Difficult \\
\hline
\end{tabular}

- Overall, how did you like performing each interaction style? Number ( 1 to 4$)$ them in order of your preference (1 being the most preferred $)$

Tapping with left hand

Tapping with right hand

Swipe with left hand

__ Swipe with right hand 
- Overall, how comfortable were you with each hold position while interacting with the device?

\begin{tabular}{|l|l|l|l|l|l|l|l|l|}
\hline \multirow{2}{*}{} & Bottom-Corner & Comfortable & 1 & 2 & 3 & 4 & 5 & Uncomfortable \\
\cline { 2 - 10 } & Side-Center & Comfortable & 1 & 2 & 3 & 4 & 5 & Uncomfortable \\
\cline { 2 - 10 } & Bottom-Center & Comfortable & 1 & 2 & 3 & 4 & 5 & Uncomfortable \\
\cline { 2 - 9 } & Bottom-Corner & Comfortable & 1 & 2 & 3 & 4 & 5 & Uncomfortable \\
\cline { 2 - 9 } & Side-Center & Comfortable & 1 & 2 & 3 & 4 & 5 & Uncomfortable \\
\cline { 2 - 9 } & Bottom-Center & Comfortable & 1 & 2 & 3 & 4 & 5 & Uncomfortable \\
\hline
\end{tabular}

- For Left hand:

Indicate your preference for the number of tapping regions per hold (select only one)

$\square 2$ Regions

$\square 3$ Regions

$\square$ No preference

$\rightarrow$ Why do you prefer this?

- For Right hand:

Indicate your preference for the number of tapping regions per hold (select only one)

$\square 2$ Regions

$\square 3$ Regions

$\square$ No preference

$\rightarrow$ Why do you prefer this? 
- For Tapping, which device did you prefer?

$\square$ Flexible one

$\square$ Rigid one

$\square$ No preference

$\rightarrow$ Why do you prefer this?

- For Swipe Gestures, which device did you prefer?

$\square$ Flexible one

$\square$ Rigid one

$\square$ No preference

$\rightarrow$ Why do you prefer this?

- During this study, how was your overall experience of using touch input in the flexible device?

- Do you have any additional comments? 


\section{Appendix B Regression Line Values}

\section{B.1 Swipe Regression Line Values for the Flexible Prototype}

\begin{tabular}{|c|c|c|c|c|c|}
\hline \multicolumn{6}{|c|}{ Flexible Prototype } \\
\hline Hand & Type of Swipe & Hold Position & Y-intercept & Slope & $\mathrm{R}^{2}$ \\
\hline \multirow{12}{*}{ 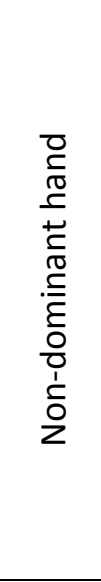 } & \multirow[t]{3}{*}{ Diagonal } & Side Center & 492.34 & 0.5476 & 0.329840 \\
\hline & & Bottom Corner & 59.08 & 0.9083 & 0.391930 \\
\hline & & Bottom Center & -37.22 & 0.3282 & 0.082435 \\
\hline & \multirow[t]{3}{*}{ Horizontal } & Side Center & 517.75 & -0.1554 & 0.118520 \\
\hline & & Bottom Corner & 109.54 & -0.0616 & 0.007590 \\
\hline & & Bottom Center & 170.19 & -0.2793 & 0.183810 \\
\hline & \multirow[t]{3}{*}{ Radial } & Side Center & 544.82 & -0.6155 & 0.296240 \\
\hline & & Bottom Corner & 160.49 & -0.7941 & 0.361820 \\
\hline & & Bottom Center & 209.72 & -0.4409 & 0.254360 \\
\hline & \multirow[t]{3}{*}{ Vertical } & Side Center & 397.74 & -4.4664 & 0.193910 \\
\hline & & Bottom Corner & 761.64 & -5.3009 & 0.514500 \\
\hline & & Bottom Center & 6010.12 & -16.5697 & 0.796860 \\
\hline \multirow{12}{*}{ 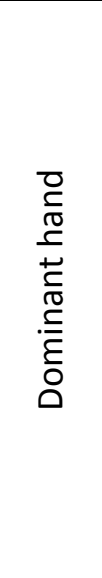 } & \multirow[t]{3}{*}{ Diagonal } & Side Center & 940.91 & -0.6658 & 0.358450 \\
\hline & & Bottom Corner & 606.87 & -0.7932 & 0.321270 \\
\hline & & Bottom Center & 306.48 & -0.5990 & 0.225610 \\
\hline & \multirow[t]{3}{*}{ Horizontal } & Side Center & 436.38 & 0.1145 & 0.024637 \\
\hline & & Bottom Corner & -65.75 & 0.2639 & 0.084991 \\
\hline & & Bottom Center & 80.07 & 0.0056 & 0.000090 \\
\hline & \multirow[t]{3}{*}{ Radial } & Side Center & -88.45 & 0.9491 & 0.437510 \\
\hline & & Bottom Corner & -640.87 & 1.1692 & 0.517020 \\
\hline & & Bottom Center & -44.51 & 0.3400 & 0.108660 \\
\hline & \multirow[t]{3}{*}{ Vertical } & Side Center & -5158.39 & 8.8719 & 0.834570 \\
\hline & & Bottom Corner & -3766.43 & 6.1983 & 0.182160 \\
\hline & & Bottom Center & 2791.62 & -8.2123 & 0.330430 \\
\hline
\end{tabular}




\section{B.2 Swipe Regression Line Values for the Rigid Prototype}

\begin{tabular}{|c|c|c|c|c|c|}
\hline \multicolumn{6}{|c|}{ Rigid Prototype } \\
\hline Hand & Type of Swipe & Hold Position & Y-intercept & Slope & $\mathbf{R}^{2}$ \\
\hline \multirow{12}{*}{ 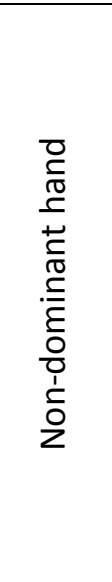 } & \multirow{3}{*}{ Diagonal } & Side Center & 467.28 & 0.7060 & 0.383660 \\
\hline & & Bottom Corner & 57.62 & 0.7975 & 0.359040 \\
\hline & & Bottom Center & 88.44 & 0.0550 & 0.0044501 \\
\hline & \multirow{3}{*}{ Horizontal } & Side Center & 519.54 & -0.1787 & 0.112720 \\
\hline & & Bottom Corner & 110.64 & -0.1608 & 0.052087 \\
\hline & & Bottom Center & 92.10 & -0.0801 & 0.015207 \\
\hline & \multirow{3}{*}{ Radial } & Side Center & 563.27 & -0.8067 & 0.521510 \\
\hline & & Bottom Corner & 149.87 & -0.7640 & 0.341740 \\
\hline & & Bottom Center & 240.68 & -0.4996 & 0.385500 \\
\hline & \multirow{3}{*}{ Vertical } & Side Center & 748.45 & -5.0513 & 0.456550 \\
\hline & & Bottom Corner & 413.93 & -4.7689 & 0.190580 \\
\hline & & Bottom Center & 3784.64 & -10.3284 & 0.309180 \\
\hline \multirow{12}{*}{ 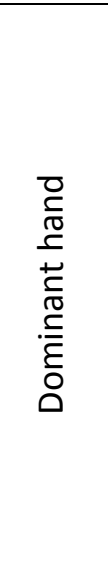 } & \multirow{3}{*}{ Diagonal } & Side Center & 911.09 & -0.6238 & 0.354710 \\
\hline & & Bottom Corner & 688.79 & -0.9184 & 0.41852 \\
\hline & & Bottom Center & 273.43 & -0.5359 & 0.305580 \\
\hline & \multirow{3}{*}{ Horizontal } & Side Center & 497.71 & 0.0095 & 0.000191 \\
\hline & & Bottom Corner & 239.90 & -0.2378 & 0.044643 \\
\hline & & Bottom Center & 13.45 & 0.1736 & 0.101880 \\
\hline & \multirow{3}{*}{ Radial } & Side Center & -68.36 & 0.4126 & 0.327770 \\
\hline & & Bottom Corner & -452.66 & 0.8882 & 0.363850 \\
\hline & & Bottom Center & 0.19 & 0.8028 & 0.412770 \\
\hline & \multirow{3}{*}{ Vertical } & Side Center & -3052.55 & 5.7200 & 0.46668 \\
\hline & & Bottom Corner & -4790.38 & 7.8446 & 0.543260 \\
\hline & & Bottom Center & 1898.37 & -6.2188 & 0.421930 \\
\hline
\end{tabular}




\section{References}

1. Bergstrom-Lehtovirta, J. and Oulasvirta, A. Modeling the functional area of the thumb on mobile touchscreen surfaces. Proc. CHI, (2014), 1991-2000.

2. Boring, S., Ledo, D., Chen, X.A., Marquardt, N., Tang, A., and Greenberg, S. The Fat Thumb: Using the Thumb's Contact Size for Single-Handed Mobile Interaction. Proc. MobileHCI, (2012), 39-48.

3. Burstyn, J., Banerjee, A., and Vertegaal, R. FlexView: An Evaluation of Depth Navigation on Deformable Mobile Devices. Proc. TEI, (2012), 193-200.

4. Dijkstra, R., Perez, C., and Vertegaal, R. Evaluating Effects of Structural Holds on Pointing and Dragging Performance with Flexible Displays. Proc. CHI, (2011), $1293-1302$.

5. Forlines, C., Vogel, D., and Balakrishnan, R. HybridPointing: Fluid Switching between Absolute and Relative Pointing with a Direct Input Device. Proc. UIST, (2006), 211-220.

6. Goubier, J., Devun, L., Mitton, D., and Lavaste, F. In vivo trapeziometacarpal joint kinematics using an optoelectronic system : a data basis on healthy subjects. Ninth Symposium On 3D Analysis Of Human Movement, (2006).

7. GRAND NCE. GRAND Researchers Unveil Revolutionary 'Paper Tablet' at 2013 CES. 2013. http://grand-nce.ca/newsandmedia/news-container/2013/grandresearchers-unveil-revolutionary-paper-tablet.

8. Henze, N., Rukzio, E., and Boll, S. 100,000,000 Taps: Analysis and Improvement of Touch Performance in the Large. Proc. MobileHCI, (2011), 133-142. 
9. Hinckley, K. and Song, H. Sensor Synaesthesia : Touch in Motion, and Motion in Touch. Proc. CHI, (2011), 801-810.

10. International Data Corporation. Press Release. 2013. http://www.idc.com/getdoc.jsp?containerId=prUS24314413.

11. Karlson, A., Bederson, B., and SanGiovanni, J. AppLens and LaunchTile: Two Designs for One-handed Thumb Use on Small Devices. Proc. CHI, (2011), 201210.

12. Karlson, A. and Bederson, B. ThumbSpace: Generalized One-handed Input for Touchscreen-based Mobile Devices. Proc. INTERACT, (2007), 324-338.

13. Karlson, A., Bederson, B., Contreras-Vidal, J.L. Understanding Single Handed Use of Handheld Devices. Handbook of Research on User Interface Design and Evaluation for Mobile Technology, Idea Group Reference (2006), 86-101.

14. Kildal, J., Lucero, A., and Boberg, M. Twisting Touch : Combining Deformation and Touch as Input within the Same Interaction Cycle on Handheld Devices. Proc. MobileHCI, (2013), 237-246.

15. Kim, S., Yu, J., and Lee, G. Interaction Techniques for Unreachable Objects on the Touchscreen. Proc. OzCHI, (2012), 295-298.

16. Lahey, B., Girouard, A., Burleson, W., and Vertegaal, R. PaperPhone: Understanding the Use of Bend Gestures in Mobile Devices with Flexible Electronic Paper Displays. Proc. CHI, (2011), 1303-1312.

17. Lee, S.-S., Kim, S., Jin, B., et al. How Users Manipulate Deformable Displays as Input Devices. Proc. CHI, (2010), 1647. 
18. Leganchuk, A., Zhai, S., and Buxton, W. Manual and Cognitive Benefits of Twohanded Input: An Experimental Study. Proc. TOCHI 5, 4 (1998), 326-359.

19. Malacria, S., Lecolinet, E., Guiard, Y. Clutch-Free Panning and Integrated PanZoom Control on Touch-Sensitive Surfaces : The CycloStar Approach. Proc. CHI, (2010), 2615-2624.

20. Micaux, M. and Beaudouin-lafon, M. SPad : A Bimanual Interaction Technique for Productivity Applications on Multi-Touch Tablets. Proc. CHI, (2014), 18791884.

21. Odell, D. and Chandrasekaran, V. Enabling Comfortable Thumb Interaction in Tablet Computers: A Windows 8 Case Study. Proc. HFES 56, 1 (2012), 19071911.

22. Otten, E.W., Karn, K.S., and Parsons, K.S. Defining Thumb Reach Envelopes for Handheld Devices. Human Factors: The Journal of the Human Factors and Ergonomics Society 55, 1 (2013), 48-60.

23. Oulasvirta, A., Reichel, A., Li, W., and Vertanen, K. Improving two-thumb text entry on touchscreen devices. Proc. CHI, (2013), 2765-2774.

24. Owen, R. and Kurtenbach, G. When It Gets More Difficult, Use Both Hands: Exploring Bimanual Curve Manipulation. Proc. Graphics Interface, (2005), 17 24.

25. Parhi, P., Karlson, A.K., and Bederson, B.B. Target Size Study for One-handed Thumb Use on Small Touchscreen Devices. Proc. MobileHCI, (2006), 203-210.

26. Park, Y.S., Han, S.H., Park, J., and Cho, Y. Touch Key Design for Target Selection on a Mobile Phone. Proc. MobileHCI, (2008), 423. 
27. Roudaut, A. and Huot, S. TapTap and MagStick : Improving One-Handed Target Acquisition on Small Touch-screens. Proc. AVI, (2008), 146-153.

28. Roudaut, A., Lecolinet, E., and Guiard, Y. MicroRolls: Expanding Touch-screen Input Vocabulary by Distinguishing Rolls vs. Slides of the Thumb. Proc. CHI, (2009), 927-936.

29. Schwesig, C., Poupyrev, I., and Mori, E. Gummi: A Bendable Computer. Proc. CHI, (2004), 263-270.

30. Tarun, A.P., Wang, P., Strohmeier, P.R., Girouard, A., Reilly, D., and Vertegaal, R. PaperTab: Tablets as Thin and Flexible as Paper. Proc. CHI EA, (2013).

31. Tilley, A. R., H.D.A. The Measure of Man and Woman: Human Factors in Design. Wiley, 2002.

32. Wagner, J., Huot, S., and Mackay, W. Bitouch and Bipad: Designing Bimanual Interaction for Hand-held Tablets. Proc. CHI 2012, May (2012), 2317-2326.

33. Warren, K., Lo, J., Vadgama, V., and Girouard, A. Bending the Rules: Bend Gesture Classification for Flexible Displays. Proc. CHI, (2013).

34. Watanabe, J., Mochizuki, A., and Horry, Y. Bookisheet: Bendable Device for Browsing Content Using the Metaphor of Leafing Through the Pages. Proc. UbiComp, (2008), 360-369.

35. Wightman, D., Ginn, T., and Vertegaal, R. BendFlip : Examining Input Techniques for Electronic Book Readers with Flexible Form Factors. Ifip International Federation For Information Processing 6948, (2011), 117-133. 
36. Yu, N.-H., Huang, D.-Y., Hsu, J.-J., and Hung, Y.-P. Rapid Selection of Hard-toAccess Targets by Thumb on Mobile Touch-Screens. Proc. MobileHCI, (2013), 400.

37. Zytronic Flexible Touch Sensor. http://www.zytronic.co.uk. 